\title{
Integrated Micro/Nanoengineered Functional Biomaterials for Cell Mechanics and Mechanobiology: A Materials Perspective
}

\author{
Yue Shao and Jianping Fu*
}

The rapid development of micro/nanoengineered functional biomaterials in the last two decades has empowered materials scientists and bioengineers to precisely control different aspects of the in vitro cell microenvironment. Following a philosophy of reductionism, many studies using synthetic functional biomaterials have revealed instructive roles of individual extracellular biophysical and biochemical cues in regulating cellular behaviors. Development of integrated micro/nanoengineered functional biomaterials to study complex and emergent biological phenomena has also thrived rapidly in recent years, revealing adaptive and integrated cellular behaviors closely relevant to human physiological and pathological conditions. Working at the interface between materials science and engineering, biology, and medicine, we are now at the beginning of a great exploration using micro/ nanoengineered functional biomaterials for both fundamental biology study and clinical and biomedical applications such as regenerative medicine and drug screening. In this review, an overview of state of the art micro/nanoengineered functional biomaterials that can control precisely individual aspects of cell-microenvironment interactions is presented and they are highlighted as well-controlled platforms for mechanistic studies of mechano-sensitive and -responsive cellular behaviors and integrative biology research. The recent exciting trend where micro/nanoengineered biomaterials are integrated into miniaturized biological and biomimetic systems for dynamic multiparametric microenvironmental control of emergent and integrated cellular behaviors is also discussed. The impact of integrated micro/nanoengineered functional biomaterials for future in vitro studies of regenerative medicine, cell biology, as well as human development and disease models are discussed.

\section{Introduction}

Cell populations within the human body are established, maintained and regulated within the adjacent microenvironment,

\footnotetext{
Y. Shao, Prof. J. Fu

Integrated Biosystems and Biomechanics Laboratory

Department of Mechanical Engineering

University of Michigan, Ann Arbor, 48109, USA

E-mail: jpfu@umich.edu

Prof. J. Fu

Department of Biomedical Engineering

University of Michigan

Ann Arbor, 48109, USA
}

DOI: 10.1002/adma.201304431 which actively signals to cells to regulate their fate and function. The microenvironmental factors, including cell-cell interactions, soluble factors such as oxygen tension and growth factors, and adhesive and biophysical interactions between cells and extracellular matrix (ECM), are all important for regulation of cellular behaviors. Cells and the surrounding microenvironment can also dynamically influence each other during normal development, tissue homeostasis and repair, and progression of diseases through their reciprocal biochemical and biophysical interactions..$^{[1-6]}$ Thus, a detailed apprehension and understanding of cell-microenvironment interactions is critical for both advancing basic biology knowledge and improving human health through regenerative medicine, developing in vitro human disease models and other therapeutic and diagnostic research.

A critical component for studying cell-microenvironment interactions is to create, characterize and manipulate dynamic microenvironmental cues in vitro down to a cellular (micrometer) and subcellular (nanometer) length scale. Over the last two decades, different micro/nanoengineering tools and synthesis methods for functional biomaterials have been successfully developed and applied for biological and biomedical research, establishing a rich toolbox of diverse micro/nanoengineered functional biomaterials for dynamic generation, modulation, stimulation of various extracellular biochemical and biophysical signals at a subcellular resolution to in vitro cultured cells. ${ }^{[7-12]}$ These micro/nanoengineered functional biomaterials have particularly helped reveal independent effects of individual biophysical signals of cell microenvironment, such as cell shape and geometry, ECM rigidity and topography, extracellular forces, and spatial organization of adhesive proteins, in regulating cellular behaviors such as cell migration, proliferation and survival, and differentiation. ${ }^{[13-23]}$ The rapid development of micro/nanoengineered functional biomaterials coupled with high-throughput screening tools and the concomitant discoveries of cellular mechano-sensitive and -responsive behaviors have culminated in recent excitements in mechanobiology, ${ }^{[9,20,24,25]}$ regenerative 
medicine, ${ }^{[14,26,27]}$ physical oncology, ${ }^{[17,18]}$ and cellular heterogeneity at the single-cell level. ${ }^{[28-32]}$

So far, many micro/nanoengineered functional biomaterials have been demonstrated useful for studying how individual biophysical signals of cell microenvironment can regulate cellular behaviors in vitro. However, to fully understand the functional role of cell-microenvironment interactions in regulating cell function, it is important to take into account the threedimensional (3D), multiparametric nature of in vivo cell microenvironment. ${ }^{[33-37]}$ The recent exciting trend of developing integrated miniaturized biological and biomimetic systems for dynamic multiparametric microenvironmental control to regulate emergent and integrated cellular behaviors has started to illustrate the impact of integrated micro/nanoengineered functional biomaterials for future research on regenerative medicine, cell biology, human normal development and diseases, as well as drug development.

In this review, we first present an overview of state of the art micro/nanoengineered functional biomaterials developed in the last two decades, which can be utilized to control and modulate various biophysical aspects of local cell microenvironment in vitro. In addition to their technical improvements, these micro/nanoengineered functional biomaterials have also been utilized for studying mechano-sensitive and -responsive cellular behaviors and underlying mechanotransduction mechanisms. We will then focus on reviewing the recent progress in integrating micro/nanoengineered functional biomaterials to develop miniaturized biological and biomimetic systems for dynamic multiparametric microenvironmental control of emergent and integrated cellular behaviors. We will conclude with remarks and future outlook. There are other recent informative reviews published elsewhere that provide detailed discussions specifically on micro/nanofabrication techniques for biomedical engineering, ${ }^{[8,38-43]}$ synthetic biomaterials as instructive extracellular microenvironments for tissue engineering and regenerative medicine, ${ }^{[26,44-47]}$ engineering 3D microenvironments for studying tissue physiology in vitro, ${ }^{33,34,39,48]}$ and mechanotransduction mechanisms in development and diseases. ${ }^{[2-5,18,20,24,49-53]}$ Readers interested in discussions in these specific topics are referred to these excellent reviews.

\section{Toolbox of Micro/Nanoengineered Functional Biomaterials}

In this section, we will present a review of state of the art micro/nanoengineered functional biomaterials developed in the last two decades that have allowed researchers to probe and characterize cell-microenvironment interactions as well as to obtain insights on mechano-sensitive and -responsive cellular properties in response to individual extracellular biophysical cues, including cell shape and geometry, ECM rigidity and topography, extracellular forces, and spatial organization of adhesive proteins. A detailed discussion of the purposes, developments, functional characteristics, variations and applications of different micro/nanoengineered functional biomaterials will be presented, to provide a foundation and guidance for future works in related research fields. We will discuss in detail the principles behind individual functional biomaterials

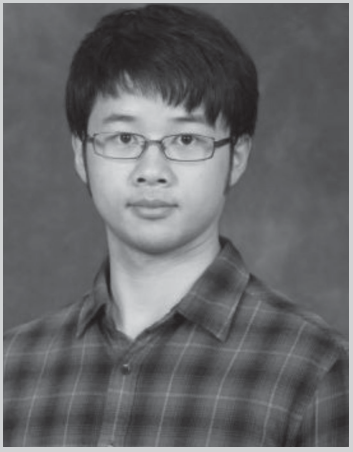

Yue Shao is a PhD candidate in the Department of Mechanical Engineering at the University of Michigan, Ann Arbor. He received his B.E. and M.E. degrees from Tsinghua University, China, in 2008 and 2011 , respectively. His current research interests include biomechanics of cellular mechanosensing and mechanotransduction of extracellular physical cues, stem cell mechanobiology, and micro/nanoengineering ex vivo cell microenvironment for regenerative medicine.

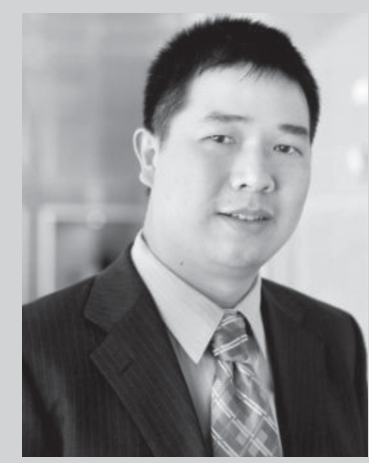

Dr. Jianping Fu has been an assistant professor of Mechanical and Biomedical Engineering at the University of Michigan, Ann Arbor since 2009. Dr. Fu received his Ph. D. degree from the Massachusetts Institute of Technology in 2007. He was an American Heart Association Postdoctoral Fellow at the University of Pennsylvania from 2007 to 2009. Dr. Fu's research focuses on mechanobiology, stem cell biology, and applying microfabrication technology to elucidate biosystems at molecular and cellular levels.

and technologies and explain critical steps or technical information associated with them. We will summarize and discuss their applications in studying mechano-sensitive and -responsive cellular properties.

\subsection{Micro/Nanoengineered Synthetic Substrates with Tunable Properties}

\subsubsection{Engineering Micro/Nanoscale Surface Topography}

In vivo, cells reside in the ECM with complex 3D architectures ranging from highly discrete and porous fibrous networks to continuous basement membranes, representing a vast topographical diversity of the local cell microenvironment. The characteristic size dimension of topographical features at the cell-ECM interface can range from tens (such as fibrillar collagens in the ECM of connective tissues) to a few nanometers (such as fibronectin fibrils). Interestingly, cell adhesion machineries (or the so called focal adhesions, FAs), which are mediated by dynamic clustering of transmembrane protein integrins, are also of a broad size range from about $10 \mathrm{~nm}$ to $10 \mu \mathrm{m}$, suggesting adherent cells may sense and respond to 
micro/nanoscale ECM topographical cues through cell-ECM adhesive interactions and downstream adhesion-mediated signaling. Using intravital microscopy, for example, it was observed that tumor cell migrated preferentially along ECM protein filaments during invasion in vivo, suggesting a close connection between cell adhesion, migration and ECM topography. ${ }^{[54]}$ Indeed, micro/nanoscale topography, structure, and architecture of fibrous ECM are important biophysical signals that can regulate cell adhesion and intracellular actin cytoskeleton organization and thus many cellular behaviors such as gene expression, proliferation, migration, and differentiation.

To understand how adherent cells sense and respond to ECM topography, various in vitro biomaterials with micro/ nanoscale surface topographic features have been developed and applied to perturb cell-ECM interfaces and regulate cell morphology and function. Synthetic substrates with micro/ nanoscale surface topographic features have helped reveal different 'topography-sensitive' cell phenotypes, which, in turn, illustrates a novel aspect of mechano-sensitive and -responsive cellular properties and provide foundations for innovative functional biomaterials incorporating unique topographic features to elicit desired cellular behaviors. In the following sessions, we will discuss different synthetic surfaces with regular topography, topography in a short-range order, or completely randomly distributed topography and their fabrication methods.

Micro/Nanoscale Topography in Regular Patterns. Different micromachining and microfabrication technologies have provided a vast array of tools to generate micro/nanoscale topography in regular patterns on synthetic surfaces. Among them, soft lithography, developed first by the Whitesides group at the Harvard University in late 1990 s, is one of the most popular methods. ${ }^{[8,38,55]}$ In general, soft lithography involves three separate and sequential steps - fabrication of masters using photolithography and micro/nanofabrication technologies, generating stamps from masters using replica molding and related molding techniques, and transfer of topographical features from stamps to synthetic surfaces (Figure 1a-d). ${ }^{[38]}$ Here, we briefly review critical steps in soft lithography.

Micromachining and microfabrication, which originates from the semiconductor industry and the micro-electromechanical systems (MEMS) field, apply photolithography and different dry and wet etching (such as reactive-ion etching, or RIE) and deposition techniques to fabricate micro/nanoscale structures on III-V semiconductor (including Silicon) wafers and glass and quartz substrates that are routinely used as masters in soft lithography. The high spatial resolution (down to $\sim 0.3 \mu \mathrm{m})$ and high positional and overlay accuracy $(<50 \mathrm{~nm})$ of the photolithography process allow masters to have welldefined prescribed regular structural patterns. The spatial resolution of photolithography can be improved down to sub-10 nm using advanced lithography tools such as electron beam lithography (EBL), X-ray lithography, and ion beam lithography (IBL). Although highly precise, EBL and IBL have a significantly lower throughput as patterns in both methods are generated by serial exposure of interconnected dots, and thus it takes a long time to generate large patterns using EBL and IBL. X-ray lithography is an expensive technology, limiting its routine access.

There are other useful approaches for fabrication of masters with micro/nanoscale topography in regular patterns. ${ }^{[56]}$

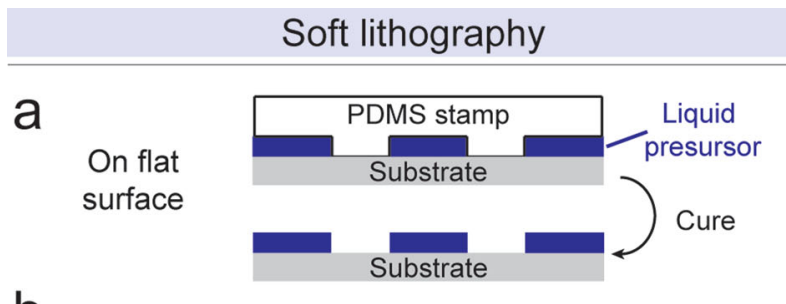

O

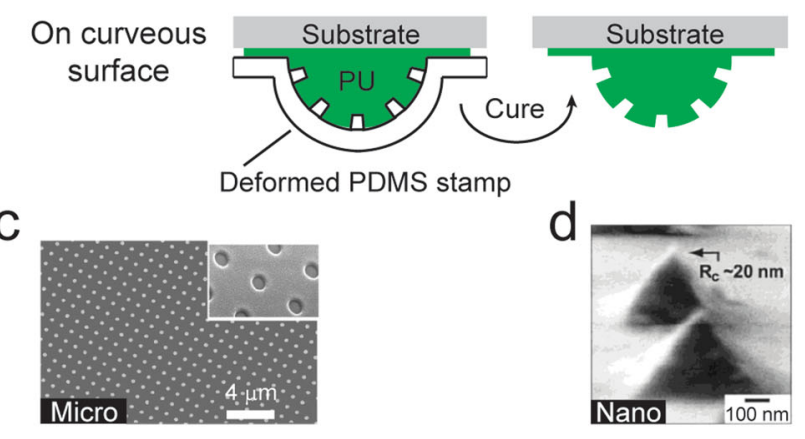

Liquid-bridge-mediated nanotransfer moulding

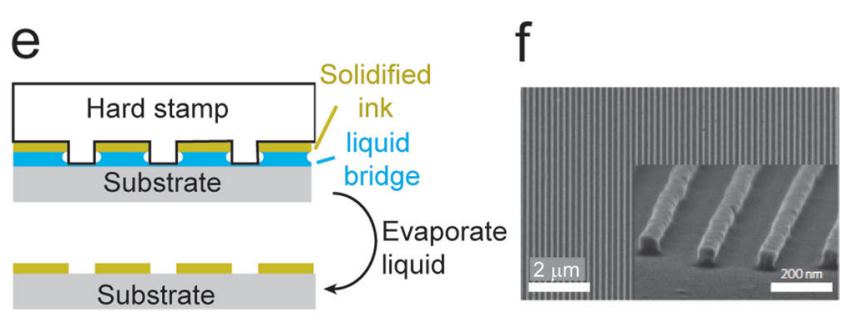

Figure 1. Fabricating micro/nanotopography in regular patterns. (a) Schematic of fabricating micro/nano-scale structures on a flat surface using soft lithography. ${ }^{[38]}$ A PDMS stamp was used to mold the liquid precursor and was peeled off after the polymer was cured. (b) Schematic of fabricating micro/nano-scale features on curvilinear surfaces using soft lithography. ${ }^{[61]}$ A pre-deformed PDMS stamp was used for generating the curvilinear surface, on which secondary micro/nano-scale features were molded. (c) SEM image of microtopography fabricated by soft lithography. Reproduced with permission. ${ }^{[38]}$ Copyright 2009, Nature Publishing Group. (d) SEM image of nano-scale surface structures fabricated by soft lithography, during which an $h$-PDMS/PDMS composite stamp was used to facilitate high geometrical fidelity. Reproduced with permission. ${ }^{[60]}$ Copyright 2002, American Chemical Society. (e) Schematic of the liquid-bridge-mediated nanotransfer moulding (LB-nTM) ${ }^{[63]} \mathrm{A}$ hard PDMS stamp loaded with nano-patterned foreign materials was put into contact with a thin liquid layer on the substrate. When evaporating the liquid, the meniscus facilitated the transfer of nano-molded materials onto the substrate. ( $f$ ) SEM image of an array of zinc-tin oxide (ZTO) nanowires transferred onto silicon substrate using LB-nTM. Reproduced with permission. ${ }^{[63]}$ Copyright 2010, Nature Publishing Group.

Among them, block copolymer lithography, a process where block copolymer self-assembly is integrated with conventional lithographic patterning, is emerging as a promising technology. ${ }^{[56]}$ The ability of block copolymers to self-assemble into ordered micro/nanoscale domains allows for simple, low-cost patterning into underlying substrates. Since its initial conception, block copolymer lithography has been demonstrated using 
a variety of block copolymers, with research primarily focusing on all-organic diblock copolymers. The most common example is polystyrene-block-poly(methyl methacrylate) (PS-b-PMMA) where long-range ordering of micro/nanoscale domains has allowed applications on a commercial scale. Using PS/PMMA copolymers, for example, Morarui and colleagues developed a spontaneous pattern formation method by applying an electric field to PS/PMMA double films. ${ }^{[57]}$ It should be noted that spontaneously formed patterns in block copolymer lithography are not as diverse as those achievable in conventional lithography. Further, etch selectivity between block copolymers and their etch resistance for subsequent pattern transfer are generally low.

Materials used to fabricate masters are usually hard, brittle and opaque (except for glass and quartz), and thus are not readily compatible with cell culture and bioimaging techniques. To faithfully transfer surface structures from masters to compliant, transparent materials compatible with cell culture and bioimaging, there is often a need for an intermediate substrate or stamp. Generating stamps usually involves replica molding and related molding techniques using a simple casting procedure of organic polymers on masters followed by thermal or UV curing. Replica molding is a practical method for fabricating structures as small as $500 \mathrm{~nm}$ with accuracy in vertical dimension of $100 \mathrm{~nm}$. The most popular material for replica molding is polydimethylsiloxane (PDMS) (Sylgard 184 PDMS), an elastic, transparent, and biocompatible material. While 184 PDMS works well for replicating structures on a size scale of $500 \mathrm{~nm}$ and larger (Figure 1c), its intrinsic compliance cause shallow relief features of a stamp to deform, buckle, or collapse; in addition, these relief structures tend to deform upon release from the master because of surface tension, makes it lose high fidelity in replicating nanoscale features. To address this issue, harder materials such as UV-curable polyurethane acrylate (PUA) and alternative siloxane polymers such as a polymeric composite based on vinyl and hydrosilane end-linked polymer ("hard" PDMS or h-PDMS) have been applied to replicate highdensity patterns down to a 20-nm scale (Figure 1d). ${ }^{[58-60]}$

Although stamps generated from replica molding can be used directly as synthetic surfaces with micro/nanoscale topographical cues, it is the transfer of micro/nanoscale structures from stamps to secondary synthetic substrates (such as polymers, hydrogels, and glasses) that enables the versatility and popularity of soft lithography (Figure 1a-d). Many techniques have been developed for transferring micro/nanoscale structures from stamps to secondary surfaces, including replica molding, hot embossing, micromolding in capillary, and solvent-assisted micromolding. ${ }^{[38,55]}$ The mechanical compliance of stamps made in elastomeric materials such as PDMS enables conformal contacts of stamps to uneven or even curved surfaces. ${ }^{[61]}$ In addition, using stamps with easily deformable surface structures such as a thin wire or membrane, one can even fabricate curvilinear microstructures with secondary micro/nanoscale topography on their surfaces (Figure 1b). ${ }^{[61]}$

Soft lithography is mainly used for transferring micro/ nanoscale patterns from stamps to secondary surfaces, like PDMS, gold and hydrogel. ${ }^{[38,62]}$ However, it is sometimes desirable to transfer foreign micro/nanostructured materials directly onto a substrate to generate a hybrid material. As an example, the liquid-bridge-mediated nanotransfer molding (LB-nTM) technique recently reported by Hwang and colleagues achieved wafer-scale printing of $3 \mathrm{D}$ patterned nanoscale structures of different materials onto silicon and polymeric substrates (Figure 1e,f). ${ }^{63]}$

Soft lithography and its derivative technologies have been proven as powerful and convenient methods to generate extracellular micro/nanoscale topographical structures in regular patterns. Their applications to study topography-sensitive cell phenotypes and behaviors will continue in the foreseeable future. Integration of soft lithography and its derivative technologies with different functional biomaterials will provide novel foundations for innovative functional biomaterials incorporating unique topographic features for studies of mechanosensitive cell behaviors.

Micro/nanoscale Topography in A Short-range Order. Regularly patterned micro/nanoscale topographies can provide well-defined extracellular substrates and geometries for mechanistic studies of cell-microenvironment interactions under the influence of single or a combination of ECM structural parameters. ${ }^{[64]}$ Studies in recent years have also succeeded in developing low-cost methods for fabrication of micro/nanoscale topography on surfaces that mimics irregular structures, patterns and orders of the ECM surrounding cells in vivo. The fibrous nature of the in vivo ECM, for example, can be recapitulated by coating substrates with micro/nanoscale fibers using electrospinning (Figure 2a,b). ${ }^{[65-71]}$ To initiate electrospinning, a high voltage is applied to a polymer precursor solution that charges the fluidic body, within which a counteraction between

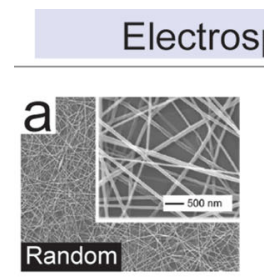

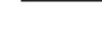

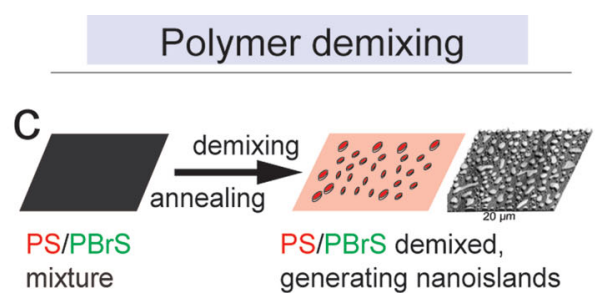

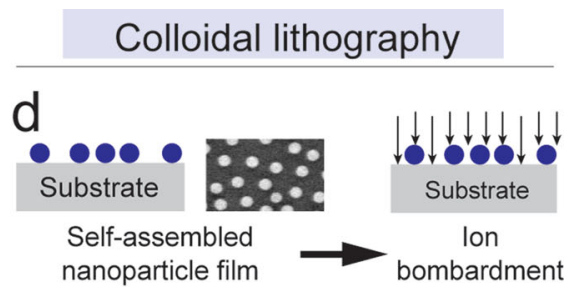

Figure 2. Fabricating micro/nanotopography in a short-range order. (a,b) SEM images of electrospun substrates with poly( $(\varepsilon$-caprolactone) (PCL) nanofibers in a random (a) and highly aligned (b) fashion, respectively. ${ }^{[65]}$ Reproduced with permission. ${ }^{[65]}$ Copyright 2009, Elsevier. (c) Schematic of polymer demixing and SEM image of nano-scale islands fabricated with this method. ${ }^{[76]}$ PS-covered nano-islands were formed during the demixing process of a homogeneous PS/PBrS mixture undergoing annealing. Adapted with permission. ${ }^{[76]}$ Copyright 2004, Elsevier. (d) Schematic of colloidal lithography and SEM image of a self-assembled nanoparticle film used as mask for lithography. ${ }^{[78]}$ The nanoparticles protected materials underneath from the ion bombardment during lithography, and thus generated nano-islands. Adapted with permission. ${ }^{[78]}$ Copyright 2003, Elsevier. 
electrostatic repulsion and surface tension breaks the fluid surface, forms the Taylor cone and extrudes a continuous fine filament toward a grounded collecting substrate. During extrusion, the fiber is further extended and thinned as a result of repulsion from its surface charges, till it reaches and deposits onto the collecting substrate. The diameter of electrospun fibers can vary from above $5 \mu \mathrm{m}$ down to $10 \mathrm{~nm}$, depending on molecular weight and concentration of the polymer, electric potential, flow rate, solution properties and other experimental parameters. Nanoscale fibers with a diameter $<200 \mathrm{~nm}$ usually require some nontrivial technical refinement of the electrospinning instrument and tuning of experimental parameters. Importantly, the intrinsic structural order of electrospun fibrous network can be tuned from completely random (Figure 2a) to a highly aligned fashion (Figure 2b), making it useful to mimic aligned as well as randomly distributed ECM protein fibrils in vivo to induce functional cellular phenotypes (e.g., spontaneous beating of cardiomyocytes) and stem cell differentiation in vitro. ${ }^{65,70,71]}$ In addition to electrospinning, microscale fibrous substrates with various levels of filament density and alignment have been successfully developed by utilizing shear flow deposition within a microfluidic channel, making it compatible with other microfluidic cell culture components and systems. ${ }^{\text {72,73] }}$

The architectural nature of micro/nanoengineered fibrous matrices makes them especially suitable for multiplexed surface functionalization via designing the chemistry of fibers. For instance, electrospun nanofibers dually functionalized with a ECM protein (laminin) and a soluble factor (basic fibroblast growth factor, bFGF) have been fabricated to study the synergistic effect of ECM topography and biochemical cues in guiding neurite outgrowth and fibroblast migration. ${ }^{[74}$ Developing novel strategies to achieve multiplexed, dynamic surface functionalization of micro/nanoscale fibers using electrospinning should broaden its functional capability and future applications in tissue engineering by a precise control of cell-microenvironment interactions and thus directing cellular behaviors.

Besides fibrous surfaces, nanoscale island geometries have also been generated to control micro/nanoscale topography in vitro. Two methods for generating nanoscale islands on surfaces - polymer demixing and colloidal lithography - have been recently developed based on spontaneous pattern formations at a macromolecular scale. In polymer demixing (Figure 2c), nanoscale islands are generated by a spontaneous phase separation process during annealing and demixing of a polystyrene/poly(4-bromostyrene) (PS/PBrS) or poly(L-lactic acid)/ polystyrene (PLLA/PS) mixture. ${ }^{[75-77]}$ In colloidal lithography (Figure 2d), a self-assembled thin film of nanoparticles is used as a lithographical mask, which can shield surface areas underneath nanoparticles from ion bombardment during the following etching step and thus generate nanoscale islands on the surface. ${ }^{[78,79]}$

Both polymer demixing and colloidal lithography rely on spontaneous assembly of either polymers or nanoparticles on surfaces to generate micro/nanoscale topography. The physical nature of such self-assembly processes dictates that there is a short-range order in the micro/nanotopographical patterns generated by these two methods.

Completely Random Micro/nanoscale Topography. To achieve a better control over fabrication of micro/nanoscale topography in a less complex and expensive manner, researchers have taken resort to bottom-up fabrication strategies to generate completely random micro/nanoscale topography from the atomic scale up. So far, researchers have successfully applied reactiveion etching (RIE), ${ }^{[80-82]}$ nanotube growth using anodization ${ }^{[83]}$ and spontaneous galvanic displacement reaction (SGDR) ${ }^{[84]}$ to shape nanoscale topographic landscapes on substrate surfaces in an atom-by-atom manner and with a nanometer resolution. In brief, RIE can effectively generate nanoroughness on silicabased glass surfaces with a nanometer resolution based on a process of ion-enhanced chemical reaction and physical sputtering (Figure 3a). Small concentrations of impurities such as $\mathrm{Al}, \mathrm{K}$, and $\mathrm{Na}$ in the silica glass can result in accumulations of less volatile species (such as AlF3, KF, NaF) on the glass surface during RIE, which can be backscattered onto the glass surface and form randomly distributed small clusters to shield the glass surface from bombardment and reaction with reactive ions. These compound clusters effectively generate the so-called "micromasking" effect that can randomly shadow the glass surface and thus result in nanoscale roughening of the glass surface during RIE (Figure 3b). ${ }^{[82]}$ Using electrochemical anodization in fluorine containing electrolytes, ordered nanotube arrays can be synthesized in titanium oxide $\left(\mathrm{TiO}_{2}\right)$, a biocompatible material often used in orthopedic and dental implants (Figure 3c-e). Advance in synthetic techniques makes it possible to prepare $\mathrm{TiO}_{2}$ nanotube arrays with various pore diameters $(10-110 \mathrm{~nm})$, thicknesses $(200-1000 \mu \mathrm{m})$, and wall
Reactive lon Etching

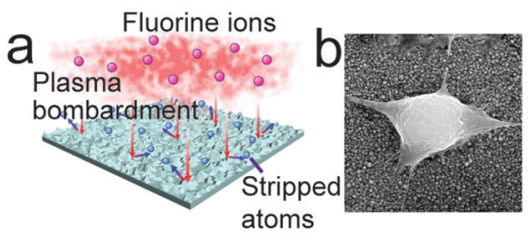

Fabrication of nanomaterials

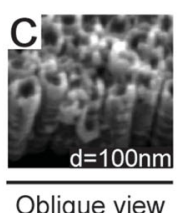

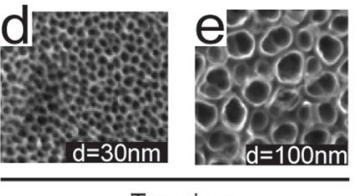

Top view
SGDR

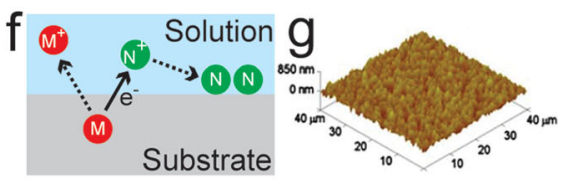

Figure 3. Fabricating random nanotopography. (a) Schematic of generating nanoroughness onto a substrate using reactive ion etching (RIE), ${ }^{[82]}$ during which atoms were randomly and unevenly stripped off the substrate by plasma bombardment. (b) SEM image of a cancer cell on the nanorough substrate. Reproduced with permission. ${ }^{[82]}$ Copyright 2012, American Chemical Society. (c-e) SEM images of arrays of titanium dioxide (TiO 2 ) nanotubes of different diameters, which were used to dictate differential fate of hMSCs. ${ }^{[83]}$ Reproduced with permission. ${ }^{[83]}$ Copyright 2009, United States National Academy of Sciences. ( $f$ ) Schematic of the spontaneous galvanic displacement reaction (SGDR), during which solute metallic ions accept electrons from substrate metallic atoms and then deposit onto the substrate as nano-scale clusters. (g) AFM image of a nanotopographical substrate created by SGDR and used for studying the response of neurons. ${ }^{[84]}$ Reproduced with permission. ${ }^{[84]}$ Copyright 2010, United States National Academy of Sciences. 
thicknesses (7-34 nm). ${ }^{[83]}$ SGDR is routinely used for electroless plating of metals in bulk materials and is also applied to make microcontacts in electronics industry. In SGDR, a metallic substrate atom donates an electron to a solute metallic atom, which then deposits onto the substrate and forms randomly distributed nanoscale islands on the substrate (Figure 3f,g). ${ }^{[84]}$

Topography-Sensitive Cell Phenotypes. A large set of micro/ nanoscale topographical substrates have so far been developed, and they have helped reveal several important aspects in cellular sensing of extracellular topographical cues: (1) Contact guidance, a phenomenon where cell morphology and migration is preferably aligned with polarized nanoscale fiber and ridge structures. Since its first description in early 1990s, contact guidance has been one of the first as well as one of the most studied topography-responsive cellular behaviors. ${ }^{[85-87]}$ In addition to structural polarity in nanotopography, anisotropy and gradient of nanotopography have also been found to play a functional role in regulating cell morphology, migration and functions; ${ }^{[58,59,73]}$ (2) Sensing nanotopography via cell adhesions. While the concept of contact guidance was established for polarized nanotopography, recent studies have suggested that adherent mammalian cells are also responsive to non-polarized random, uniform nanotopographical surfaces. On nanorough glass substrates fabricated by RIE, for example, Chen and colleagues observed adherent mammalian cells exhibiting faster initial cell spreading but smaller saturation cell spreading area than the cells seeded on smooth surfaces. ${ }^{[80,82]}$ This observation was consistent with those reported by Dalby and colleagues, ${ }^{[76]}$ where nanoscale islands of different sizes generated by polymer demixing resulted in differential regulations of both short- and long-term cell spreading. In addition, integrin-mediated FAs for cells seeded on nanorough substrates were distributed fairly evenly across the whole cell spreading area, with smaller individual FA size but a greater total FA number, while FAs for cells on smooth surfaces were almost exclusively distributed along cell periphery with larger individual FA size and a less total number of FAs. ${ }^{[0,82,84]}$ These observations suggest that the intrinsic nanoscale topography, in addition to structural polarity of surface topography, can play a functional role in regulating cellular behaviors, likely through their direct effect on cell adhesion assembly and signaling; (3) Topography-sensitive cell functions. Cell adhesions and adhesionmediated intracellular signaling cascades are known important to regulate many long-term cellular behaviors, such as survival, proliferation and differentiation. ${ }^{[19,24,88]}$ Thus, it is not surprising that nanotopography, which can affect cell adhesion assembly and signaling, can influence many important cell behaviors. Many recent studies, for example, have confirmed the regulatory role of nanotopography for lineage commitment and differentiation of stem cells, including mesenchymal stem cells (MSC) ${ }^{[68,83,89,90]}$, neural progenitor cells (NPCs), ${ }^{[91]}$ neural stem cells (NSCs), ${ }^{[66]}$ human induce pluripotent stem cells (iPSCs) ${ }^{[92]}$ and mouse ${ }^{[65]}$ and human ${ }^{[80,93]}$ embryonic stem cells (ESCs), using micro/nanoscale topographical substrates fabricated by EBL, ${ }^{[89,90]}$ laser interference lithography, ${ }^{[92]}$ soft lithography, ${ }^{[91]}$ electrospinning, ${ }^{[65,66,68]}$ electrochemical anodization ${ }^{[83]}$ and RIE. ${ }^{[80]}$ Another notable example was demonstrated by Kim and colleagues, where functions of cardiac tissue constructs in terms of action potential and contraction were shown to be sensitive to nanoscale topography. ${ }^{[95,96]}$
Even though many micro/nanoengineered topographies have been developed and many topography-sensitive cellular phenotypes have been documented, the molecular mechanism of cellular sensitivity to micro/nanoscale topography remains incompletely understood. Given that FAs are multifunctional organelles mechanically connecting intracellular actin cytoskeleton to the ECM and FAs are mechano-sensitive and -responsive and are known as a scaffold for intracellular signaling, it is plausible that adherent cells sense and respond to nanotopographical cues through actively modifying FA assembly and signaling. Involvement of FA signaling in cellular sensing of topography was supported by a recent study demonstrating that nanoscale grating-induced neural differentiation of human MSCs were mediated by focal adhesion kinase (FAK), a FA signaling protein, as inhibition of FAK abrogated topographysensitive neural differentiation of human MSCs. ${ }^{[97]}$ It was further echoed by another recent study showing that nanotopographical manipulation of FAs and FAK phosphorylation was correlated with the enhancement of human NSCs differentitation. ${ }^{[9]}$ Recent efforts from Dalby and colleagues using high-dimensional biology tools (genomics and metabolomics) and systems biology approaches have further provided insights on critical biochemical pathways such as ERK 1/2 and JNK involved in topography-sensitive long-term maintenance of human MSC phenotype and multipotency. ${ }^{[89,90,94]}$ Another potential future direction will be to leverage recent developments of super-resolution microscopy methods ${ }^{[99]}$ with a singlemolecule resolution to examine in situ how nanoscale architecture and signaling of integrin-mediated cell adhesions are affected by micro/nanoscale topological cues.

\section{1.2. Engineering Mechanical Stiffness of Extracellular Matrix}

Mechanical stiffness of the ECM is an intrinsic matrix mechanical property that characterizes the ability of the ECM to resist deformation in respond to a sustained mechanical force acting onto it. In vivo, mammalian cells reside in microenvironments with a broad range of mechanical stiffness spanning from $\sim 0.1 \mathrm{KPa}$ (brain-like soft tissue) to $\sim 50 \mathrm{KPa}$ (bone-like hard tissue). ${ }^{[13,21]}$ Under pathological conditions, mechanical properties of diseased tissues can be altered significantly. For example, cancer cells are surrounded in tumor stroma that is 5-20 times stiffer than normal tissue. ${ }^{[100]}$ It is well recognized that cancer development, like organogenesis during embryonic development and tissue repair in adult mammals, is regulated by interactions between cancer cells, activated stromal cells, and biochemical and biophysical components (such as stiffness) of the ECM..$^{[3,5,17]}$

The ability of adherent mammalian cells to sense and respond to ECM mechanics has been demonstrated very recently, yet its implications for functional tissue engineering and regenerative medicine have already generated tremendous excitement. Many mammalian cell types, including both adult and pluripotent stem cells such as skeletal muscle stem cells, hematopoietic stem cells, adult neuron stem cells and embryonic stem cells, have been studied for their mechanoresponsive behaviors to ECM mechanics in both 2D and 3D microenvironments. ${ }^{[101]}$

For adherent cells seeded in both 2D and 3D microenvironments, the cells will mechanically contract and pull against the 
surrounding matrix (cellular contractile force) resulting in a force balance between an intracellular myosin-based contraction force and a resisting force originated from the deformation of the ECM. ${ }^{[19,102]}$ Such tensional homeostasis in the intracellular cytoskeleton has a key role in regulation of basic cellular functions, such as cell proliferation, apoptosis, adhesion, and migration. Deregulation of tensional homeostasis in cells contributes to pathogenesis of several human diseases, such as atherosclerosis, osteoarthritis and osteoporosis, and cancer. The degree the ECM deforms in respond to cellular contractile force is dictated by mechanical stiffness or bulk modulus of the ECM. Tensional homeostasis is also believed to be critically involved in cellular rigidity-sensing mechanisms, by actively regulating the mechanical force balance transmitted across the ECM-integrin-cytoskeleton to regulate integrin-mediated adhesion signaling (such as FAK and Src signaling) to coordinate downstream integrated cell function. ${ }^{[14,21,22,100,103,104]}$

Recent developments of micro/nanoengineering and materials science have provided many synthetic functional biomaterials as platforms for researchers to modulate mechanical properties of in vitro cell microenvironment from the molecular scale to the tissue scale in both 2D and 3D contexts. These synthetic functional platforms have helped researchers to elucidate many important aspects of cellular mechano-sensing and -transduction mechanisms (especially how cells sense and respond to changes of ECM stiffness) as well as inspire novel strategies to translate new knowledge of mechanobiology to advance biomaterial design for regenerative medicine.

Elastomeric Micropost Arrays on 2D Surfaces. An ideal way of engineering ECM stiffness requires a material system that can achieve isolation of ECM mechanics from other structural and molecular-scale material properties, such as surface porosity and topography, surface chemistry, backbone flexibility and binding properties of immobilized adhesive ligands. Following this idea, a set of synthetic functional biomaterials composed of arrays of elastomeric microposts have been developed using microfabrication and replica molding with PDMS in the last decade (Figure 4). ${ }^{[105-111]}$ An elegant feature of the PDMS micropost array lies in the simple geometrical modulation of each post's bending flexibility in response to a lateral force that closely reflects the nature of cellular contractile force for cells seeded on a 2D surface. Dictated by the beam theory, bending rigidity or the spring constant, $K$, of each cylindrical micropost scales with its diameter $d$ and height $L$ as $K=3 \pi E d^{4} /\left(64 L^{3}\right)$, where $E$ is the bulk Young's modulus of PDMS (Figure 4a). Spring constant $K$ of the PDMS micropost array can be further converted into an equivalent Young's modulus $E_{\text {eq }}$ of a continuous elastic substrate as $E_{\mathrm{eq}}=9 K /(2 \pi d)$. Thus, by geometrically modulating post structural parameters including post height and diameter, it is straightforward to control structural stiffness of the PDMS micropost, as cellular contractile forces are generally applied in the 2D plane of substrate surface where the cells are seeded and thus mostly probe bending flexibility of the PDMS micropost (Figure 4b,c).

The elastomeric PDMS micropost array can be batch fabricated in a large volume using microfabrication and replica molding. ${ }^{[108,110]}$ Detailed description of a standard method for fabrication of PDMS micropost arrays can be found elsewhere. ${ }^{[108]}$ Briefly, fabrication of PDMS micropost arrays involves first patterning photoresist on a Si wafer using contact

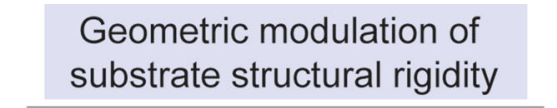

Geometric modulation of
substrate rigidity anisotropy
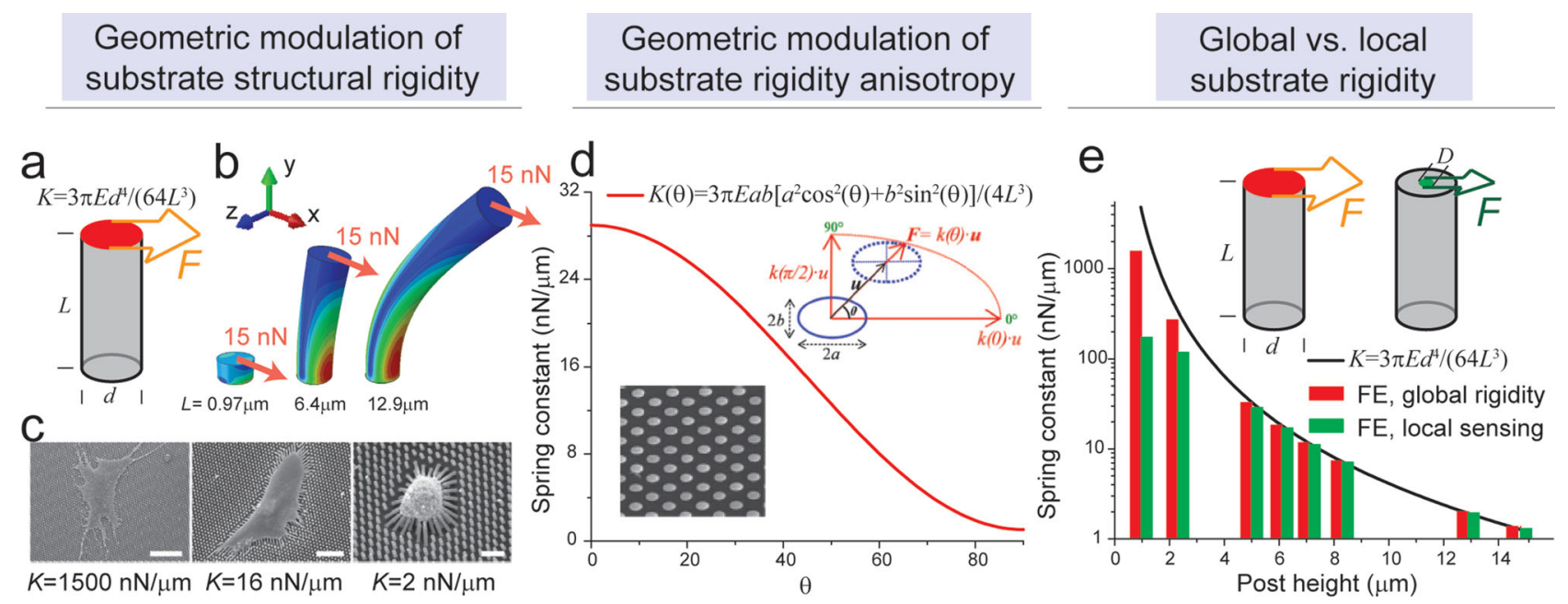

Figure 4. Modulation of substrate mechanics by micro-post arrays. (a) Schematic of the beam theory, where the flexure displacement is proportional to the lateral force via a factor called spring constant, $K$. The spring constant is determined by the material and geometry of the beam and thus enables geometrical modulation of the effective stiffness of the substrate. (b) Finite element (FE) simulation results of beams of different heights subject to the same amount of lateral forces $(15 \mathrm{nN})$ applied over the tips, illustrating that the higher the beam the easier it bends. (c) SEM images of hMSCs cultured on micropost arrays with different spring constants, demonstrating cell shape was sensitive to the structural rigidity of the substrate. ${ }^{[107]}$ Reproduced with permission. ${ }^{[107]}$ Copyright 2010, Nature Publishing Group. (d) Theoretical prediction of the anisotropic spring constant of a beam with oval cross-section, where $\theta$ is the angle between the force and the longitudinal axis of the cross-section. ${ }^{[106]}$ The aspect ratio of the ellipse, $a / b=3$, was used for the plot. Inset: SEM image of the top surfaces of microposts with oval cross-sections. Adapted with permission. ${ }^{[106]}$ Copyright 2007, United States National Academy of Sciences. (e) FE simulation results of the effect of local force on the overall effective spring constant of a beam under lateral force applied to its top. When the same amount of lateral force was applied just within a nano-scale local area of the post top $(D / d=1 / 10, d=1.8 \mu \mathrm{m}$, $E=2.5 \mathrm{MPa}$ ), rather than over the entire post tip, the effect of local rigidity sensing by local force generally decreased the effective spring constant of the beam. Such effect of local sensing is expected to be greater when the material stiffness of the beam becomes smaller. 
or projection photolithography before the wafer is dry etched using deep reactive ion-etching (DRIE) to generate an array of microposts or microscale holes in the Si wafer. Heights or depths of microposts and holes in the Si wafer are easily controlled by varying etching time during DRIE. Geometries of the microposts or holes in the Si wafer are transferred into PDMS post structures using single replica molding (using the Si microscale hole array) or double replica molding (using the Si micropost array). Importantly, as an independent step, the PDMS micropost array is functionalized and rendered adhesive by microcontact printing of adhesive molecules across micropost tops. Subsequent treatment with Pluronic, a nonionic surfactant, or bovine serum albumin (BSA) to block nonspecific protein adsorption to post shafts can ensure that cells seeded on the PDMS micropost array are only able to adhere to the micropost tops and not crawl in between. Fabrication of and surface functionalization strategy for the PDMS micropost array allow for the structural stiffness or spring constant of the micropost to be modulated simply by varying post height while keeping all other aspects of the substrate such as surface chemistry and ligand density unchanged. ${ }^{[108]}$ The PDMS micropost array reported so far can have a post diameter from submicron to about $10 \mu \mathrm{m}$ and a post height to diameter ratio up to about 20, spanning a more than 10000 -fold range of post spring constant from $0.06-4000 \mathrm{nN} \mu \mathrm{m}^{-1}$ (the equivalent bulk modulus $E_{\text {eq }}$ ranging from $10 \mathrm{~Pa}$ to $3 \mathrm{MPa}$ ), much broader than is currently achievable with natural or synthetic hydrogels.

It is worth noting that in addition to Sylgard 184 PDMS, other PDMS derivatives have also been developed recently to fabricate micropost structures. One recent study by Sun and colleagues added benzophenone as a photoinhibitor in PDMS to generate photosensitive PDMS (photoPDMS). ${ }^{[112]}$ Conventional PDMS consists of repeating -OSi(CH3)2- units. The PDMS base monomer is vinyl terminated, while the crosslinkers are methyl-terminated and contain silicon hydride -OSiHCH3- units. During curing, PDMS base monomers crosslink via a reaction between the monomer vinyl groups and the silicon hydride groups of the crosslinkers to form $\mathrm{Si}-$ $\mathrm{CH} 2-\mathrm{CH} 2-\mathrm{Si}$ linkages. For photoPDMS, benzophenone is added into PDMS to act as a photoinhibitor, which generates free radicals under UV light exposure. Benzophenone radicals can abstract a hydrogen atom from a suitable hydrogen donor and thus react with both silicon hydride units in PDMS crosslinkers and vinyl groups in PDMS monomers. This reaction prevents crosslinking reactions between PDMS monomers and crosslinkers and thus decreases the degree of PDMS crosslinking during post-exposure bake. Sun and colleagues demonstrated that the bulk Young's modulus of photoPDMS could be conveniently controlled within a broad range (from 0.027 to $2.48 \mathrm{MPa}$ ) by modulating the PDMS crosslinker to base monomer ratio, UV light exposure time, and post-exposure baking time. Biocompatibility and cytotoxic effect of photoPDMS was also evaluated with a negligible effect of benzophenone in photoPDMS detected on cell viability and growth. Using photoPDMS, Sun and colleagues fabricated photoPDMS micropost arrays for multiscale study of mechanoresponsive cellular behaviors, and their results supported that adherent mammalian cells could sense and respond to changes of substrate rigidity at a sub-focal adhesion resolution.
Instead of using PDMS and its derivatives, recently, Rahmouni and colleagues developed a novel elastomeric micropost array made of poly(ethylene glycol) (PEG) diacrylate hydrogel using microscope projection photolithography via UV-mediated photo-crosslinking of PEG prepolymer. ${ }^{[113]}$ PEG microposts with a post diameter down to $2 \mu \mathrm{m}$, a post centerto-center distance of $5 \mu \mathrm{m}$, and a post height to diamter ratio up to 10 were successfully fabricated. Importantly, a significant advantage of PEG microposts was the broad intrinsic material stiffness of PEG hydrogel $(1 \mathrm{KPa}-1 \mathrm{MPa})$, which is difficult to achieve using PDMS or photoPDMS. Furthermore, via surface functionalization using block copolymer lithography with gold nanoparticles, the authors grafted onto the PEG micropost nanoscale patterns of adhesive ligands that could selectively bind $\alpha_{V} \beta_{3}$ and $\alpha_{5} \beta_{1}$ integrins, thus providing a novel biomaterial platform for studying the functional roles of integrin receptors, nanoscale arrangements of adhesive ligands, and traction forces in cellular sensing of substrate rigidity.

In addition to changing cylindrical micropost height or diameter to modulate structural stiffness of PDMS microposts, ${ }^{[109]}$ one can also integrate PDMS microposts of different heights and diameters on the same substrate with post tops on the same 2D surface to spatially pattern substrate stiffness or stiffness gradients. ${ }^{[109,114]}$ Another novel example was to generate oval-shaped PDMS microposts to introduce structural anisotropy to the micropost geometry to achieve anisotropic structural stiffness. ${ }^{[106,114,115]}$ For example, theoretical results shown in Figure 4d illustrates that an oval-shaped micropost with the major and minor axis length ratio of 3 could render a tenfold change in the spring constant of the micropost along different angular directions. ${ }^{[106]}$

Although the structural stiffness of elastomeric microposts is governed mostly by the bending rigidity or spring constant of the post, it is by no means the only mechanical property a cell could sense when seeded on the posts. The spring constant of an elastomeric micropost is evaluated assuming that there is a uniform stress applied across the post top surface. However, the smallest cell adhesion structure that can transmit intracellular contractile forces from a cell to the underlying surface is nascent FAs of a diameter about 200-500 nm and much smaller than the nominal post diameter ${ }^{[116]}$ In addition, single integrin heterodimers, which cluster to form force-transmitting FAs during cell spreading and migration, are of sizes down to $10-50 \mathrm{~nm} .{ }^{117]}$ Thus, it is likely that adherent cells, during cell spreading and migration, will exert forces to the micropost tops through adhesion areas much smaller than the micropost top surface, and thus the cells will be able to sense the local intrinsic nanoscale material stiffness (i.e., bulk Young's modulus) of the PDMS micropost (Figure 4e). ${ }^{[112,118]}$ Our finite element simulation (FEM) results in Figure $4 \mathrm{e}$ indeed demonstrated that the adhesion area through which a contractile force was applied had a non-negligible effect on the overall effective spring constant of the PDMS micropost, even when the bulk Young's modulus of PDMS remained as $2.5 \mathrm{MPa}$. It is expected that when softer elastomeric materials are used to fabricate micropost structures, ${ }^{[112]}$ the effects of local force application and FA size can become much more significant and maybe even dominate the effect of the overall structural stiffness of the micropost. 
Static cross-linking

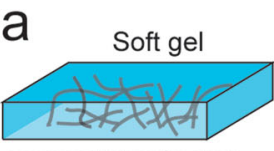

Low crosslinker fraction

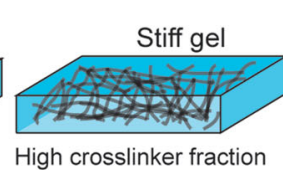

High crosslinker fraction
Photo-assisted stiffening

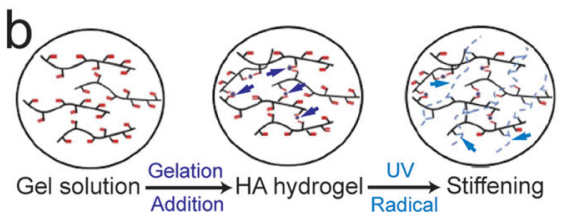

Photo-assisted softening

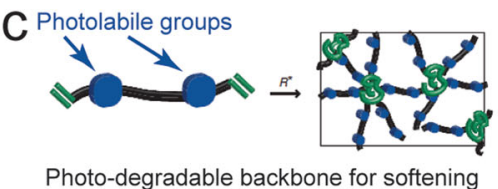

Figure 5. Modulation of substrate mechanics by hydrogel cross-linking. (a) Schematic of the dependence of hydrogel stiffness on the fraction of crosslinkers. (b) Schematic of gelation and photo-assisted stiffening of a methacrylated hyaluronic acid (MeHA) gel, wherein the gelation was achieved by addition reaction and the stiffening was introduced via UV-initiated radical polymerization. ${ }^{[130]}$ Reproduced with permission. ${ }^{[130]}$ Copyright 2012 , Nature Publishing Group. (c) Schematic of photo-assisted softening of a PEG-based gel made of crosslinking units with photo-degradable backbones. ${ }^{[133]}$ Reproduced with permission. ${ }^{[133]}$ Copyright 2010, Nature Publishing Group.

Crosslinking Hydrogels in Both 2D and 3D Microenvironments. While elastomeric micropost arrays were fabricated mostly with PDMS owing to its stable chemical composition and ease of use, synthetic hydrogels, such as polyacrylamide (PAA)- and PEGbased hydrogels, have also been widely used for studying mechano-sensitive and -responsive properties of adherent cells. ${ }^{[13,119-122]}$ By simply adjusting the ratio of acrylamide to bis-acrylamide (Figure 5a), for example, one could change the crosslinker density in PAA gels and thus modulate its bulk Young's stiffness spanning 3 orders of magnitude $(0.1 \mathrm{KPa}-200 \mathrm{KPa}) .{ }^{[13,119,123]}$ In contrast to PAA gels, which remain elastic when modulating its bulk stiffness, PDMS increases its viscoelasticity when decreasing its stiffness. ${ }^{[124]}$ Thus, it requires attention when comparing results obtained from mechanosensitive cells seeded on "soft" and "stiff" PDMS, ${ }^{[124]}$ as one needs to carefully interpret which mechanical property - either elasticity under fast loading or viscosity under slow loading - truly elicits mechanosensitive behaviors of adherent cells. In addition to PAA- and PEG-based hydrogels, recently, a versatile control of the static mechanical properties of gelatin metharylate (GelMA) hydrogel was demonstrated via incorporating carbon nanomaterials within the GelMA hydrogel. ${ }^{[125,126]}$ Even though its porosity and surface hydrophobicity may vary with its bulk stiffness, presenting a potential issue for interpreting experimental results, PAA- and PEG-based hydrogels and their derivatives are still the most popular and one of the most effective biomaterials for studying cellular responsiveness to matrix stiffness, in both 2D and $3 \mathrm{D}$ contexts. To introduce rigidity gradients into synthetic hydrogels, a simple method had been developed by Lo and colleagues by placing adjacent to each other two PAA droplets of different acrylamide/bis-acrylamide ratio. ${ }^{[127]}$ More advanced methods, such as those using microfluidics-assisted or UV-based rigidity patterning for hydrogels, required more complex experimental setups; however, these methods allowed a better control and generation of rigidity patterns and gradients in synthetic hydrogels. ${ }^{[123,128,129]}$

A unique feature associated with synthetic hydrogels is the possibility for dynamic postgelation control of crosslinking in hydrogels in real time even in the presence of cells. This feature opens the door for novel synthetic hydrogels to dynamically regulate hydrogel properties in situ, making them a better mimic for in vivo 3D ECM remodeling (e.g., during embryo development, fibrosis and cancer metastasis) as well as allowing studies of cellular responses to dynamic changes in matrix properties. Recent investigations by two separate research groups have made exciting progress along this direction by using photo-assisted stiffening and softening strategies to dynamically modulate mechanical stiffness of synthetic hydrogels in the presence of cells.

In the photo-assisted stiffening strategy developed by the Burdick group, ${ }^{[47,130,131]}$ sequential crosslinking was achieved as a route for in situ hydrogel stiffening for hyaluronic acid (HA) macromers functionalized with methacrylates (methacrylated $\mathrm{HA}$, or MeHA). HA is a linear polysaccharide composed of alternating $d$-glucuronic acid and $N$-acetyl-d-glucosamine. Since $\mathrm{HA}$ is a natural component of the ECM and is involved in many biological processes, HA has been chemically modified in a variety of ways to form hydrogels with tunable properties (for example, hydrophobicity, degradation) towards the development of biomaterials for tissue engineering and regenerative medicine. ${ }^{[47]}$ As methacrylates can react with both thiols and radicals for crosslinking, MeHA allows sequential crosslinking (addition then light-mediated crosslinking) to create hydrogels that stiffen (for example, 3-30 kPa) in the presence of cells (Figure 5b). During the initial gelation of MeHA hydrogel, dithiolreitol (DTT) was added to MeHA solution as crosslinkers, and methacrylates reacted with thiols on the DTT. The stiffness of MeHA hydrogel was regulated during this initial gelation by controlling the amount of DTT. Subsequent stiffening of the gel was achieved by radical polymerization, when the hydrogel was swollen with a photoinitiator and exposed to UV light. ${ }^{[130]}$ In a more recent study from the same group, proteolytically (matrix metalloproteinase, MMP) cleavable crosslinkers were added to MeHA hydrogels during the initial gelation process, allowing gel degradation in the presence of cells during long-term cell culture. ${ }^{[131]}$ In this work, UV-assisted radical polymerization was also performed optionally after initial gelation process, to inhibit proteolytic degradation during long-term cell culture. ${ }^{[131]}$

The photo-assisted softening strategy developed by the Anseth group used photodegradable PEG-based hydrogels through rapid UV-polymerization of cytocompatible macromers for remote manipulation of gel properties in situ. ${ }^{[132-135]}$ Synthetic photodegradable monomers containing photolabile groups were created as fundamental structural elements to generate photolytically degradable hydrogels whose physical or chemical properties were tunable temporally and spatially with light on demand. In their method, a critical step was to couple a nitrobenzyl ether-derived moiety with a high photolytic efficiency to PEG-bis-amine to create photocleavable crosslinking diacrylate macromer from which PEG-based 
photodegradable hydrogels were synthesized (Figure $5 \mathrm{c}$ ). Since the photolabile moiety was incorporated directly within the network backbone, the hydrogel itself was cleaved upon light exposure, decreasing the local network crosslink density and resulting in macroscopic property changes such as mechanical stiffness in the presence of cells. In addition, coupling photodegradable monomers with fibronectin-derived adhesive peptides made it possible to functionalize the photodegradable PEG hydrogel with photoreleasable cell adhesion motifs, allowing the local biochemical microenvironment tunable with light for encapsulated cells. ${ }^{[133,134]}$ Photo-assisted softening and degradation of the photodegradable PEG hydrogels could be completed in almost real time (within minutes or even seconds), and the location could be precisely controlled using focused laser irradiation. In contrast to photo-assisted hydrogel stiffening, photoassisted hydrogel softening and degradation not only enabled studies of cellular responses to dynamic matrix softening, but also achieved in situ local microenvironment patterning and re-structuring within a cell-laden 3D hydrogel (we will discuss more about this perspective in section 2.1.3). ${ }^{[134,135]}$

Molecular Tethering on 2D Substrates. Unlike PDMS, which is effectively a continuous medium with its average pore size of less than $1 \mathrm{~nm}$, synthetic hydrogels like PAA- and PEGbased hydrogels have a nanoporous structure with its average pore size inversely proportional to its poroelastic modulus (e.g., $15 \mathrm{~nm}$ and $5.8 \mathrm{~nm}$ pore sizes for $0.5 \mathrm{KPa}$ and $115 \mathrm{KPa}$ PAA gels, respectively). ${ }^{[124]}$ Given the nanoporous nature of synthetic hydrogels, adhesive ligands immobilized onto the gels are anchored on a nanoscale porous grid with deformable backbones (Figure 6a). ${ }^{[124]}$ Thus, for synthetic hydrogels, in addition to the apparent material stiffness (e.g., the poroelastic modulus), molecular-scale material properties, such as surface porosity, polymer backbone flexibility, and binding properties of immobilized adhesive ligands, may need to be taken into account when explaining mechano-sensitive and -responsive cellular behaviors (Figure 6a).

Recently, Trappmann and colleagues ${ }^{[124]}$ developed a molecular tethering material system to investigate specifically effects of surface porosity and polymer backbone flexibility on mechano-sensitive and -responsive cellular behaviors. By adapting a method reported by Aydin and colleagues, ${ }^{[136]}$ Trappmann and colleagues created synthetic PAA-based hydrogels with arrays of gold nanoparticles embedded at the gel surface to present discrete anchoring points at the gold nanoparticles for tethering adhesive ligands. Different anchorage patterns for tethering adhesive ligands were achieved by adjusting gold nanoparticle distances on PAA gels (Figure 6b). Using this synthetic PAA platform, Trappmann and colleagues observed that cellular behaviors on denser gold nanoparticle arrays appeared similar to those on stiffer substrates. In light of molecular tethering mechanics (Figure 6c), Trappmann and colleagues proposed that ECM protein anchorage pattern and hydrogel backbone flexibility, combined with molecular tethering under cellular contractile forces, could create an effective molecular scale stiffness that could only be sensed by contractile cells. ${ }^{[124]}$ The results reported by Trappmann and colleagues not only confirmed the important roles of ECM protein anchorage and tethering in regulating mechanoresponsive cellular behaviors, but also suggested that their effects might occur through cellular sensing of an effective environmental rigidity combining molecular tethering and hydrogel backbone deformation. The nonlinearity of such effective matrix stiffness sensing might present challenges for quantitative characterizations of its effects. However, this novel nanoengineered hybrid biomaterial system with controlled molecular tethering properties could provide a unique strategy to manipulate substrate stiffness at a molecular scale.

Cellular Sensing of Microenvironmental Rigidity. The great ability to modulate ECM stiffness in vitro using micro/nanoengineered functional biomaterials has advanced our knowledge and applications of cellular rigidity-sensing on several important aspects. (1) Rigidity-sensitive cell phenotypes. The arsenal of 2D and 3D matrix with different mechanical stiffness has allowed researchers to demonstrate a large group of rigidity-sensitive cell phenotypes such as cell spreading and FA formation, ${ }^{[21,107,119,137]}$ FA dynamics, ${ }^{[109]}$ architecture of actin

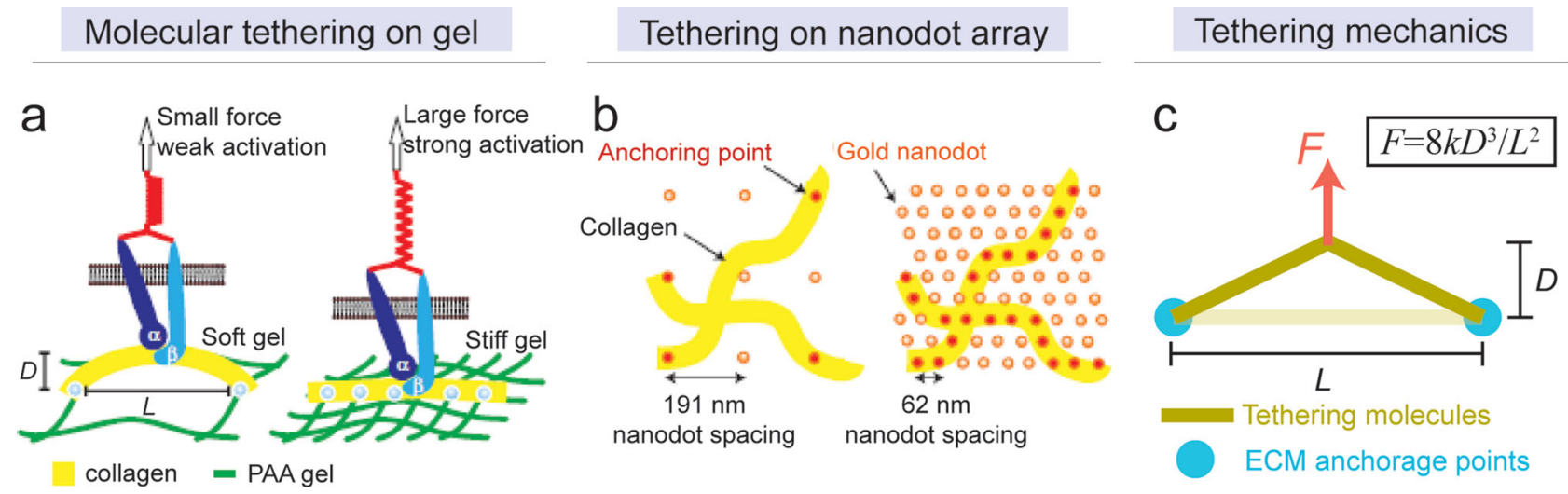

Figure 6. Modulation of substrate mechanics by molecular tethering. (a) Schematic of the anchorage and tethering of collagen on polyacrylamide (PAA) gel of different stiffness and different pore sizes. ${ }^{[124]}$ Collagen molecules having higher anchorage density on stiffer substrate sustained larger tethering force by integrin and its activation. (b) Schematic of different anchorage density of collagen molecules on arrays of gold nanodots with different spacing. ${ }^{[124]}$ Reproduced with permission. ${ }^{[124]}$ Copyright 2012, Nature Publishing Group. (c) A model of molecular tethering mechanics, where the tethering force has a nonlinear relation with the tethering displacement, $D$, and the original length of molecule, $L$, assuming each half of the molecule behaves like a linear spring of constant $k$. 
cytoskeleton $(\mathrm{CSK}),{ }^{[21,138]}$ persistent and directional cell migration (durotaxis), ${ }^{[121,127]}$ stem cell differentiation ${ }^{[13,107,139]}$ and cell proliferation. ${ }^{[140,141]}$ Mechanoresponsive cell proliferation is particularly important for cancer research, as it suggests that by mimicking biomechanical stromal remodeling or tissue/organspecific microenvironmental stiffness, it is possible to reconstitute and implicate a mechanistic role for matrix stiffness in cancer progression and metastasis. ${ }^{[17,103]}$ While cellular rigiditysensing share many common features in 2D and 3D contexts, however, it is important to note that cellular sensing of $3 \mathrm{D}$ matrix "rigidity" actually involves several interdependent microenvironmental factors across molecular and cellular scales: bulk deformability of crosslinked hydrogel as well as molecular backbone flexibility, spatial confinement of the cell in 3D porous environment, spatial presentation of adhesive ligands and degradation of local matrix surrounding the cell. ${ }^{[131,142,143]}$ Therefore, it requires caution to directly compare observations of cellular rigidity-sensing in 2D and 3D contexts. ${ }^{[107,131,142,144,145]}(2)$ Cell mechanotransduction. There are many mechanotransduction mechanisms that the cell can utilize to sense and respond to biophysical cues in the local cellular microenvironment, and likely these mechanisms can work in parallel or synergistically to elicit an integrated cellular response. Among these mechanotransduction mechanisms, two signaling pathways have been mostly studied. The RhoA/ROCK signaling pathway, including its regulation of the "FA-actin CSK-myosin-mediated contractile force" triad ${ }^{[146]}$ and associated signaling crosstalk (e.g., FAK-Src pathway and EGFR/MAPK/ERK pathway), has been shown to be required for many different rigidity-sensitive cell phenotypes and behaviors during development, tissue remodeling and cancer progression. ${ }^{[19,24,50,103,147-150]}$ The Hippo pathway, which is well studied in developmental biology and cancer research, ${ }^{[151]}$ has recently been identified to play an important role in regulating mechano-responsive stem cell differentiation, where nuclear-cytoplasmic translocation of YAP/TAZ transcription factors acts as a rigidity-sensitive step relaying cytoplasmic mechanotransductive signals to cell nucleus to regulate gene expressions. ${ }^{[152-155]}$ More recently, a novel pathway involving chaperone-assisted selective autophagy (CASA) has been proposed for cell mechanotransduction in smooth muscle cells, where the CASA complex such as cochaporone BAG3 is found to mediate a positive feedback between intracellular cytoskeleton tension, autophagic sorting of damaged filamin (an actin CSK crosslinker), and activation of YAP/TAZ nuclear translocation. ${ }^{[156]}$ This study suggests a functional link and coordination between the RhoA/ROCK-CSK contractility signaling axis and the nuclear-cytoplasmic translocation of YAP/TAZ for cellular rigidity-sensing. Another recent study suggested that nuclear lamin-A not only scales in quantity with in vivo extracellular matrix stiffness, but also regulates rigidity-dependent MSC differentiation by controling nuclear remodeling via the retinoic acid (RA) pathway and mediating transcription factors such as serum response factor (SRF) and the Hippo pathway nuclear effector YAP1. ${ }^{[157]}$ Detailed mechanistic understanding of cellular rigidity-sensing and mechanotransduction is not yet available. Future work combining high-throughput genomic and proteomic tools, super resolution live cell microscopy, and novel micro/nanoengineered functional biomaterials will likely provide exciting insights of cellular rigidity-sensing and mechanotransduction down to the molecular level. (3) Stem cell-based cell therapy for regenerative medicine. The recent excitement to control substrate mechanics to direct specific lineage differentiation of cultured human stem cells, such as ESCs, ${ }^{[158]}$ MSCs, ${ }^{[13,131,142,159,160]}$ NSCs, ${ }^{[144,145]}$ HSCs (hematopoietic stem cells) $)^{[161,162]}$ and MuSCs (skeletal muscle stem cells) ${ }^{[163]}$ has revived the promise of stem cells for regenerative medicine in a mechanobiology context. (4) In vitro cancer model. In a recent study, Liu and colleagues observed that soft fibrin hydrogel was able to selectively promote proliferation of tumorigenic cells encapsulated in the hydrogel. ${ }^{[164]}$ This study suggested a novel method for in vitro selection and culture of tumorigenic cells and tumorigenic spheroid. It also suggested a route based on novel functional biomaterials to establish in vitro tumor pathogenesis models for studying cancer progression and metastasis.

\subsubsection{Micro/Nanoscale Patterning of Cell Adhesive Cues}

In addition to its topographical and mechanical properties, the ECM in vivo also presents encapsulated cells with spatially distributed biochemical cues imposed by adjacent cells and the surrounding ECM, such as asymmetrical or local presentation of ligands and adhesion motifs and gradients of soluble factors. Biochemical properties of the ECM and the physical fashion in which they are presented to the cell encapsulated in the ECM are important for regulating dynamic cellular behaviors, such as cell migration and asymmetrical cell division, critical for in vivo embryo development, wound healing, and fibrosis. ${ }^{[165,166]}$ However, the spatially distributed nature of the ECM biochemical cues in vivo is not recapitulated in conventional tissue culture dishes and plates with homogeneous uniform surface treatments to promote cell attachment for adherent cells. To address this limitation, in the past 15 years, materials scientists and bioengineers have launched a series of revolutionary studies in an endeavor to create spatially and temporally controlled extracellular biochemical cues using micro/nanoengineered synthetic instructive and responsive biomaterials and methods. So far, a gallery of techniques has been developed to create and regulate micro/nanoscale patterns of ECM biochemical cues both on 2D substrates and in 3D matrices. These techniques have helped reveal how the spatial organization of ECM biochemical cues and its dynamic regulation can determine cellular behaviors via dynamic cell-ECM and cell-cell interactions. These studies have also provided insights on physiological and pathological functions of biochemical properties of the ECM in tissue morphogenesis, wound healing, and cancer metastasis.

Creating Micro/nanoscale Patterns of ECM Proteins on 2D Substrates. Microcontact printing $(\mu \mathrm{CP})$, derived from soft lithography about 15 years ago, is the most popular method for creating micro/nanocale regular adhesive ECM patterns on 2D substrates. ${ }^{[38,167]}$ In $\mu \mathrm{CP}$, a stamp made in some elastomeric materials (normally PDMS) by replica molding that have micro/ nanoscale relief patterns on its surface are used to transfer thiol-containing molecules or adhesive proteins from the stamp to $2 \mathrm{D}$ substrates through conformal contact (Figure 7a,b). After creation of the stamp, the first step in $\mu \mathrm{CP}$ is to incubate ("ink") the stamp with solutions of thiol-containing molecules (such as alkanethiolates) or adhesive proteins directly. Thiol-containing molecules and proteins can spontaneously adsorb to the stamp 


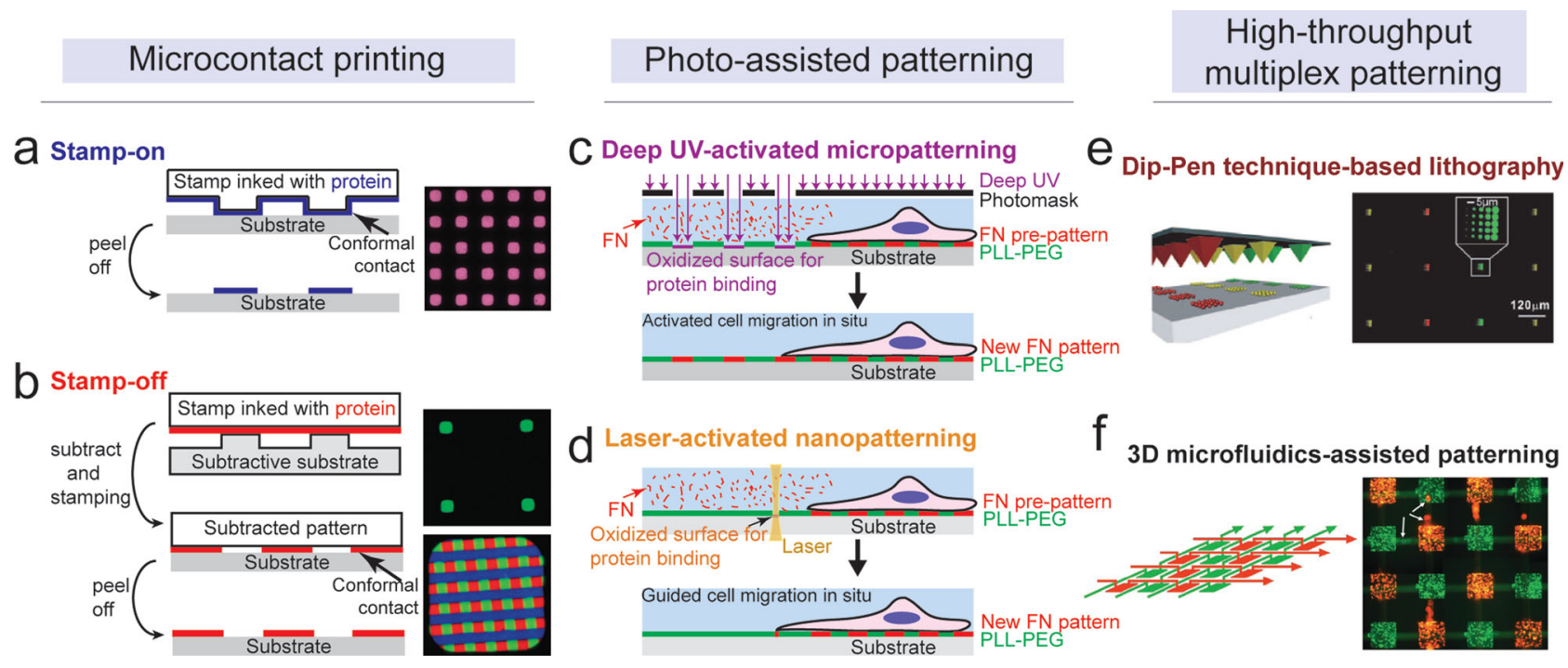

Figure 7. Generating micro/nano-patterns of cell adhesive cues on 2D substrates. (a) (Left) Schematic of conventional ("stamp-on") microcontact printing $(\mu \mathrm{CP}) \cdot{ }^{[38]}$ A PDMS stamp inked with proteins was put into conformal contact with activated substrate and transferred protein patterns after peeling the stamp off. (Right) Fluorescent image of BSA patterns generated by stamp-on $\mu$ CP. (b) (Left) Schematic of subtractive ("stamp-off") $\mu C P .{ }^{[170]} \mathrm{A}$ PDMS stamp uniformly inked with proteins was first subtracted by a micropatterned substrate, and then the subtracted protein pattern was transferred onto the target substrate using conventional $\mu \mathrm{CP}$. (Right) Fluorescent images showing the efficacy of stamp-off $\mu \mathrm{CP}$ in generating distantly separated patterns and multiplexed patterns, respectively. ${ }^{170]}$ Reproduced with permission. ${ }^{[170]}$ Copyright 2011, Royal Society of Chemistry. (c) Schematic of deep UV-activated micropatterning. ${ }^{[4]}$ Deep UV destroyed the cell-repellent PLL-g-PEG coating and oxidized the substrate surface underneath for further binding with solute fibronectin (FN) molecules, thus transferring micro-patterns from the photomask to the substrate, even in the presence of cells. (d) Schematic of laser-activated nanopatterning. ${ }^{[175]}$ Focused laser was used to remove cell-repellent PLL-g-PEG coating and oxidize the substrate within a nano-scale area, thus enabling subsequent binding of FN and nano-patterning of substrate in a maskless fashion and in the presence of cells. (e) Schematic (left) and fluorescent image (right) of using polymer-pen lithography to achieve multiplex protein patterning. [180] Reproduced with permission. ${ }^{180]}$ Copyright 2009, Wiley-VCH (Germany). (f) Schematic (left) and fluorescent image (right) of using a 3D microfluidic system to achieve parallel multiplex protein patterning. ${ }^{[183]}$ Reproduced with permission. ${ }^{[183]}$ Copyright 2000, United States National Academy of Sciences.

surface owing to hydrophobic interactions. To transfer thiolcontaining molecules and adhesive proteins from the stamp to 2D substrates, after the stamp is dried, the stamp is brought into physical contact with 2D substrates that are either chemically reactive (like a thin gold film for thiol-containing ink molecules) $^{[38]}$ or physicochemically prepared (like a PDMS surface treated with UV-Ozone). ${ }^{[108]}$ Thiol-containing molecules and adhesive proteins are then area-selectively transferred to $2 \mathrm{D}$ surfaces based on the relief features on the stamp. For $\mu \mathrm{CP}$ with thiol-containing molecules, the noble metal atoms within the substrate selectively react with thiol groups and thus generate a dense and crystalline/semi-crystalline array of printed molecules, i.e., a self-assembled monolayer (SAM), in order to minimize the surface energy. ${ }^{[168]}$ The terminal group of the alkane chain in the molecules can be tailored, so that patterned areas with thiol-containing molecules on the gold-coated 2D surface become either terminated with cell adhesive islands or highly hydrophobic to allow adhesive ECM molecules to spontaneously adsorb onto such hydrophobic surfaces from aqueous solutions. Remaining regions on the 2D surfaces patterned with thiol-containing molecules and adhesive proteins can be rendered inert to protein adsorption using commercially available PEG-based surfactants and polymers. So far, $\mu \mathrm{CP}$ has allowed patterned printing of ECM ligands of interest onto PDMS, glass, or polystyrene surfaces. ${ }^{[8,38]}$

Conventional $\mu \mathrm{CP}$, which is "stamp-on" in nature, uses protruding micro/nanoscale features on the stamp to directly print adhesive protein patterns on 2D surfaces (Figure 7a). However, it is difficult for "stamp-on" $\mu \mathrm{CP}$ to print distantly separated (due to roof collapse of elastomeric stamps) or very small protein patterns (due to buckling of fine stamp features). ${ }^{[38]}$ Recently, an alternative version of $\mu \mathrm{CP}$, called "stamp-off", was developed to address the limitations of "stamp-on" $\mu \mathrm{CP}$ (Figure 7b). ${ }^{[169,170]}$ The "stamp-off" $\mu$ CP first applies a subtractive PDMS stamp containing negative patterns of interest to remove undesired ink proteins from a flat featureless PDMS stamp that is originally uniformly coated with ink proteins. The flat featureless PDMS stamp containing desired patterns of adhesive proteins is then brought into conformal contact with a target 2D substrate to finalize the protein transfer process, just as is done in conventional $\mu \mathrm{CP}$. The intermediate subtractive step in "stamp-off" $\mu$ CP can be repeated so that different ECM proteins can be coated in regular patterns on the flat featureless PDMS stamp. Moreover, since in "stamp-off" $\mu \mathrm{CP}$, the subtractive PDMS stamp to remove adsorbed ECM proteins contains identical but inverse features, it is rather easy for the "stampoff" $\mu \mathrm{CP}$ to achieve high-fidelity printing of ECM proteins down to a resolution of $100 \mathrm{~nm}$.

Since its invention, $\mu \mathrm{CP}$ has proven to be a versatile and powerful tool to create micro/nanopatterns of ECM proteins on 2D substrates. However, it is worth noting that as strong hydrophobic interactions between proteins and PDMS surfaces can result in a requirement for a strong peeling force to remove the PDMS stamp from the target 2D substrate, this peeling force can sometimes be strong enough to break or rupture the surface of soft materials, including soft hydrogels and PDMS. 
Thus, it requires caution and careful adjustments of experimental parameters to modulate interfacial mechanics in contact printing to achieve high-quality, non-destructive and complete transfer of proteins in $\mu \mathrm{CP}$ on soft materials. ${ }^{[171]}$ Moreover, the photo-assisted patterning, which we will discuss later, is another option for such a task. ${ }^{[172]}$

An alternative method to generate regular micropatterns is stencil-assisted micropatterning, which was developed about a decade ago. ${ }^{[173]}$ A stencil is usually a thin film of soft materials (e.g., PDMS) with arrays of microscale through-holes within it, and it self-seals with the target substrate when they are brought into contact before applying protein solution (or cell culture medium) and seeding cells. Therefore, the substrate underneath the stencil was protected against protein adsorption and subsequent cell seeding, while only the area within the holes were exposed to cells and culture environment. Moreover, after peeling off the stencil and generating the primary micropatterns, one might also adsorb secondary cell adhesive proteins onto the originally protected area and thus create a secondary micropattern for studying either the combinatorial effects of different adhesion ligands or microstructured coculture of different types of cells.

Microcontact printing and stencil-assisted micropatterning both require access to microfabrication for generating patterned stamps or stencil masks, limiting their usage for labs that have no access to microfabrication. To address this, Théry and colleagues adapted the idea of photolithography and developed deep UV-activated micropatterning (photomask required) (Figure 7c) and laser-activated nanopatterning (maskless) (Figure 7d) techniques to create stable as well as dynamic ECM adhesion patterns on 2D surface with a sub-micron resolution. ${ }^{[41,174-176]}$ In these two methods, deep UV exposure (with a wave length $<200 \mathrm{~nm}$ ) with photomasks and focused laser first locally oxidize cell repellent polymer coating (e.g., poly(Llysine)-g-poly(ethylene glycol), PLL-PEG) on target cell culture substrates (e.g., glass or PS) to expose surfaces underneath the coating. Exposed surface areas are hydrophilic and provide sites to covalently bind to ECM proteins from aqueous solutions. These two photo-assisted ECM patterning techniques, in principle, can define and modify ECM adhesive patterns in the presence of cells in real time, opening the door for biological assays studying cellular behaviors in response to dynamic modifications of cell adhesion patterns. Operations of deep UVactivated micropatterning and laser-activated nanopatterning depend critically on aqueous solutions, and they are compatible with most biological applications to dynamically engineer spatially patterned ECM adhesive cues.

Dip-pen lithography and it various derivatives, which are capable of rapid, high-throughput printing of ECM biochemical cues at a resolution down to $<10 \mathrm{~nm}$, are often used for highthroughput screening of effects of ECM biochemical cues and their combinations on cellular behaviors (Figure 7e). ${ }^{[177-181]}$ Equipped with a regular array of scanning probes ("pens"), dip-pen lithography and it derivatives first "dip" the "pens" into protein inks before placing the pens onto prescribed locations on a 2D surface to transfer proteins from the pens onto the substrate via meniscus. As highly automated commercially available tools, dip-pen lithography and its derivatives (dip-pen lithography, ${ }^{[177]}$ polymer pen lithography, ${ }^{[179,180]}$ and scanning probe block copolymer lithography ${ }^{[181]}$ in conjunction with different designs of pens and inks ${ }^{[178]}$ have delivered a great power to achieve large-scale high-throughput patterning of ECM proteins with a sub-micron resolution.

There are other reported methods such as 2D and 3D microfluidics-assisted patterning ${ }^{[182,183]}$ (Figure 7f), microarray spotting ${ }^{[10,184,185]}$ and electrohydrodynamic jet (E-Jet) printing ${ }^{[186-188]}$ that were developed in the last decade for large-scale parallel patterning of different ECM proteins down to a micron scale. In light of the diverse applications of microfluidics for biomedical research, microfluidics-assisted patterning can potentially be integrated with other microfluidic cell culture and analysis components on the same chip for integrated high-throughput cell-based assays. In contrast to the top-down micro/nanoengineering approaches discussed above, nanoscale patterning of ECM proteins can also be achieved in a bottom-up manner, for example, by using block copolymer self-assembly. ${ }^{[188-192]}$

Dynamic Regulation of ECM Patterns on 2D Substrates. Dynamic cellular responses to changes in microenvironmental cues are a fundamental property of living systems. However, designs of ECM patterns in conventional surface micropatterning tools such as $\mu \mathrm{CP}$ and dip-pen lithography are fixed at the point of fabrication. Hence, cellular responses to ECM patterns generated using such tools can only be observed at a steady state. This has been a major limitation for experimental studies where dynamic cellular responses to changes in microenvironmental cues are desired. In recent years, developments of dynamical microscale ECM patterns on 2D substrates have enabled better mimicking of dynamic ECM remodeling. Four different strategies that use non-toxic stimulations on demand and are easy to implement, including voltage-driven dynamic switch of micropatterns, photo-assisted dynamic micropatterning, micromechanical reconfigurable cell coculture, and click chemistry-based dynamic micropatterning, have been successfully exploited to achieve dynamic coating of ECM patterns.

For voltage-driven dynamic switch of micropatterns (Figure 8a), the key lies in synthesis of electroactive polymers, which either contain voltage-cleavable cell adhesion peptides (e.g., RGD) grafted over a cell repellent backbone ${ }^{[193,194]}$ or have cell repellent chains containing an electroactive functional group that under oxidization by voltage, can bind to adhesive ligands from aqueous solutions. ${ }^{[193,195,196]}$ Initial micropatterns of electroactive polymers can be achieved using either $\mu \mathrm{CP}^{[195]}$ or stencil-assisted micropatterning ${ }^{[194,197]}$ on 2D substrates precoated with electrodes (Figure $8 \mathrm{~b}$ ). A change of voltage in electrodes can either cleave the RGD peptides from the cell repellent backbones grafted on the electrode, thus releasing attached cells (Figure 8c), ${ }^{[193,194]}$ or oxidize the electroactive functional groups so that they can bind to adhesive ligands from aqueous solutions to render originally inert electrode surfaces become adhesive to trigger new cell adhesions (Figure 8d). ${ }^{[193,195,196]}$ An alternative method reported by Jiang and colleagues utilized the phenomenon of electrochemically desorption of self-assembled monolayers (SAMs) on gold electrodes and thus releasing adhesive proteins originally absorbed on the SAMs. ${ }^{[198,199]}$

The deep UV/laser-assisted micro/nanopatterning methods discussed in the last section were good examples of using local light exposure to oxidize cell repellent polymer coating so that exposed surfaces underneath the coating can absorb 


\section{Voltage-driven}

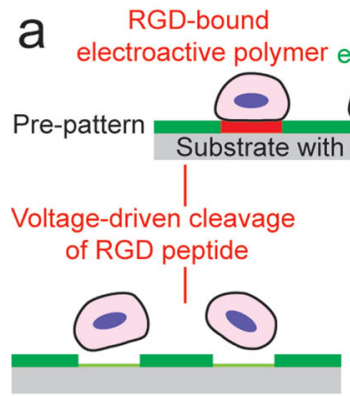

Releasing attached cells

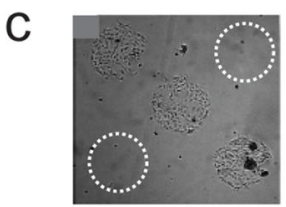

Protein resistant polyme

$$
0
$$

electrode

(4)

Voltage-driven oxidization for binding new RGD peptide

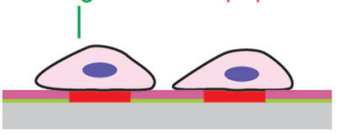

Releasing cell area restraint

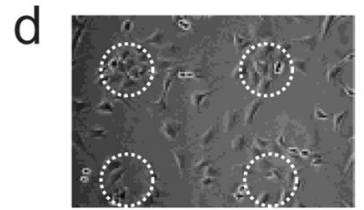

Light-driven
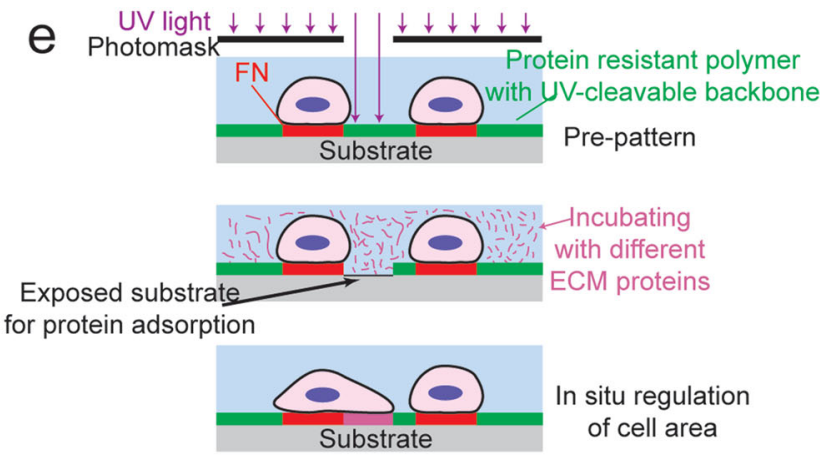

f
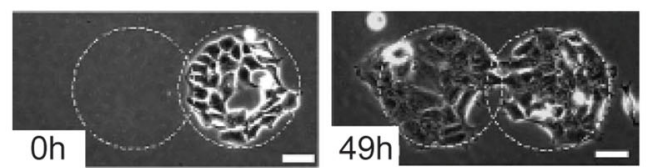

Mechanical-driven

g

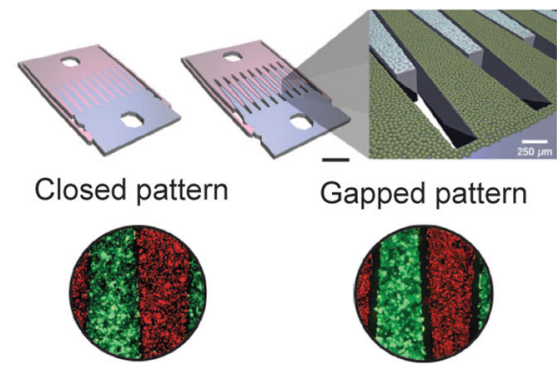

Click chemistry-driven

h

APP cell RGD-modified APP layer

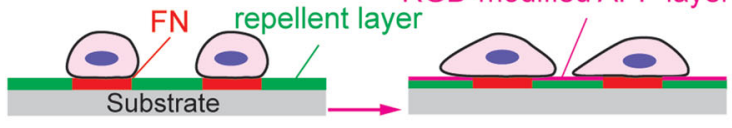

Adding BCN-RGD to culture medium

Figure 8. Dynamic regulation of micro/nano-patterns of cell adhesive cues on 2D substrates. (a) Schematic of using electroactive polymers for voltagedriven dynamic regulation of micro-patterns. See text for detailed description. (b) Phase contrast image of pre-patterned cell clusters. ${ }^{[193]}$ Reproduced with permission. ${ }^{[193]}$ Copyright 2003, American Chemical Society. (c) Phase contrast image of cell clusters remained after voltage-driven releasing of two other clusters (released clusters were marked by white circles). ${ }^{[194]}$ Reproduced with permission. ${ }^{[194]}$ Copyright 2006, American Chemical Society. (d) Phase contrast image of cells spreading out after the area outside the original patterns (marked by white circles) were activated by voltage and functionalized with solvent RGD peptides. ${ }^{[193]}$ Reproduced with permission. ${ }^{[193]}$ Copyright 2003, American Chemical Society. (e) Schematic of lightdriven cleavage of cell-repellent polymer coating, rendering in situ modulation of cell adhesion patterns. ${ }^{[202]}$ ( $f$ ) Phase contrast images showing guided cell migration after releasing neighboring (left circle) cell-repellent coating in situ. Reproduced with permission. ${ }^{[202]}$ Copyright 2006, Elsevier. (g) Schematic (top) and fluorescent images (bottom) of mechanical-driven modulation of hepatocytes-stromal cells co-culture patterns. ${ }^{[203]}$ Reproduced with permission. ${ }^{[203]}$ Copyright 2007, United States National Academy of Sciences. (h) Schematic of one-step dynamic regulation of micro-patterns by click chemistry. ${ }^{[204]}$ Solute BCN-RGD reacted with and immobilized onto APP cell-repellent coating and relieved the original restraints on cell adhesion.

ECM proteins from aqueous solutions and become adhesive (Figure 7c,d). Alternatively, photo-assisted micropatterning can be achieved by initially patterning cell repellent polymers containing UV-cleavable backbones on surfaces, which, under a local UV light exposure (through either a photomask or using focused lasers), can release cell repellent groups and thus render the exposed areas available for absorption of adhesive molecules from aqueous solutions (Figure 8e,f). ${ }^{[200-202]}$ Multiplexed dynamic micropatterning can be achieved with these light-driven strategies by using multiple photomasks and sequential light exposures. ${ }^{[201]}$
To dynamically regulate cell-cell communications in a cell coculture system, Hui and Bhatia developed an array of micromachined plates, or the so called micromechanical reconfigurable culture ( $\mu \mathrm{RC})$, that could be physically rearranged to change the spatial organization and composition of the coculture (Figure $8 \mathrm{~g}$ ). ${ }^{[203]}$ Specifically, the $\mu \mathrm{RC}$ device consisted of two parts with interlocking comb fingers and an integrated snap-lock mechanism. The parts could be fully separated, locked together with the fingers in contact, or locked together with a fixed gap between the comb fingers. Using the $\mu \mathrm{RC}$, Hui and Bhatia were able to demonstrate dynamic regulations 
of direct cell-cell interactions and cytokine-mediated soluble signaling between hepatocytes and supportive stromal cells and their effects on hepatocellular phenotype.

Very recently, van Dongen and colleagues developed a rapid and easy-to-implement method to dynamically control cell adhesion patterns via click chemistry (Figure 8h). ${ }^{[204]}$ The initial adhesive micropatterns were generated using azido(PLL-g-PEG) (APP) as cell repellent molecules coated between fibronectin (FN) adhesive islands. Using click chemistry, APP allowed spontaneous and catalyst-free cycloaddition to immobilize adhesive molecules of choice when the adhesive molecules were paired with compounds containing the strained cyclooctyne bicyclo[6.1.0]-nonyne (BCN). Thus, APP could immobilized BCN-RGD peptides from aqueous solutions by binding to $\mathrm{BCN}$ and thus abrogated the cell repellence property and rendered the entire surface accessible for cell adhesion.

3D Biochemical Patterning of Hydrogels. Despite significant advances of synthetic hydrogels, engineering biophysical and biochemical cues in 3D cell culture using synthetic hydrogels remains a complex problem with considerable challenges to overcome in the pursuit of complete understanding of dynamic cellular behavior in 3D environment. Recently, several groups have made exciting progress using bio-active and -responsive hydrogels to create dynamic microenvironment in demand for 3D cell culture. These highly tunable materials have provided bioengineers and biological scientists with new ways not only to treat patients in the clinic but to study fundamental cellular responses to dynamic 3D ECM microenvironment as well. ${ }^{[132-135,205-208]}$

In recent decade, several mechanisms have been demonstrated capable of regulating the mechanical and biochemical properties of $3 \mathrm{D}$ hydrogels with high specificity. ${ }^{[42,45]}$ So far, the most powerful and versatile method that spatiotemporally modulates cell adhesive cues within 3D hydrogels is established upon cytocompatible photo-chemistries, which incorporates photoactive linkers into the hydrogel and uses photopolymerization/cleavage to achieve dynamic biomechanical and biochemical remodeling of the 3D hydrogel (Figure 9).

In recent years, the West group exploited the photo-chemistry between PEG-diacrylate (PEGDA) hydrogel and acrylatederivatized cell adhesive moieties (e.g., acryl-PEG-RGDS) in their development of a UV-tunable 3D hydrogel. After the initial radical chain photo-polymerization, which forms the PEGDA hydrogel, the acryl-PEG-RGDS was perfused into the gel and incorporated into particular regions illuminated by UV light using either a photomask or two-photon absorption laser scanning lithography (TPA-LSL) (Figure 9a). ${ }^{[205,206]}$ In addition to such biochemical micropatterning, if the acrylatederivatized moieties were designed as a crosslinker, which contains acryl groups on both ends, rather than one adhesive ligand on one end and one acryl group on the other, the same photo-chemistry as described above could achieve dynamic biomechanical micropattering of the hydrogel via remodeling local crosslinking properties (Figure 9a).

Recently, the Shoichet group incorporated photocaged thiols into an agarose hydrogel that enables multiplex 3D micropatterning of soluble factors. When exposed to two-photo irradiation, the thiols were uncaged and reacted with and subsequently immobilized maleimide peptides which were grafted with bio-active moieties and present in the solvent. By using maleimide peptides functionalized with barnase and streptavidin, respective, they sequentially created micropatterns of binding sites for immobilizing two soluble stem cell differentiation factors (sonic hedgehog ( $\mathrm{SHH}$ ) and ciliary neurotrophic factor (CNTF)), which were grafted onto barstar and biotin, respectively, and achieved simultaneous multiplex micropatterning in 3D hydrogels (Figure 9a,b). ${ }^{[207]}$

As discussed previously, the Anseth group developed photodegradable PEG-based hydrogels through rapid UV-polymerization of cytocompatible macromers for remote manipulation of gel properties in situ. ${ }^{[132-135]}$ Such a technique enables reversible biochemical micropatterning in 3D hydrogels (Figure 9a,c). As a base material, the hydrogel was fabricated by exploiting a strain promoted, azide-alkyne cycloaddition (SPAAC) reaction between a PEG macromer and photoactive peptides that contain alkenes, which could photo-chemically react to thiol groups under light exposure. As the elements for biochemical functionalization, molecules containing the bio-active moieties of interest were coupled to both a thiol group (for initially photo-coupling the bio-active moieties to the hydrogel) and a photolabile o-nitrobenzyl moiety that enables photo-cleavage

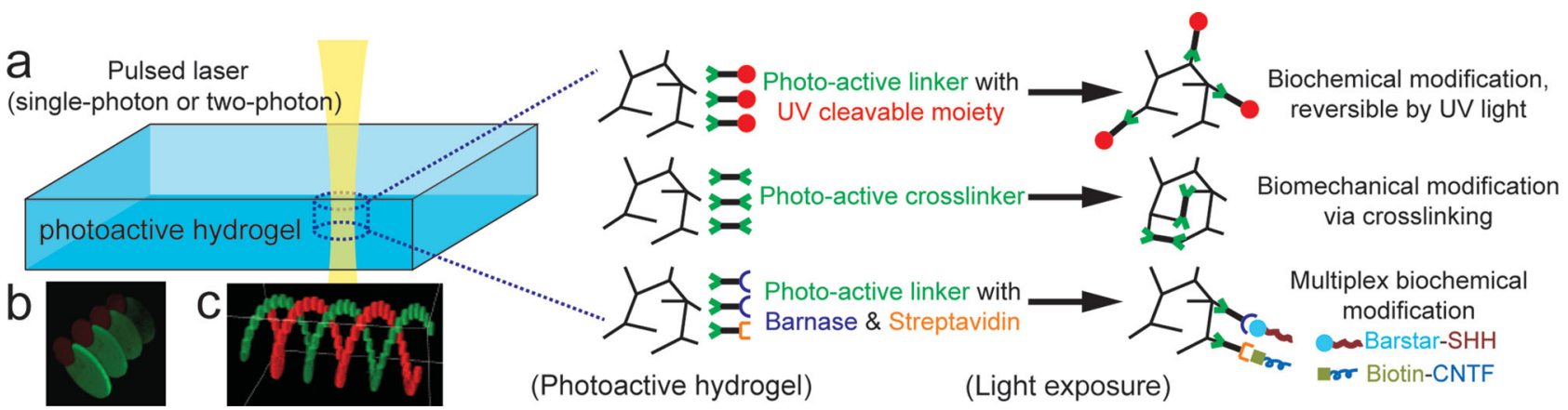

Figure 9. Micro/nano-patterning of biochemical and biophysical cues in 3D hydrogels. (a) Schematic of micro/nano-patterning in 3D hydrogels using focused laser (single-photon or two-photon). Using different photo-chemical designs of crosslinkers, one could achieve reversible biochemical patterning, mechanical modulation and multiplex immobilization of biochemical cues (see text for detailed description). ${ }^{[135,206,207]}$ (b) Fluorescent confocal micrograph of 3D micro-patterns of SHH (green) and CNTF (red) within an agarose hydrogel. [207] Reproduced with permission. [207] Copyright 2011, Nature Publishing Group. (c) Fluorescent confocal micrograph of micro-patterned 3D helices of fluorescently labeled peptides, of which one (red) was reversibly removed after UV exposure. ${ }^{[135]}$ Reproduced with permission. ${ }^{[135]}$ Copyright 2012, Wiley-VCH (Germany). 
afterwards. The initial biochemical micropatterining of the hydrogel is achieved via thiol-ene reaction under visible light and the subsequent selective removal of those biochemical cues is made by UV exposure through a photomask. Similar designs, which incorporated photolabile moieties in the crosslinkers within the gel, also enabled hydrogel degradation for modulating 3D gel mechanics and cell movements. ${ }^{[135]}$

Initially, single-photon absorption (SPA) photolithography was applied to synthesize these photo-chemically modulated hydrogels, for the sake of fast processing speed and economy. However, SPA photolithography is difficult to achieve fully encapsulated 3D micropatterning since the light goes through the whole sample. In recent years, TPA photolithography is used more often and becomes popular due to its advantage of small excitation volume (roughly half the excitation wavelength), making it competent for complex, high-resolution free form 3D micropatterning of the biomechanical and biochemical properties within the hydrogel.

Effects of Patterned Adhesive Cues on Cellular Behaviors. The last 15 years have witnessed a rapid advance of the capability to spatially pattern ECM biochemical cues at molecular, cellular, and tissue scales in both 2D and 3D microenvironments, critical for revealing biological functions of cell-ECM adhesive interactions in regulating mechano-sensitive and -responsive cellular behaviors. A notable example patterned regular adhesive ECM islands on 2D surfaces using $\mu \mathrm{CP}$ to study the effect of cell spreading area on cell apoptosis and proliferation. ${ }^{[167]}$ This study revealed that severe cell confinement resulting in small cell spreading area promoted cell apoptosis while cell spreading without much confinement (leading to large cell spreading area) supported cell growth and proliferation. ${ }^{[167]}$ In another study, by using $\mu \mathrm{CP}$ to coat flat PDMS surfaces with distinct patterns of adhesive ECM islands, McBeath and colleagues reported that in response to a bipotential differentiation medium that contained inducers for both adipogenic and osteogenic differentiations, single hMSCs confined to small ECM islands selectively underwent adipogenesis, whereas single hMSCs on large ECM islands were biased toward osteogenesis. ${ }^{[15,209]}$ Using 2D and 3D adhesive patterning tools, cell shape and curvature as well as relative cell location in a cluster of cells were also recently identified to regulate lineage specifications of hMSCs. ${ }^{[160,210,211]}$ Specifically, Ruiz and Chen applied microscale patterning approaches to control geometries of both 2D and 3D multicellular structures of hMSCs. The authors reported that in the presence of soluble factors permitting both osteogenic and adipogenic differentiations, hMSCs at the edge of multicellular structures selectively differentiated into the osteogenic lineage, whereas those in the center became adipocytes. ${ }^{[210]}$ Kilian and colleagues demonstrated that in response to a bipotential differentiation medium that contained inducers for both adipogenic and osteogenic differentiations, single hMSCs cultured in rectangles with increasing aspect ratio and in shapes with pentagonal symmetry but with different subcellular curvature - and with each occupying the same area displayed different adipogenesis and osteogenesis profiles. ${ }^{[211]}$ Recently, using single cell micropatterning on soft PA gel substrates, Lee and colleagues further demontrated that cell shape was a key regulator that could switch hMSCs to undergo either the adipogenic (round shape) or the neuronal (elongated shape) differentiations when subject to compliant substrate mechanics. ${ }^{[160]}$ All together, these studies provided compelling evidence supporting that cell shape and geometry are key regulators of cell functions when using experimental strategies to pattern the spreading and morphology of adherent cells.

Our mechanistic understanding of effects of cell size, shape, and geometry in regulating mechanosensitive cellular behaviors remains incomplete. Existing experimental evidence support that mechanosensitive cellular behaviors are mediated by a combination of soluble factors and insoluble adhesive as well as mechanical signals in the local cell microenvironment, which are transmitted through interplay between adhesion signaling and actin cytoskeleton to intracellular space to integrate with intracellular signaling pathways for regulation of long-term cell functions.

While microscopic patterns of adhesive cues facilitated studies at the cellular and tissue scales, nanoscale control and patterning of biochemical signals provided platforms to study the cell-ECM adhesive interactions down to a molecular level. ${ }^{[148]}$ As reported by Arnold and colleagues, ${ }^{[212]}$ by using block-copolymer micelle nanolithography to pattern gold nanodots coated with adhesive peptides, when the spacing between these nanodots exceeded approximately $70 \mathrm{~nm}$, cell adhesion and spreading, FA, and actin stress fiber formations were significantly impaired, likely owing to the restricted clustering of integrin molecules by the distance between the adjacent gold nanodots. ${ }^{[212-214]}$ In another relevant study using nanoimprint lithography to pattern gold nanodots functionalized with binding ligand RGD, Schvartzman and colleagues reported a drastic increase in the spreading efficiency of cells on arrays of different geometric arrangements of the nanodots when at least four liganded sites were spaced no more than $60 \mathrm{~nm}$ apart, with no dependence on global density. ${ }^{[215]}$ This interesting observation pointed to the existence of a minimum of four integrin adhesion units required for initial growth and maturation of nascent FAs on fibronectin as defined in space and stoichiometry. In another recent study, Coyer and colleagues applied nanoimprint lithography to pattern 2D nanoscale adhesive islands within a cell adhesion-resistant background to study the threshold area of ECM ligand required for stable FA assembly and CSK contractile force transduction. ${ }^{[216]}$ The authors observed that integrin clustering and adhesive force were strongly modulated by the geometry of the nanoscale adhesive area. Interestingly, individual adhesive nanoisland area, not the number of nanoislands or total adhesive area, controlled integrin clustering and adhesion strength. Importantly, the authors discovered that below an area threshold $\left(0.11 \mu \mathrm{m}^{2}\right)$, very few integrin clusters and negligible adhesive forces were generated.

Besides regulating cell shape and geometry of adherent cells as well as controlling nanoscale molecular organization of FAs, micro/nanoscale patterning of adhesive ECM cues also produced a set of 2D functional surfaces to modulate intracellular actin CSK organization. Developed by Théry and colleagues using $\mu \mathrm{CP}$, these functional surfaces helped reveal that the spatial arrangement of ECM patterns could play an important role in determining intracellular polarity, mitotic spindle orientation, and post-cytokinesis cell-cell junction positioning, implicating a fundamental role of cell-ECM interactions in modulating intracellular actin CSK organization to mediate cell division and tissue morphogenesis. ${ }^{[217-221]}$ 
All together, these studies using well-controlled cell-ECM interactions at the microscale and nanoscale have demonstrated the molecular sensitivity and dynamic organization of FAs, which are regulated by local force-mediated equilibrium between pathways controlling cell adhesion, actin cytoskeleton contraction, and the structural linkage of cell adhesion that transmits the forces, allow the force balance to be tipped by factors that regulate these biomechanical parameters. These studies have also implicated functional interplay between cellECM interactions (and its downstream adhesion signaling) and intracellular actin CSK organization, critical for cell division and tissue morphogenesis.

\subsection{Micro/nanoengineered Functional Biomaterials to Control and Monitor Cell-ECM and Cell-Cell Interactions}

In vivo, adherent cells attach and pull the surrounding ECM or adjacent cells they adhere to, utilizing the intracellular myosin II-based actin CSK contractile machinery. ${ }^{[2,19,222]}$ In the last decade, such a direct physical interaction between cells and the cell microenvironment has been shown critically important for cellular sensing of substrate rigidity via adhesion-mediated intracellular signaling. ${ }^{[19,127]}$ In recent years, cumulative evidence has further demonstrated that cellular contractile forces against the surrounding ECM hold a great significance not only for mediating long-term mechanoresponsive cellular behaviors such as stem cell differentiation, ${ }^{[15,24,26,131,223]}$ tissue morphogenesis ${ }^{[2]}$ and cancer metastasis, ${ }^{[17,18,224]}$ but also for regulating dynamics and morphogenesis of force-transmitting cellular structures such as FAs, ${ }^{[8,225,226]}$ adherence junctions $(\mathrm{AJs})^{[227,228]}$ and ion channels, ${ }^{[229]}$ which are involved in a range of force-dependent functions and known to relay extracellular biomechanical signals to intracellular signaling cascades. Thus, understanding mechanical force transmission between adherent cells and the local ECM microenvironment is vital for appreciating the critical role of mechanical forces in fundamental cell and developmental biology and revealing molecular and cellular mechanisms underlying mechanotransduction and mechanoresponsive cellular behaviors.

Studying force transmission between adherent cells and the local cell microenvironment necessitates the development of tools and methods to directly measure such mechanical forces. Since mechanical force (or stress) is not a quantity that can be measured directly, one needs to quantify in some way the deformation (or strain) of the local cell microenvironment (or intracellular components) under the influence of extracellular (or intracellular) force applications, in order to convert such deformation (or strain) to extracellular (or intracellular) mechanical forces (or stress). In the last decade or so, many novel micro/ nanoengineered extracellular and intracellular deformation (or strain) sensors have been developed, in conjunction with the advance of high-resolution imaging technologies, to control and monitor cell-ECM and cell-cell mechanical interactions as well as intracellular forces at the molecular, cellular and tissue levels in both 2D and 3D contexts.

Compliant Hydrogels Embedded with Fiduciary Markers. A most straightforward way to visualize the deformation within a continuum is to use labeled micro/nanoparticles embedded in the continuum as fiduciary markers to quantify their relative displacements under the influence of force applications. The traction force microscopy (TFM), originally developed by Dembo and Wang, ${ }^{[230]}$ is the best example that follows this principle to measure cellular contractile forces against a compliant hydrogel surface. Since its invention, TFM has been greatly improved by different research groups. ${ }^{[131,223,228,231-238]}$ So far, TFM has become the most adapted force measurement technique to study cell-ECM mechanical interactions owing to its simple principle and the convenience of generating compliant hydrogels embedded with micro/nanoscale fluorescence beads.

In TFM, submicron or nanometer-sized fluorescent beads that serve as fiduciary markers are first mixed with hydrogel precursor solution before the gel is subjected to polymerization and then functionalized with adhesive molecules for cell seeding and attachment. Positions of fluorescent beads close to the hydrogel top surface are recorded using high-resolution fluorescence microscopy both when cells are attached on the hydrogel top surface and after the cells are lysed or re-suspended from the surface (Figure 10a). In-plane displacement field for the hydrogel surface is then obtained by registering fluorescent images of beads with the cells attached on the hydrogel surface against the one without cells (Figure 10a, middle panel). With the in-plane hydrogel displacement field obtained as well as the elastic modulus of the hydrogel known, reconstitution of traction force (stress) applied by the cells on the hydrogel top surface (Figure 10a, middle panel) can be achieved by solving a theoretical reverse problem of a half-space elastic solid subjected to shear (in-plane) tractions using a Green's function from the Boussinesq's theory. ${ }^{[230]}$ It should be noted, though, that the mathematical nature of such a reverse problem dictates that the reconstituted traction force field is sensitive to small variations of the hydrogel surface displacement field. Thus, TFM has a stringent requirement for the resolution and precision of fluorescent bead positions at the hydrogel top surface recorded by fluorescence microscopy. ${ }^{[232,237]}$ Further, certain forms of regularization may be necessary in TFM to reconstitute reasonable traction force fields. ${ }^{[231,237]}$ A recent study improved the accuracy of TFM by extracting the hydrogel displacement field using two sets of nanoscale beads functionalized with different fluorescent "colors" in conjunction with using an improved imaging processing technique. ${ }^{[232]}$ Some other recent studies also advanced the traction force reconstitution algorithm to improve the resolution of TFM. ${ }^{[232,233,237]}$

Instead of using fluorescent micro/nano-beads as fiduciary markers distributed throughout the whole substrate, Balaban and colleagues adapted soft lithography and patterned shallow microscale islands of fluorescent photoresist right underneath the substrate surface. Since the undeformed grid of the pattern is known a priori, it enabled real-time readout of substrate deformation by just registering the image of deformed pattern with cells on against the known undeformed pattern predetermined by design. Such a transparent substrate is also compatible with high-resolution live-cell imaging. Using this modified version of TFM, they revealed the force-dependence of FA assembly for the first time. ${ }^{[225]}$

Even though powerful and convenient for quantifying tangential traction forces on the hydrogel top surface, conventional 2D TFM does not provide information regarding gel 


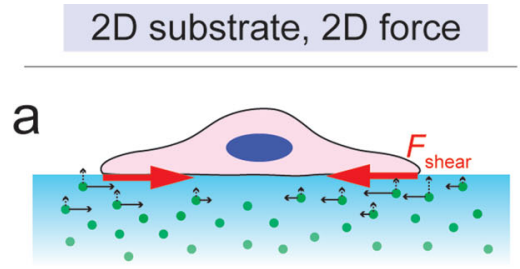

Top view

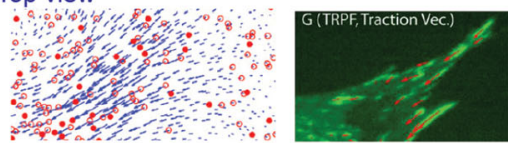

Beads displacement field Reconstituted force field

Cell-cell force
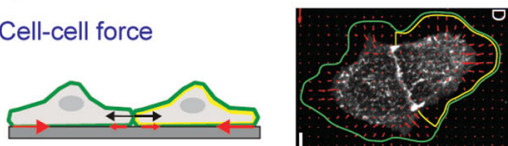

b

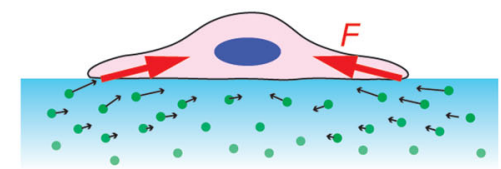

Beads displacement field

Top view

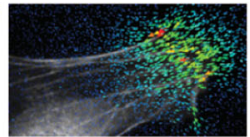

Reconstituted stress field

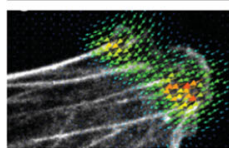

\section{Integrated TFM in fluidic environment}

Endothelium mechanoresponsiveness under shear flow
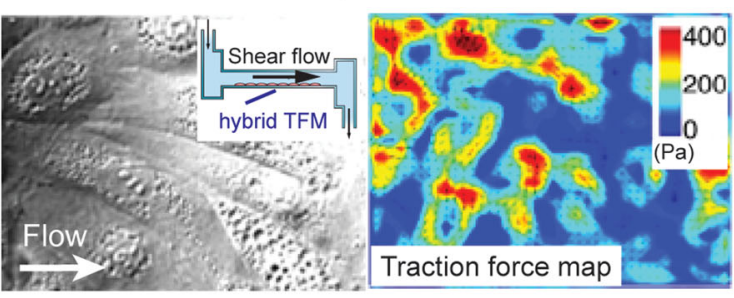

e

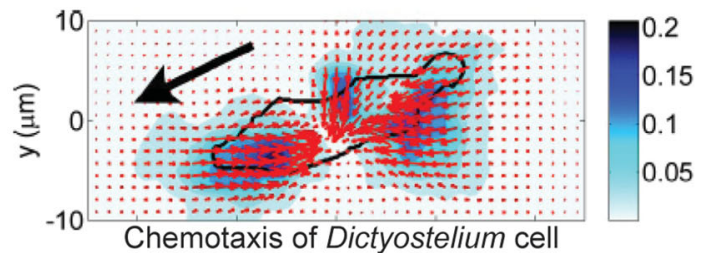

Integrated TFM in dynamic stretch device

f
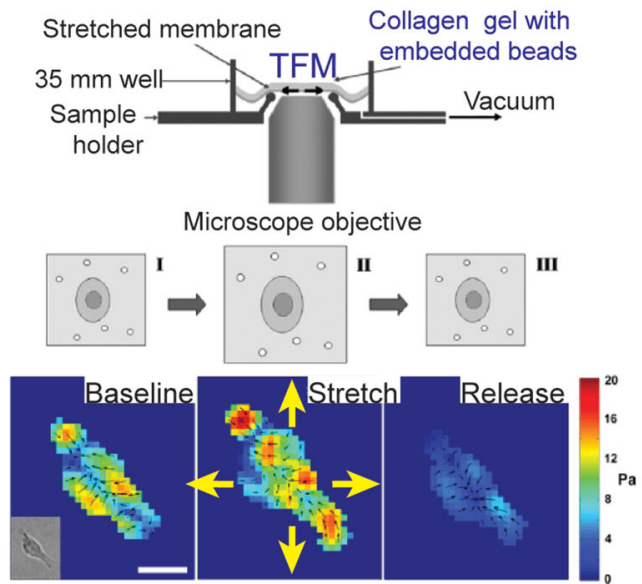

Figure 10. Microengineered traction force microscopy (TFM) in 2D and 3D contexts. (a) (top) Schematic of 2D TFM. Although both in-plane (solid line with arrow head) and out-of-plane (dash line with arrow head) displacement occurred to each bead, only the former was recorded in conventional 2D TFM and only in-plane traction stress was reconstituted. (middle) Extracted beads displacement field and reconstituted traction force field by 2D TFM. ${ }^{[232]}$ Reproduced with permission. ${ }^{[232]}$ Copyright 2008, Cell Press. (bottom) Schematic and fluorescent image showing the application of 2D TFM in characterizing cell-cell force. ${ }^{[228]}$ Reproduced with permission. ${ }^{[228]}$ Copyright 2011, United States National Academy of Sciences. (b) (top) Schematic of hybrid TFM. The 3D displacement of each bead was recorded using confocal microscopy and 3D traction force on 2D substrate surface was reconstituted. ${ }^{[237]}$ (middle) In-plane displacement of beads and reconstituted in-plane traction stress. (bottom) Out-of-plane displacement of beads and reconstituted out-of-plane traction stress. Reproduced with permission. ${ }^{[237]}$ Copyright 2013, United States National Academy of Sciences. (c) (top) Schematic of 3D TFM in hydrogel. (bottom) 3D displacement of beads in gel and reconstituted traction stress on 3D cell-ECM interface. ${ }^{\text {[223] }}$ Reproduced with permission. ${ }^{[223]}$ Copyright 2010, Nature Publishing Group. (d) Phase contrast image (left) and traction force map (right) of a confluent endothelial monolayer under shear flow. Inset: the schematic of a circulatory fluidic channel with integrated gel substrate for performing hybrid TFM. ${ }^{\text {[245] }}$ Reproduced with permission. ${ }^{[245]}$ Copyright 2012, United Stated National Academy of Sciences. (e) Cellular contractile force map of a Dictyostelium cell undergoing chemotaxis. ${ }^{[236]}$ The gradient of chemokine is shown with the black arrow. Reproduced with permission. ${ }^{[236]}$ Copyright 2007, United Stated National Academy of Sciences. (f) Schematic (upper) and traction force maps (bottom) of integrated 2D TFM in a miniaturized cell-stretching device for studying the dynamics of cellular contractility during and after a transient equibiaxial stretch. ${ }^{[247]}$ Reproduced with permission. ${ }^{[247]}$ Copyright 2008, Cell Press.

displacements as well as cellular traction forces perpendicular to the hydrogel surface (Figure 10a). Although the Boussinesq theory predicts negligible coupling between in-plane displacements and out-of-plane forces, i.e., in-plane displacements and in-plane traction forces are self-consistent and thus 2D TFM should give a reasonably correct in-plane force field, ${ }^{[230]}$ there has always been curiosity about cellular traction forces along the third dimension perpendicular to the hydrogel surface. Very recently, different groups successfully developed 3D versions of conventional 2D TFM (hereafter referred to as "hybrid TFM") 
to allow measurements of 3D cell-ECM mechanical interactions on a 2D substrate (Figure 10b). ${ }^{[235,237,239]}$ For example, Legant and colleagues recently applied high-spatiotemporal-resolution confocal microscopy and improved imaging processing and numerical methods to extract 3D displacement fields of a hydrogel underneath migrating or spreading mammalian cells to reconstitute both in-plane and out-of-plane cellular contractile forces exerted on the hydrogel through FAs (Figure 10b, middle and bottom panels). ${ }^{[237]}$ Notably, using this method with mouse embryo fibroblasts (MEFs) expressing EGFP tagged paxillin (a FA protein), Legant and colleagues observed shear traction forces that were centered under elongated FAs whereas upward and downward normal traction forces were detected on distal (toward the cell edge) and proximal (toward the cell body) ends of FAs, respectively, suggesting that these forces produced significant rotational moments about FAs. Since the hydrogel on the proximal side of FAs are compressed by FAs, which can lead to hydrogel surface instability under compression, ${ }^{[102]}$ it calls for caution when applying "hybrid TFM", which still is a reverse method, to calculate high-resolution cellular traction forces that assumes homogeneous material properties as well as non-bifurcating phenomena.

Using a similar strategy, a few groups also recently developed a technique to quantitatively measure 3D cellular contractile forces exerted by cells fully encapsulated in compliant hydrogel matrices (hereafter referred to as "3D TFM") (Figure 10c). ${ }^{[131,223,240,241]}$ Although developing 3D TFM appears more difficult than establishing 2D TFM methods, reconstitution of 3D force fields in a homogeneous 3D hydrogel in fact can be easier than achieving it on a 2D compliant surface, as it is convenient to directly interpolate the hydrogel displacement field at the cell-ECM interface from bead displacements around the cell, thus creating a forward problem for calculating cellular contractile forces at the cell-ECM interface. ${ }^{[223]}$ Achieving the same for 2D hydrogel surfaces in 2D TFM methods requires extrapolation, the accuracy of which can be a concern.

TFM has been successfully applied to study both cell-ECM and cell-cell interactions in different biological questions involving cellular contractile forces (Figure 10a, bottom panel), revealing for example, correlation of local extracellular contractile forces with the orientation, total fluorescence intensity and area of FAs and coordination and interdependency of cellular contractile forces at cell-ECM and cell-cell adhesions. ${ }^{[228]} \mathrm{A}$ recent new trend is to apply TFM to study cell-ECM and cellcell force patterns within a cluster of cell undergoing collective cell migration. Such studies have helped reveal that cellular contractile forces driving collective cell migration can arise predominately many cell rows behind the leading front edge and extend across enormous distances, which is contrary to the conventional theory emphasizing the important role of contractile forces arising from leader cells. ${ }^{242,243]}$

In addition to studying cell-ECM and cell-cell mechanical interactions under static homeostasis, a recent trend applied TFM to investigate the role of cellular forces in cell behaviors under dynamics conditions mimicking physiological stimuli, e.g., shear flow, mechanical stretch and chemokine gradient, by integrating the TFM with miniaturized mechanically actuating system.

For example, in the study by Shiu and colleagues, integrated 2D TFM was used to characterize endothelial cell-ECM mechanical crosstalk under shear flow and revealed significant enhancement of cell migration as well as cellular contractile forces, which were mediated by elevated intracellular RhoA/ ROCK signaling under shear stress. ${ }^{[244]}$ In another study by Hur and colleagues, the hybrid TFM was deployed in a fluidic channel, where either laminar flow or oscillatory flow was generated (Figure 10d). ${ }^{[245]}$ Using this integrated setup, in combination with the assumption of mechanical equilibrium within and in between cells, they evaluated not only the cell-ECM force but also cell-cell (intercellular) and intracellular forces in 3D. Under laminar flow, they observed a significant coordination between intracellular force polarization and cell morphological remodeling. However, the remodeling of both intracellular force and cell shape was insignificant when oscillatory flow was applied. More interestingly, they found that ECs at subconfluence behaved somewhat differently from confluent cells, wherein the latter had larger cell-cell force along the outof-plane direction, suggesting an emergent role for adherence junctions (AJs). Therefore, a confluent monolayer of ECs might be a better model system for future studies of endothelial mechanobiology under shear flow.

Instead of the shear flow, the chemokine gradient in blood vessel regulates the directional migration of another group of cells, e.g., DCs, leukocytes, neutrophils. In order to study how cell-ECM mechanical crosstalk plays a role in chemotaxis, researcher used 2D TFM and micropipetted chemoattractants in earlier studies (Figure 10e). ${ }^{[236,246]}$ This method was effective and as well delivered lots of understanding about modes of cell motility and the role of cell adhesion proteins and motor proteins in regulating the chemotaxis.

In order to understand how cell-ECM interaction behaves under mechanical stretch, Gavara and colleagues adapted 2D TFM onto an elastomeric membrane, which was further integrated into a cell-stretching device (Figure 10f). Using this novel integrated 2D TFM, they observed an inelastic, stretch-dependent cellular force recovery after a transient stretch is applied and released, wherein the recovery scope of cellular force decreased at increasing stretch ratio. ${ }^{[247]}$ At subcellular level, the recovery dynamics of local contractile force was found dependent of not only the stretch ratio but also the baseline traction before the stretch was applied. It reflected a history-dependent, viscoelastic nature of cells remodeling intracellular structures, e.g., actin CSK and FAs, in response to a transient stretch.

As a powerful and convenient method, TFM has contributed significantly to mechanobiology research and will continue to be important in characterizing cell-ECM and cell-cell interactions, critical for understanding the functional roles of mechanical forces involved in mechanoresponsive cell adhesion, migration and differentiation in both 2D and 3D contexts.

Elastomeric Micro/nanoscale Beams as Strain Sensors. An alternative to TFM to visualize the deformation of the surface underneath a contractile cell is to generate micro/nanoscale beam or post structures that can bend or deflect as strain sensors while serving as a cell adhesive surface. The elastomeric PDMS micro/nanopost array reviewed in section 2.1.2 was an excellent example among others for such a purpose. ${ }^{[105,114,248]}$ When adherent cells are cultured on the top surface of a fluorescently labeled PDMS micro/nanopost array (Figure 11a), the cells can pull and bend the post tops, displacements of which 


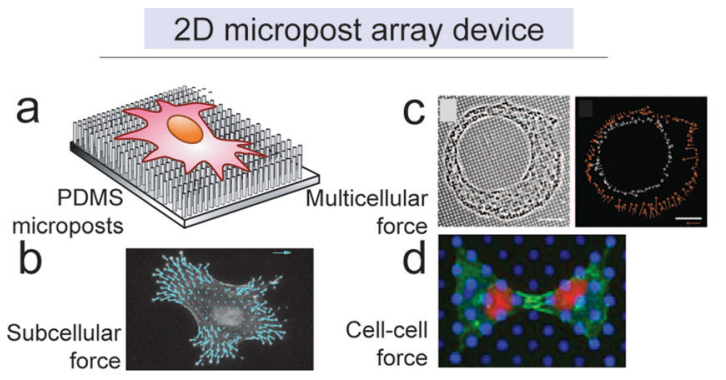

\section{Integrated microposts in fluidic environment}

g

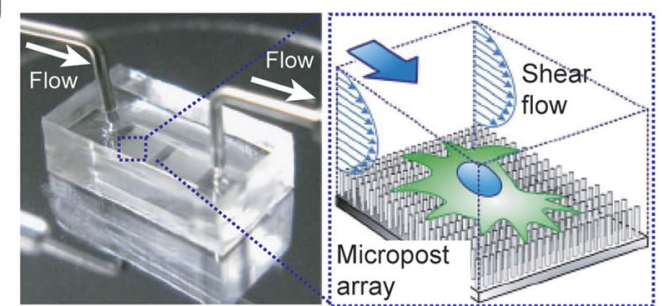

h Chemokine $20 \mathrm{nM} \mathrm{CCL19}$ solution inlets $0 \mathrm{nM} \mathrm{CCL19}$

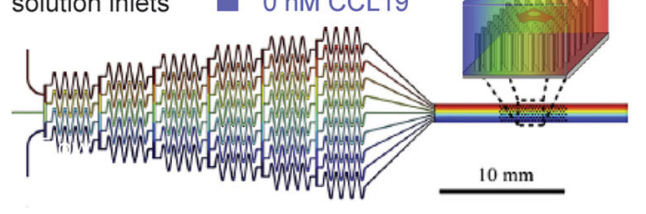

2D microtissue force gauge

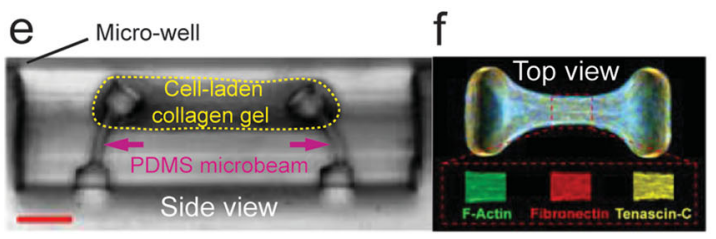

Characterizing microtissue contraction

\section{Integrated microposts in dynamic stretch device}

i
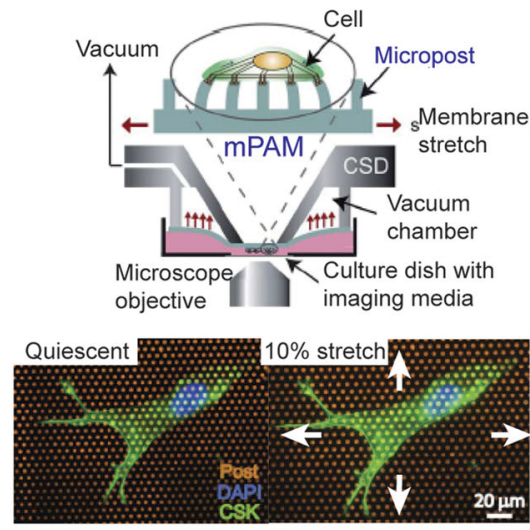

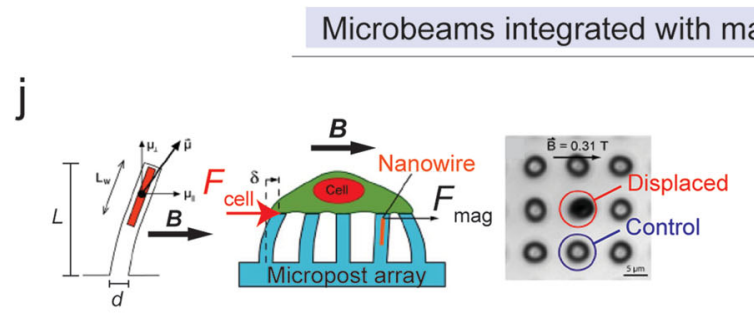

Microbeams integrated with magnetic actuation mechanism

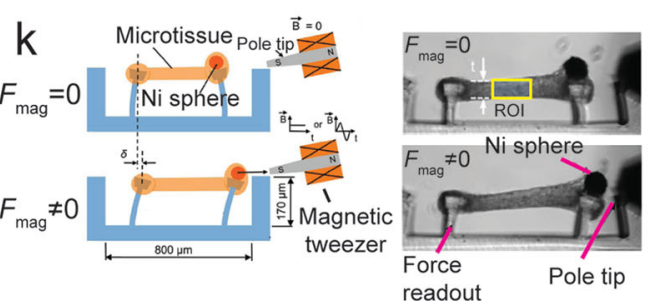

Figure 11. Quantification of cellular contractility by elastomeric microbeam bending. (a) Schematic of the micropost array for monitoring cell-ECM contractile forces. (b) Processed fluorescent image showing subcellular cellular contractile forces within a single cell. ${ }^{[108]}$ Reproduced with permission. ${ }^{108]}$ Copyright 2011, Nature Publishing Group. (c) Phase contrast image (left) and processed traction force map (right) showing cell-ECM forces underneath a micro-patterned multi-cell cluster. ${ }^{[249]}$ Reproduced with permission. ${ }^{[249]}$ Copyright 2007, United States National Academy of Sciences. (d) Fluorescent image showing the application of the micropost array in studying cell-cell tugging force. ${ }^{[227]}$ Reproduced with permission. ${ }^{[227]}$ Copyright 2010 , United States National Academy of Sciences. (e) Cross-section view of the microtissue force gauge ( $\mu$ TUG). ${ }^{[250]}$ (f) Top view of the dog-bone like microtissue formed between two heads of microbeams after the gelation and contraction of cell-laden collagen gel.[250] Reproduced with permission. ${ }^{[250]}$ Copyright 2009, United States National Academy of Sciences. (g) Picture (left) and schematic (right) of a microfluidic channel with integrated micropost array for monitoring the dynamics of cell-ECM contractile forces during endothelial cell morphological remodeling under laminar shear flow. ${ }^{[253]}$ Reproduced with permission. ${ }^{[253]}$ Copyright 2012, Royal Society of Chemistry. (h) Schematic of a microfluidic gradient generator with integrated micropost array for studying the dynamics of cell-ECM mechanical crosstalk during dendritic cells chemotaxis. ${ }^{[254]}$ Reprodued with permission. ${ }^{[254]}$ Copyright 2011 , Cell Press. (i) Schematic (upper) and fluorescent images (bottom) of a PDMS micropost array integrated with a cell-stretching device for studying the contractility of vascular smooth muscle cells under equibiaxial stretch. ${ }^{[255]}$ Reproduced with permission. ${ }^{[255]}$ Copyright 2012, Royal Society of Chemistry. (j) Magnetic micropost array. ${ }^{[262]}$ (left) Schematic of magnetic actuation using Co nanowire embedded in a PDMS micropost. (middle) Schematic of monitoring cell-ECM contractile forces during the magnetic actuation. (right) Phase contrast image of the bending of magnetic micropost under magnetic field. Reproduced with permission. ${ }^{[262]}$ Copyright 2007, United States National Academy of Science (k) Magnetic microtissue force gauge. ${ }^{[264]}$ (left panel) Schematic of magnetic actuation using Ni sphere incorporated within a microtissue. Either static or cyclic actuation could be achieved using the magnetic tweezers. (right panel) Cross-section view of the microtissue before and after magnetic actuation. The region of interest (ROI) was selected as the central part of the microtissue, and subsequent biomechanics analysis could be performed to extract the mechanical properties of the microtissue. Reproduced with permission. ${ }^{[264]}$ Copyright 2012, Wiley-VCH (Germany). 
can be recorded using fluorescence microscopy and further converted to tangential cellular contractile forces. As dictated by the beam theory, cellular contractile forces applied to each post is evaluated from bending displacements of post tips, in combination with the post spring constant. ${ }^{[05]}$ The discrete top surface presentation of the PDMS micro/nanopost array can have certain effects on benchmark cell behaviors (e.g., cell spreading and proliferation); however, such effects can be minimized by reducing the PDMS post center-to-center distance. So far, comparable cell behaviors have been observed for fibroblasts, endothelial cells, smooth muscle cells, MSCs, and ESCs, among others, when cultured on the PDMS micro/nanopost array and continuous culture surfaces. ${ }^{[105,108,110,139,227]}$ The PDMS micro/nanopost array has been successfully applied in many studies investigating cell-ECM force interactions at both subcellular and cellular levels (Figure 11b,c). ${ }^{[107,108,249]}$ Similar to TFM, the PDMS micro/nanopost array was also used to study cell-cell tugging force and its role in E-cadherin-mediated AJ formation and remodeling by assuming a mechanical equilibrium between cell-ECM and cell-cell forces (Figure 11d).[227]

The PDMS micro/nanopost array has been primarily used for characterizing cell-ECM mechanical interactions on 2D surfaces at subcellular and cellular levels. To study cellular forces within 3D ECM at a tissue level, Legant and colleagues recently developed a multilayer photolithography process to fabricate bone-shaped PDMS microbeams embedded in recessed microscale wells as microfabricated tissue force gauges (" $\mu$ TUGs") (Figure 11e). ${ }^{[250-252]}$ Such $\mu$ TUGs were used to simultaneously constrain the remodeling of a collagen gel embedded with contractile cells and to report the microtissue contraction generated during this process. After the microscale well was immersed in a suspension of contractile cells and unpolymerized type I collagen and centrifuged to drive cells into the recessed well, a cell-laden microtissue formed spontaneously upon gelation, during which collagen fibers intertwined and contracted against the microbeams and evolved eventually into a dog-bone-like microtissue pulling against the microbeam heads (Figure 11f). Although not able to resolve forces at the cellular or subcellular level, an elegant feature of the $\mu$ TUG lies in its ability of characterizing dynamic contractile behavior of microscale tissues encapsulated with mammalian cells while observing matrix remodeling at the cellular length scale. The TUG can also allow independent modulations of mechanical stiffness of the PDMS microbeams and ECM matrix, which was utilized by Legant and colleagues to study the relationships between cellular and matrix mechanics, cellular forces, and cytoskeletal and ECM protein expressions within a 3D microenvironment. ${ }^{[250-252]}$

Like TFM, the microbeam-based strain sensors have also been adapted recently to study cell-ECM interactions under physiology-mimicking dynamical conditions, through integration with miniaturized actuating systems. For instance, Lam and colleagues recently integrated the PDMS micropost array into a microfluidic channel, in order to measure the dynamics of cell-ECM contractile forces in response to laminar shear flow (Figure 11g).[253] Fabricated by soft lithography, the micropost array was functionalized with ECM proteins and then sealed into a microfluidic channel via plasma-assisted bonding. Laminar flow was applied when endothelial cells (ECs) were at subconfluence on microposts. In their study, the cell-ECM contractile force showed a dynamic profile closely coordinated with cell morphological remodeling induced by the shear stress, making both of them gradually lined up with the flow direction.

In order to study cell-ECM mechanical crosstalk during chemotaxis, recently, Ricart and colleagues integrated a microfluidic gradient-generator with the micropost array force sensor (Figure 11h). ${ }^{[254]}$ In their study, they found the contractile force of DCs was correlated with the migration speed. However, the total cell-ECM force was found rather self-sustained while showing little response to change in chemokine gradient. It suggested that the regulation of cell contractility might be separated from that of the chemokine-sensitive migration direction. Their study also revealed the subcellular pattern of cell-ECM force was closely correlated with the orientation of migration, wherein the maximum principle force was always seen in the front of cell centroid. These findings demonstrated that an integrated microenvironment with well-defined soluble factor gradient, matrix mechanics as well as functional forcesensing ability could be very useful for future study of the mechanotransduction and mechanobiology of blood-residing immuno-responsive cells.

In addition, Mann and colleagues integrated the micropost array on a memebrane (mPAM), and studied the dynamic tensional homeostasis of VSMCs under step-like stretch (Figure 11i). ${ }^{[255]}$ An interesting biphasic response of VSMC contractility was observed. Moreover, using subcellular information obtained with this method, Lam and colleagues reconstituted intracellular deformation and force response field under stretch, and evaluated cell stiffness at subcellular resolution. ${ }^{[256]}$

Besides these miniaturized mechanically actuating systems, the microbeam strain sensors have also been integrated with magnetic actuation mechanisms, in order to study how cellular force and other properties respond to local mechanical perturbations applied directly through surrounding ECM, rather than via foreign materials such as AFM tip or antibodyconjugated microbeads. ${ }^{[257-261]}$ Recently, Sniadecki and colleagues achieved this goal by integrating magnetic nanowire actuation scheme to a previously developed micropost array that was used to modulate substrate rigidity as well as to monitor cell-ECM forces (Figure 11j). ${ }^{[262]}$ Magnetic Co nanowires, fabricated via electrochemical deposition, were integrated into PDMS microposts during soft lithographical fabrication. In the presence of external magnetic field, the nanowire embedded at the tip of a micropost was subject to a torque and bended the post, and thus imposed a local external force only to the FA anchored on the same post. In their study, the force-dependence of FA protein recruitment was found a local property of this mechano-sensing machinery, elaborating a previous discovery that FAs are general force-sensing structures. ${ }^{[263]}$ In addition, the subcellular cell-ECM contractile forces in response to the local mechanical stimulus was monitored in the meantime and revealed an interesting "nonlocal" pattern, wherein the magnetic field-driven bending of a micropost instantly and effectively reduced the contractile forces on neighboring posts. Immunofluorescence suggested that the local external force "plucked" the stress fiber bridging over several microposts nearby and spread the stimulation out. 
In another example, Zhao and colleagues applied the idea of magnetic micropost array to the recently developed microtissue force gauge (Figure 11k). ${ }^{[264]}$ In their device, they put a microscale Ni sphere on top of either microbeam. When the microtissue was assembled in situ during the gelation and contraction of collagen gel laden with 3T3 fibroblasts, the Ni sphere was incorporated as well. Under external magnetic field, the Ni sphere was pulled toward the magnetic pole, either statically or cyclically, and the contractile response of the microtissue was measured by the bending of the other microbeam. This integrated magnetic force gauge presented an analogy of traditional mechanical tension test, but at a much smaller scale and designed specifically for reconstituted microtissues. It enabled a straightforward characterization of microtissue biomechanics. By quantifying the deformation field within the microtissue using imaging analysis, Zhao and colleagues also studied the biomechanics of microtissue. In addition, in the presence of cyclic external stretch, they observed a mechanical conditioning of the microtissue stiffness, which was reversible once the stretch was removed. Although it lost the ability to directly monitor forces at cell-ECM and cell-cell interfaces, this integrated microscale bio-actuator and force sensor enabled measurement of the overall contractile phenotype of the microtissue in response to either static or cyclic external stretch, as well as characterization of microtissue mechanics. This integrated microtissue force gauge provided a potential method that could quantify the contraction of cardiac muscle or artery smooth muscle constructs under physiological-like stretch, and help understand how the functions of these muscle tissues are conditioned by force, which has great implication in many cardiovascular diseases such as hypertension. ${ }^{[52]}$ In addition, it also enabled potential modulation of gel stiffness (by crosslinking or degradation), cell-gel interactions (by pharmacological treatment) and the microbeam rigidity (by geometrical modulation), mimicking the mechanical microenvironment that muscle tissues might experience in physiological or pathological conditions, and therefore help explore how different microenvironmental cues, besides the contraction itself, regulate muscle tissue functions and inflammation in a combinatorial manner.

Molecular Force Sensors Using Single Molecule Fluorescence Force Spectroscopy. Although powerful, TFM and elastomeric micro/nanoscale post and beam structures discussed above can neither achieve cellular force measurements down to a molecular level nor provide direct information regarding specific receptor-ligand interactions mediating cell-ECM force interactions. Further, elastomeric micro/nanoscale post and beam structures, owing to their inherent fabrication methods, cannot be generated on curved surfaces, which are in some cases necessary for mimicking adherent cells in the in vivo microenvironment. Over the last few years, different emerging molecular force sensors using fluorescence proteins have been successfully developed based on the principle of force-driven molecular conformation changes to achieve real-time force measurements in living cells. These molecular force sensors can be coupled with FRET (fluorescence resonance energy transfer) or distance-dependent fluorescence quenching to allow using live-cell fluorescence imaging to probe extracellular and intracellular forces in the range of $1-50 \mathrm{pN}$, sufficient to drive conformational changes in macromolecules and molecular assemblies.
The mechanism of fluorescence resonance energy transfer (FRET) involves a donor fluorophore in an excited electronic state, which may transfer its excitation energy to a nearby acceptor fluorophore in a non-radiative fashion through longrange dipole-dipole interactions. The efficiency of FRET is dependent on the inverse sixth power of the intermolecular separation between donor and acceptor fluorophores. Thus, FRET is a very useful technique for investigating a variety of biological phenomena that produce changes in molecular proximity in the range of $1-10 \mathrm{~nm}$ in living cells. ${ }^{[265]}$

Recently, Grashoff and colleagues developed a genetically encoded FRET-based vinculin force sensor with single piconewton sensitivity for use in living cells to study force transmission across vinculin and its role in FA dynamics. ${ }^{[266]}$ This vinculin force sensor, the first of its kind for use in molecular force measurements in living cells, was developed by inserting between the head and tail domains of vinculin a pair of FRET donor and acceptor fluorophores that were linked by an elastic amino-acid domain derived from the spider silk protein flagelliform to serve as an entropic nanospring suitable for measuring piconewton forces (Figure 12a). Using the vinculin force sensor, Grashoff and colleagues demonstrated for the first time in living cells that force across vinculin in stable FAs was about $2.5 \mathrm{pN}$ and that vinculin recruitment to FAs and force transmission across vinculin were controlled independently, with highest force transmitted across vinculin associated with adhesion assembly and enlargement while low forces across vinculin in disassembling or sliding FAs at the trailing edge of migrating cells (Figure 12b).

Genetically encoded protein force sensors are particularly powerful for measuring intracellular biophysical forces in vivo. It should be noted, however, that the force sensing module inserted into different force-bearing proteins will need custom design and careful characterization to ensure the wild-type function of the host proteins can be regained upon splitting and insertion of the force sensor into the host protein. ${ }^{[118,267,268]}$ Moreover, the application of intracellular molecular force sensors based on FRET were restricted in some situations by the short working distance $(<10 \mathrm{~nm})$ dictated by the physical principle of FRET. For example, talin, an important mechanosensitive and -transductive protein within the FAs, has a native length about $50 \mathrm{~nm}$ and could be extended up to $800 \mathrm{~nm}$ (in theory) under actomyosin contractile forces, which largely exceeds the working range of FRET. In order to measure the tensile deformation within single talin 1 proteins, and thus to reveal the force-dependent talin 1 conformational dynamics during mechanotransduction, Margadant and colleagues developed a dually tagged Talin, EGFP-N-Talin1-C-mCherry, whose head-tail distance could be directly measured using dual channel fluorescence microscopy and advanced image processing techniques. ${ }^{[269]}$ Using the dually tagged talin1 molecular strain sensor, Margadant and colleagues found individual talin underwent repeated stretch-relaxation with a period about 10-15 s, and the spatial feature of such fast molecular conformational dynamics was conjugated with local rearward flow of actin and could be suppressed by the binding of vinculin to talin through cryptic sites unveiled during the stretch on talin. Their results suggested a highly dynamic mechanotransduction event in situ through force-dependent conformational change of talin1 and its structural integration with vinculin. 

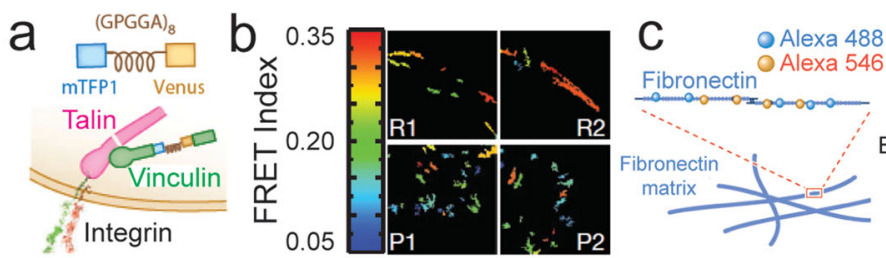

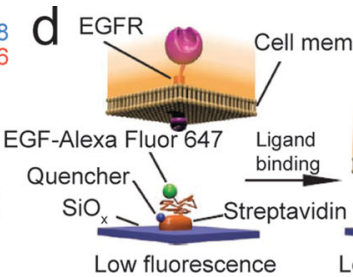

Low fluorescence

\section{e}

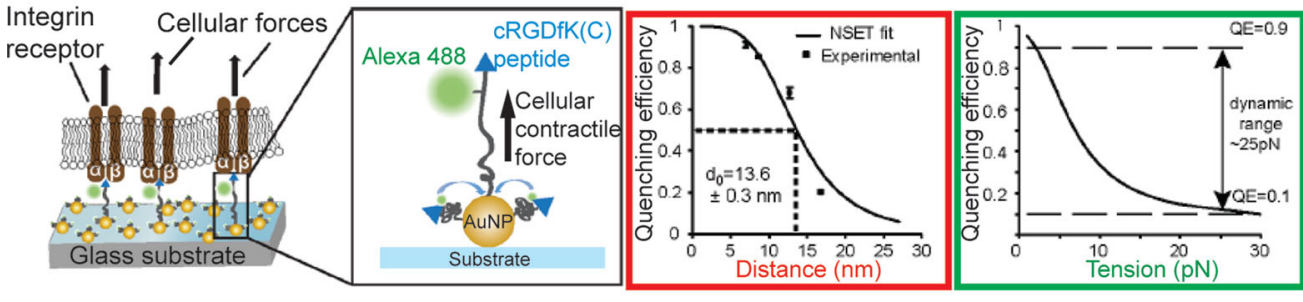

Deformation and Tension-dependent fluorescence quenching

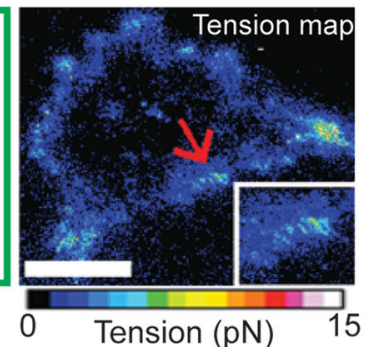

f

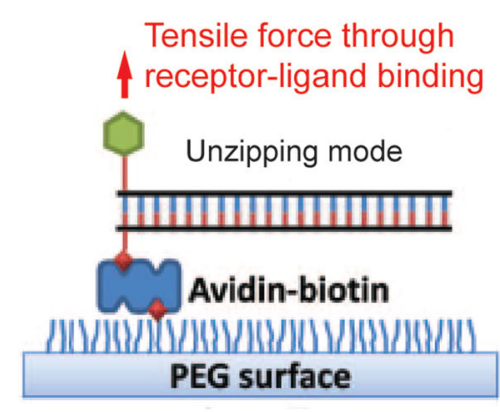

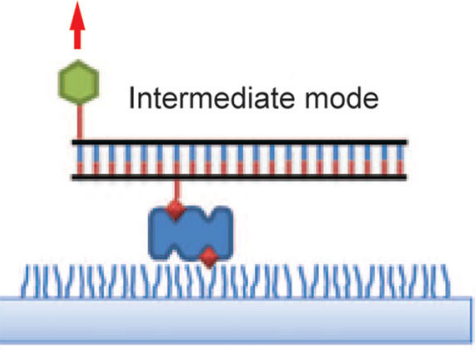

Tension tolerance

High

Figure 12. Nanoengineered fluorescent single-molecule force/strain sensors. (a) Schematic of the FRET vinculin force sensor. ${ }^{[18]}$ The head and tail of vinculins were grafted with FRET donor and acceptor, respectively. Reproduced with permission. ${ }^{[18]}$ Copyright 2013, Annual Reviews. (b) Images showing FRET index of vinculin sensors in the protrusions (P) and rear (R) of a cell. [266] Higher FRET index (efficiency) means shorter distance between vinculin head and tail and thus correlates with smaller tensional force within vinculins. Reproduced with permission. ${ }^{266]}$ Copyright 2010, Nature Publishing Group. (c) Schematic of a FRET fibronectin strain sensor. ${ }^{[18]}$ Multiple donors and acceptors were incorporated into each individual molecule, in order to expand the dynamic range of FRET. Reproduced with permission. ${ }^{[18]}$ Copyright 2013, Annual Reviews. (d) Schematic of an extracellular molecular force sensor based on FRET mechanism. The fluorophore was conjugated to the ligand that was immobilized on the substrate in proximity to a quencher. When there was no tension through the binding between the ligand and the receptor on cell surface, FRET took place and no fluorescence came out from the fluorophore. While tension was applied via the ligand-receptor binding, the fluorophore was pulled away from the quencher and its fluorescence intensity increased. ${ }^{[273]}$ Reproduced with permission. ${ }^{[273]}$ Copyright 2012, Nature Publishing Group. (e) Design and performance of a mechano-sensing nanoparticle. ${ }^{[275]}$ (left two) Schematic of the mechano-sensing gold nanoparticle (AuNP). Tensional force transmitted via integrin pulled the Alexa 488 fluorophore away from the AuNP and released it from fluorescence quenching. (middle two) Distance-dependent quenching efficiency of the fluorescence and the mapping of quenching efficiency to tensional force on the RGD-functionalized PEG chain. (right) Subcellular tension map underneath an adherent cell. Reproduced with permission. ${ }^{[275]}$ Copyright 2013, American Chemical Society. (f) Schematic of the design of single molecular force sensors that modulate the tension tolerance (rupture force) by adjusting the positions of ligand and substrate anchorage, respectively, and thus shifting the fracture mode of the molecules under cellular tensional force. ${ }^{[276]}$ Adapted with permission. ${ }^{[276]}$ Copyright 2013 , American Association for the Advancement of Science.

In addition to intracellular molecular force sensors, FRET has also been applied to develop extracellular molecular force sensors. In a work reported by the Vogel group, an extracellular FRET-based molecular force sensor based on fibronectin (FN) was developed. ${ }^{[270,271]}$ Since the working range of FRET is only between 1-10 nm, Banyex and colleagues integrated multiple donor and acceptor fluorophores into individual FN molecules, where FRET interactions between multiple donors and acceptors could broaden the effective working distance of FRET and render it capable to report large deformation of FN molecules (Figure 12c). ${ }^{[270]}$ Using FRET-based FN sensor, FN extension, relaxation and remodeling were studied in a force-dependent manner. ${ }^{[251,272]}$

More recently, Stabley and colleagues, for another example, developed a FRET-based extracellular molecular force sensor for spatially and temporally mapping forces exerted by cell-surface receptors (Figure 12d). ${ }^{[273,274]}$ This molecular sensor consisted of a flexible linker that was covalently conjugated to a biological 
ligand at one terminus and anchored onto a surface (via a biotin-streptavidin interaction) such that mechanical forces could not result in sensor translocation. The linker used by Stabley and colleagues was comprised of a PEG polymer given its well-characterized and reversible force-extension curves as well as its biocompatibility and minimal nonspecific interactions with other biomolecules. The ligand and the surface were functionalized with fluorophore and quencher molecules, respectively. Thus, when cellular forces exerted on the ligand extended the linker from its relaxed conformation and removed the fluorophore from proximity to the quencher, fluorescence intensity of the fluorophore tethered to the ligand would increase and provide a signal to map mechanical force transduced through specific receptor targets. Notably, the approach developed by Stabley and colleagues is noninvasive and can be used to map forces for cell surface receptors in living cells. Their method only requires the use of a conventional fluorescence microscope and precludes the necessity of genetic engineering of target receptors. Based on a similar configuration, in a follow-up work from the same group, Liu and colleagues further developed a gold nanoparticle (AuNP)-based extracellular molecular sensor utilizing distance-dependent fluorescence quenching between maleimide-Alexa488 fluorescence dye and AuNP (Figure 12e). ${ }^{[275]}$ The ligand and the surface in this work were functionalized with Alexa488 fluorescence dye and AuNP, respectively. Distance-dependent fluorescence quenching by AuNP is dependent on the inverse fourth power of the intermolecular separation. Thus, AuNP-based extracellular molecular sensors, in principle, can extend the detection range for molecular deformation to $\sim 20 \mathrm{~nm}$, which outperforms the FRET detection mechanism. ${ }^{\text {[275] }}$

By applying these extracellular/intracellular force/strain sensors, mounting evidence has been found in recent decade revealing a mechanosensing nature of living cells that relies much on the cellular contractile force, which has been shown required for many mechano-responsive cell behaviors. Although it has long been postulated that a certain amount of force through cell adhesion receptors (e.g., integrins) is required to activate integrin, form mature FAs and dictate cellular sensitivity to ECM properties such as the rigidity, there was no clear evidence until recently that quantitatively demonstrated the minimal single molecular receptor-ligand force that is necessary for living cells to deploy adhesion-dependent machineries. In this very recent study, Wang and Ha developed a set of single molecular force sensors that restrict the rupture force (or tension tolerance) of the sensors under external tension (Figure 12f). ${ }^{276]}$ These sensors was designed based on a short DNA duplex helix, of which one chain was connected to the substrate through a single-point anchorage via avidin-biotin binding, while the other chain was point-functionalized with RGD ligand. By changing the positions of the anchorage site and the RGD-functionalized site, the DNA duplex helix undergoes different modes of fracture when subject to contractile force through the integrin-RGD binding and therefore works as a single molecular force sensor (tension gauge tether, or TGT) that could achieve a range of rupture forces from $12 \mathrm{pN}$ to $56 \mathrm{pN}$. Using this novel set of molecular force sensors, they provided quantitative evidence that a minimal single molecular force about $40 \mathrm{pN}$ was required for cells to activate integrin and trigger normal cell adhesion and spreading, proving that a threshold force transmitted through integrin is critical for cellular behaviors. In addition, they found that another threshold force less than $12 \mathrm{pN}$ through single Notch ligand DDL1 was required for Notch activation in well-spread cells.

\subsection{Microengineered 3D Tissue Scaffolds}

The in vivo tissue microenvironment is $3 \mathrm{D}$ in nature. To recapitulate the 3D tissue physiology in vitro for studying disease mechanisms, tissue engineering and drug development, numerous microengineering methods to generate functional 3D tissue scaffolds using human cells combined with scaffolds or devices that facilitate cell growth, organization and differentiation have been developed over the last two decades. These 3D tissue scaffolds with well controlled scaffold architectures, biochemical functionalities and mechanical properties have been implemented to meet the increasing demand for in vitro models that capture more of the relevant in vivo complexity than traditional 2D cultures can achieve. It has been well documented that many external cues in 3D tissue microenvironment including those that arise from various ECM components, mechanical stimulation and soluble signals can affect cellular behaviors in ways different from those 2D substrates do. ${ }^{[3,36,277]}$ Specifically, between traditional 2D cultures and 3D ECM environment, there are differences in the spatial presentation of topographical and biochemical cues, ${ }^{[35]}$ the available free space for cell spreading and migration, ${ }^{[121,131]}$ the amount of ECM available for cell adhesion and contraction, ${ }^{[278]}$ and the molecular composition, morphology and even signaling of cellECM adhesion structures. ${ }^{[277]}$ Furthermore, culturing human cells in microengineered 3D tissue scaffolds has become an indispensable method for tissue engineering and establishing in vitro tissue/organ/disease models for studying disease mechanisms and drug development. ${ }^{[33]}$ Over the last two decades, rapid developments in different micro-engineering and -fabrication techniques have provided various promising 3D scaffold platforms for cell mechanobiology, tissue engineering and drug screening applications.

Microfluidic 3D Scaffolds. Hydrogels, owing to their biocompatibility and 3D porous nature to encapsulate cells as well as to permit diffusion of soluble factors, have been the most popular functional biomaterials used for microengineering 3D scaffolds. Soft lithography, with its versatile microfabrication strategies, was the first method used to engineer 3D scaffolds with hydrogels. ${ }^{[279]}$ Using soft lithography, microfluidic channels and cavities have been successfully fabricated within 3D hydrogels as in vitro platforms for studying angiogenesis as well as engineering microscale vascular structures. ${ }^{[48,280-288]}$ Two strategies are commonly employed for generating microfluidic 3D scaffolds using soft lithography (Figure 13). ${ }^{[48]}$ The first one generates patterned hydrogel structures via replica molding from negative PDMS molds before the hydrogel structure is assembled with other hydrogel substrates using thermal and mechanical methods or chemical perturbants to form monolithic hydrogel structures (Figure 13a-c). ${ }^{[289-291]}$ In the second method, patterned Matrigel structures pre-seeded with cells generated from replica molding are used as a sacrificial 

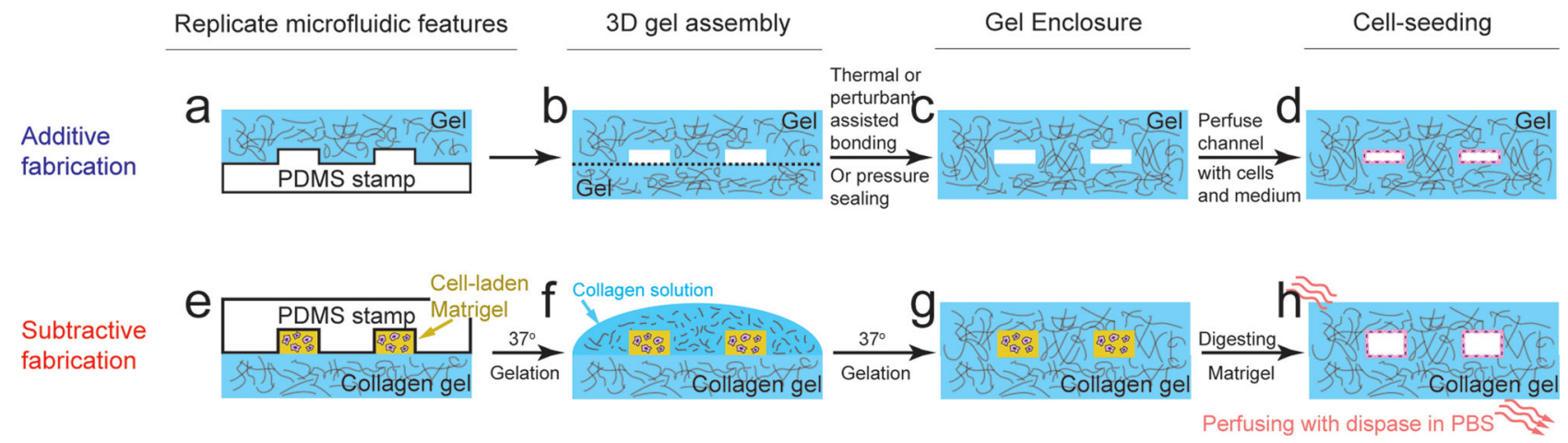

Figure 13. Fabrication of microfluidic 3D scaffolds with hydrogels. Schematic of the additive (a-d) and subtractive (e-h) fabrications of microfluidic 3D scaffolds with hydrogel biomaterials, ${ }^{[48,279,284]}$ which provided appropriate platforms for studying epithelial/endothelial cell-stromal cell interactions as well as vascular physiology and morphogenesis. In common, both fabrication methods take four steps: (1) using hydrogel precursors to replicate the microfluidic features from a PDMS mold using soft lithography; (2) after gelation, the hydrogel replicate is assembled with other hydrogel substrates, which, if repeated, might eventually achieve a multi-layered 3D hydrogel scaffold; (3) the assembled hydrogel scaffold should be sealed (thermally, chemically or mechanically) before perfusing culture medium and cells; (4) perfusing the microfluidic channels with culture medium and cells. See text for more descriptions.

material to be enclosed in a bulk collagen gel (Figure 13e-g). After digesting the Matrigel by perfusing the collagen gel with dispase, microchannels are created in the collagen gel and pre-seeded cells, if any, in the Matrigel are released into the microchannels (Figure 13h). ${ }^{[280]}$ Instead of using soft lithography and cell-laden Matrigel as the sacrificial material, a single microneedle embedded in hydrogels was also used to create individual microchannels with simple geometries in hydrogels. ${ }^{[284,287]}$

As a common protocol, by flooding microfluidic channels enclosed within hydrogels with media containing cells, endothelial cells (ECs) can be seeded onto the lumen of these microfluidic channels to form a EC monolayer mimicking endothelium in vivo (Figure 13d). To mimic in vivo vascular microenvironment, perivascular cells such as pericytes (PCs) or vascular smooth muscle cells (VSMCs) can also be encapsulated into the bulk hydrogel surrounding the microchannels. ${ }^{[285,292]}$ In a recent work by Zheng and colleagues, such microfluidic 3D scaffolds in conjunction with co-cultures of different cell types have allowed for detailed studies of endothelial permeability and angiogenic sprouting under a combinatorial regulation by soluble factors and EC-PC interactions. ${ }^{[285]} \mathrm{In}$ addition to mechanistic studies of vascular physiology, microfluidic 3D scaffolds seeded with ECs have also served as disease models for studying thrombosis by introducing whole blood to the EC lumen while simultaneously monitoring EC inflammatory responses such as their interactions with platelets and leukocytes as well as formations of von Willebrand factor (VWF) fibers on ECs. ${ }^{[285]}$

In another study, Nguyen and colleagues developed a microfluidic 3D hydrogel scaffold where ECs were seeded in one of two closely positioned microfluidic channels with the other filled with media containing soluble angiogenic factors. ${ }^{[286]}$ Under stimulation from angiogenic factors, ECs were observed to sprout and migrate away from the EC lumen toward the other microchannel, and many in vivo hallmarks of angiogenesis were successfully reconstituted using this microfluidic 3D hydrogel scaffold. This in vitro angiogenesis model was demonstrated to be specifically useful for studying the morphogenic process step by step during angiogenesis, as well as for screening how different angiogenic factors and drug inhibitors could function independently or in a combinatory fashion to promote or antagonize angiogenesis.

Besides 3D hydrogel scaffolds generated using microfluidics and soft lithography, some cell-laden microstructures enclosed within a functional hydrogel (as illustrated in Figure 13g) were also shown as promising for tissue engineering and drug development. Recently, Baranski and colleagues used a microtissue molding approach to demonstrate that the geometrical feature of microengineered endothelial cords embedded in fibrin gels could affect their integration with host circulation and tissue vascularization. ${ }^{[293]}$ In their in vivo study, improved survival and function of hepatocyte constructs were observed only for those microscale tissues containing highly aligned endothelial cords, which promoted host vascular response, vessel maturation and generation of new capillaries. Together, these recent studies have revealed the exciting promise of microengineered 3D hydrogel scaffolds for studying multicellular interactions and architecture in tissue integration and function, disease mechanisms, and drug development. ${ }^{[48]}$

Non-Traditional Methods for 3D Microfluidic Vascular Networks. Soft lithography and microfluidic techniques have been commonly used to form planar vascular networks in hydrogels, which may then be stacked to produce 3D patterned microchannel networks. ${ }^{[279]}$ However, to form synthetic microvascular networks composed of complex, high-density, hierarchical 3D architectures throughout a thick slab of hydrogel, a milestone necessary for the production of thick pre-vascularized soft tissue constructs or other large scale biomimetic microfluidic materials, soft lithography and microfluidic techniques may not be adequate, as this will require a large number of micropatterned thin hydrogel layers to be aligned and stacked. The impracticality of this process largely limits the use of multilayer stacking to produce complex 3D microfluidic vascular networks within hydrogels. Recently, several non-traditional approaches for generating 3D microfluidic vascular networks have been developed using sacrificial microfiber networks, direct ink writing (DIW), and electric discharge. These novel methods 
have enabled rapid productions of novel functional biomaterials with complex hierarchical 3D microfluidic vascular networks for tissue engineering and regenerative medicine.

Two representative recent studies have both chosen to use sacrificial microfiber networks for rapid casting of vascular networks for microengineered perfusable 3D tissue constructs. In the work by Bellan and colleagues, they fabricated a sacrificial 3D microfiber network using melt-spun shellac, a material that has pH-dependent solubility and thus could be subsequently removed using ammonia bath after the network was embedded within cross-linked gelatin. ${ }^{[24]}$ In addition to the rapid, largescale fabrication, the $3 \mathrm{D}$ connectivity and the dimensions of the microchannels in such a construct were directly modulated by the process of melt-spinning. More recently, Miller and colleagues custom modified a $3 \mathrm{D}$ printer to print rigid $3 \mathrm{D}$ filament networks of carbohydrate glass, and used them as a cytocompatible sacrificial template in engineered tissues containing living cells to generate cylindrical networks that could be lined with ECs and perfused with blood under high-pressure pulsatile flow (Figure 14a). ${ }^{[295]}$ In their method, vascular network pattern was prescribed by directly printing a 3D carbohydrate glass lattice, which was then encapsulated within bulk monolithic cellularized tissue constructs before dissolved with culture media. Vascular ECs were then seeded into the microfluidic network to form endothelial lumen before the vascular network was perfused with blood. Endothelial sprouts were observed to form spontaneously within the patterned 3D microfluidic vascular networks, suggesting efficient functional interactions between ECs and perivascular cells in the tissue (Figure 14b). Since this simple vascular casting approach could allow independent control of network geometry, endothelialization and extravascular tissue, it is compatible with a wide variety of cell types, synthetic and natural ECMs, and crosslinking strategies. To demonstrate practical tissue engineering applications of their method, Miller and colleagues further demonstrated that the perfused vascular channels sustained the metabolic function of primary rat hepatocytes in engineered tissue constructs that otherwise exhibited suppressed function in their core.

The direct ink writing (DIW) was another new method developed recently to fabricate 3D microvascular network in hydrogels (Figure 14c). ${ }^{[296,297]}$ In this method, an F-127 pluronicbased fugitive ink was used to deposit the vascular network directly within a hydrogel precursor solution, which was then liquefied and removed after gelation of the hydrogel, creating a perfusable 3D vascular network (Figure 14d). a Rapid casting

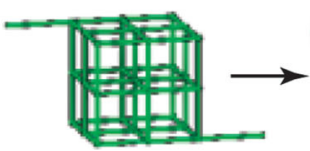

Print 3D carbohydrate glass lattice

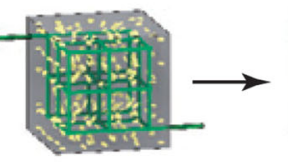

Encapsulating live cells in ECM

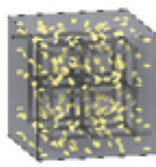

Dissolving lattice with cell medium

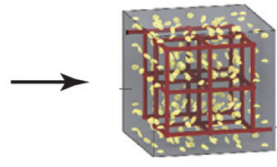

3D ECM with perfusable vascular network b

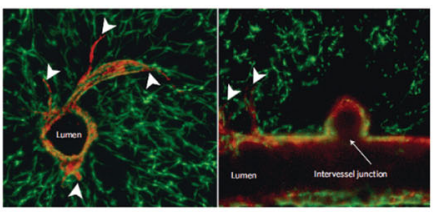

Neovascularization

\section{Direct ink writing $\mathrm{d}$}

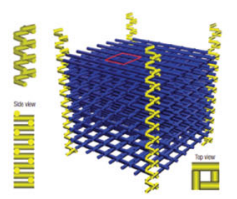

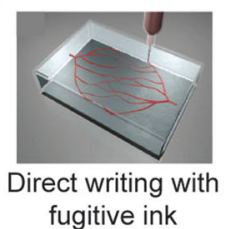

fugitive ink

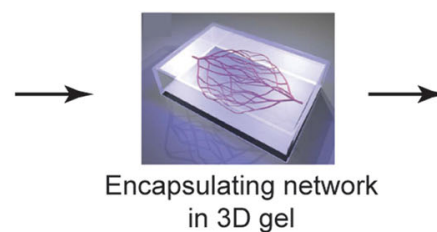

in $3 D$ gel

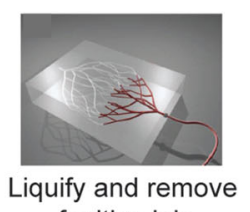

fugitive ink

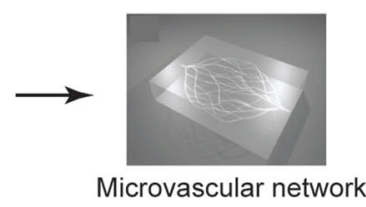

e Electrostatic discharge
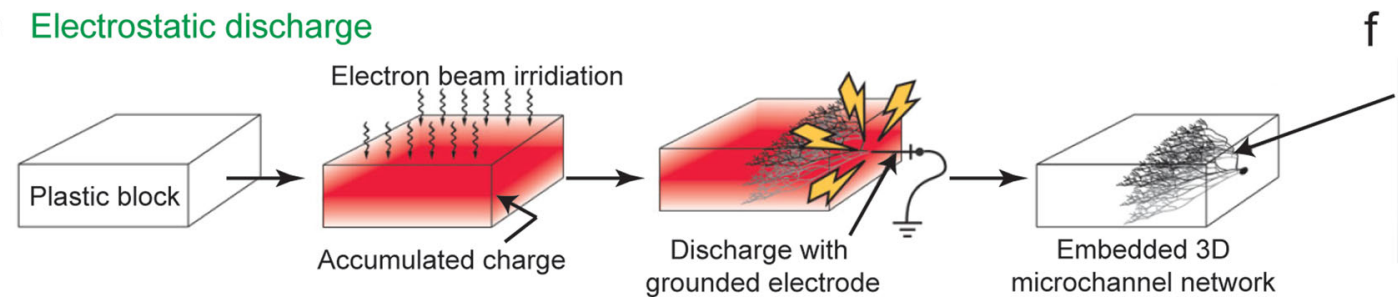

Figure 14. Non-traditional methods for fabricating 3D microfluidic vascular network. (a) Schematic of the process of rapid casting for fabricating 3D perfusable vascular network. ${ }^{[295]}$ (b) Confocal fluorescent micrographs showing neovascularization stemming from pre-patterned vascular network (arrow heads). ${ }^{[295]}$ Reproduced with permission. ${ }^{[295]}$ Copyright 2012, Nature Publishing Group. (c) Schematic of an epoxy-embedded 3D structure fabricated by direct ink writing (DIW).[296] Reproduced with permission. ${ }^{[296]}$ Copyright 2003, Nature Publishing Group. (d) Schematic of the fabrication of a biomimetic microvascular network using DIW in a photo-curable hydrogel reservoir. ${ }^{[297]}$ Reproduced with permission. ${ }^{[297]}$ Copyright 2011 , Wiley$\mathrm{VCH}$ (Germany). (e) Schematic of a rapid fabrication process of 3D hierarchical vascular network via electrostatic discharge in plastic-like materials (e.g., PMMA and acrylic). ${ }^{[298]}$ ( $f$ ) Photograph of a hierarchical vascular network fabricated within an acrylic block. ${ }^{[298]}$ Reproduced with permission. ${ }^{[298]}$ Copyright 2009, Wiley-VCH (Germany). See text for detailed descriptions of the fabrication methods. 
Although both methods we discussed above could be potentially applied to generate microengineered vascular network with a hierarchical geometry mimicking the microvasculature in vivo, the direct printing/writing nature of both methods posed the fabrication time a great limiting factor for such an application. Inspired by the natural fractal tree-like electrostatic discharge path, as seen in the lightening from the sky, Huang and colleagues exploited the hierarchy of electric discharge phenomenon to rapidly create 3D vascular network within plastic-like materials such as PMMA and PLA (poly(lactic acid)) (Figure 14e,f). ${ }^{[298]}$ Upon electron beam irradiation, a large amount of charge was accumulated within the material such that the energy released during the discharge was high enough to vaporize and fracture the surrounding materials along the discharge path. The subsequent electrostatic discharge was initiated either exogenously using a sharp tip of a grounded electrode or spontaneously by a nucleation defect introduced into the material before the irradiation. The rapid and intense release of electrostatic energy instantaneously created a hierarchically branched microchannel network throughout the material, faithfully following the fractal nature of the discharge phenomenon. By repeating the irradiation and discharge processes, Huang and colleagues also created microvascular network of multiple sources/sinks for integration with microfluidic modules.

Layer-by-Layer Cell Patterning for 3D Tissue Scaffolds. Animal tissues and organs generally have composite structures with different types of cells adjacent to one another in a layered fashion within the ECM. Such layered structures of animal tissues and organs in conjunction with in situ cell-cell interactions have long been considered as key regulators of their physiological functions. ${ }^{[33]}$ To probe the role of multicellular organization in $3 \mathrm{D}$ microenvironments in regulating physiological cellular behaviors, it becomes imperative to develop microscale tissue engineering strategies for rapid formation of reproducible, high-resolution 3D multicellular structures. Layer-by-layer cell patterning developed over the last decade has proven to be an efficient method for generating multicellular organizations with well controlled cell-cell interactions in 3D tissue scaffolds. An early method developed by Tan and Desai, for example, utilized microfluidics-assisted patterning to generate a biomimetic arterial structure formed by three different layers of cells (endothelial cells, smooth muscle cells, and fibroblasts) to mimic the 3-tunic structure found in vivo (Figure 15a). ${ }^{[299]}$ In their method, hydrogel precursor solutions containing different types of cells were sequentially injected into a microfluidic channel, which were then subjected to gelation inside the microfluidic channel before another hydrogel layer was delivered onto the existing hydrogel layers. By controlling the flow rate of hydrogel precursor solutions, the initial thickness of each hydrogel layer could be controlled. Instead of using microfluidic channels as templates for the layer-by-layer additive cell patterning, Tsang and colleagues also achieved the same goal using photopatterning of cell-laden PEG hydrogels to mimic a $3 \mathrm{D}$ hepatic tissue construct. ${ }^{[300]}$

In a more recent study, Albrecht and colleagues achieved a precise control over 3D multicellular patterning using dielectrophoretic (DEP) force-based cell manipulation in a layer-by-layer fashion (Figure 15b). ${ }^{[301]}$ DEP forces arise when polarizable a

By microfluidics

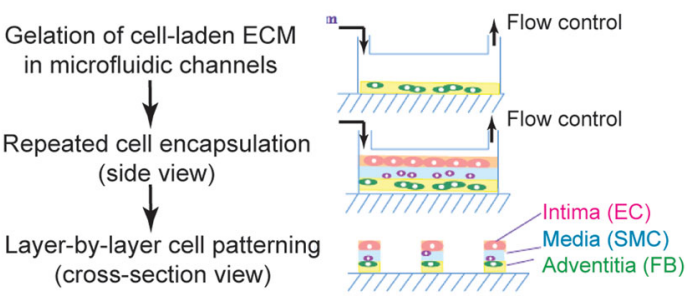

b

By DEP force

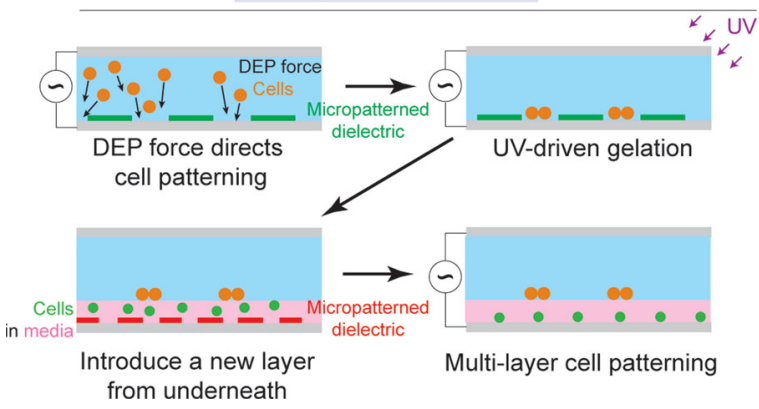

Figure 15. Layer-by-layer cell patterning for 3D tissue scaffolds. (a) Schematic of microfluidic-assisted layer-by-layer 3D cell patterning. ${ }^{[299]}$ A triple-layer cell-laden structure mimicking the blood vessel wall was created. Reproduced with permission. ${ }^{[299]}$ Copyright 2004, Elsevier. (b) Schematic of layer-by-layer cell patterning using dielectrophoretic (DEP) force. ${ }^{[301]}$ See text for detailed descriptions.

particles are subjected to a non-uniform electric field and have been widely used for dynamic manipulations of biological objects including cells. Albrecht and colleagues developed a DEP cell patterning (DCP) chamber that sandwiched uncrosslinked cell-laden prepolymer solution between two conductive indium tin oxide (ITO)-coated glass slides. On the bottom slide serving as an electrode, a thin micropatterned layer of insulating material - photoepoxy (SU-8) - masked most of the conductive surface to form micropatterned "electrodes" in all remaining un-masked areas. An alternating current (a.c.) bias applied across the top and bottom surfaces of the chamber produced a spatially non-uniform electric field that was strongest near the un-masked "electrodes" within the dielectric layer. Thus, DEP forces drove cells toward locations of highest electric field strength, i.e., pushing them toward the center of each exposed area of the bottom "electrode" slide. After cell patterning, UV light exposure through the transparent chamber covalently crosslinked the hydrogel, therefore entrapping the micropatterned cells as a thin layer within the gel. One might lift the hydrogel layer off the bottom slide before repeating the process to add a new hydrogel layer containing micropatterned cells from underneath. The DEP force-based layer-by-layer cell patterning method developed by Albrecht and colleagues is compatible with a wide variety of cell types and synthetic and natural ECMs. Furthermore, it allows well controlled studies of cellcell interactions in 3D microenvironments, which was indeed explored by the authors to show that microscale tissue organization regulates bovine articular chondrocyte biosynthesis. 

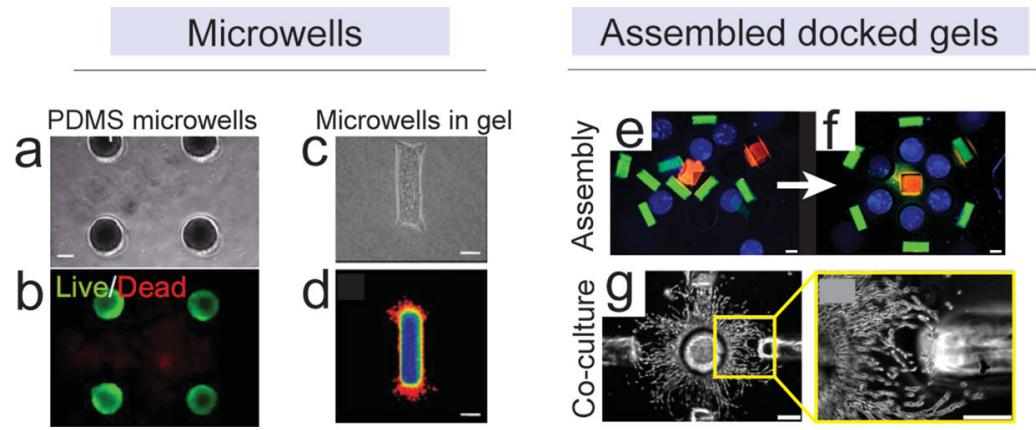

\section{D orthogonal photo-patterning}

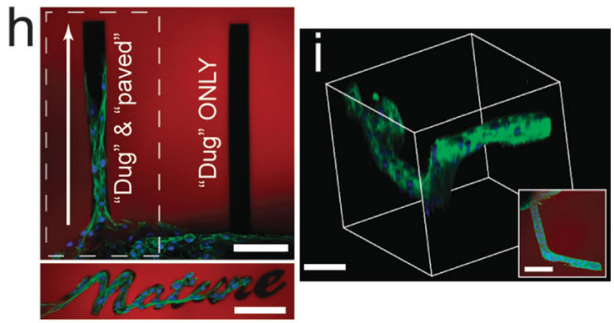

Figure 16. Other microengineering methods for fabricating 3D tissue scaffolds. (a-b) Phase contrast and fluorescent images of embryoid bodies cultured in PDMS microwells. ${ }^{[302]}$ Live/dead-staining was performed using calcein AM (green) and ethidium homodimer (red). Reproduced with permission. ${ }^{[302]}$ Copyright 2009, United States National Academy of Sciences. (c-d) Phase contrast image and frequency map of mammary epithelial

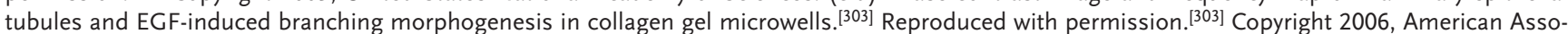
ciation for the Advancement of Science (e-f) Docked gelatin methacrylate (GelMA) hydrogels before and after the assembly. ${ }^{\left[{ }^{[05]}\right.}$ Different fluorescent molecules were used to mark docked gels with different shapes. (g) Confocal micrographs showing MSCs (in central gel) migrating toward neighboring gels containing co-cultured endothelial cells. ${ }^{[305]}$ Reproduced with permission. ${ }^{[305]}$ Copyright 2013, United States National Academy of Sciences. (h-i) Fluorescent images of guided 3D collective cell migration along a microtrack "dug" and "paved" by orthogonal photo-patterning. ${ }^{[134]}$ Cells did not migration along the microtrack without biochemical functionalization with RGD peptides. Reproduced with permission. ${ }^{[134]}$ Copyright 2011 , Nature Publishing Group.

Other Microengineering Methods for 3D Tissue Scaffolds. In addition to the microengineering methods discussed in the previous sessions, there are other non-traditional methods for generating microscale 3D tissue scaffolds, including molded microwell structures for controlling cell cluster size and shape, self-assembling hydrogel blocks, and cytocompatible photochemistries for dynamic 3D patterning of hydrogels. For example, microwells generated in PDMS or hydrogels using soft lithography were successfully applied to confine the size of embryoid bodies (EBs) formed by human ESCs. Using this hydrogel microwell strategy, Hwang and colleagues observed that cardiogenesis was enhanced in larger EBs, and in contrast, endothelial cell differentiation was increased in smaller EBs, and such EB-size regulated human ESC differentiation might involve WNT signaling (Figure 16a,b). ${ }^{[302]}$ The 3D confinement effect for cells seeded in 3D microwells was also exploited in another recent study to examine how actin CSK assembly and metabolism were different for single fibroblasts seeded in 3D microwells or on patterned 2D adhesive islands. ${ }^{[278]}$ In another notable study using molded microwell structures, Nelson and colleagues developed a powerful assay for studying mammary epithelial branching morphogenesis by embedding mouse mammary epithelial cells in cavities of collagen gel generated by molding unpolymerized collagen I around a patterned PDMS stamp (Figure 16c,d). ${ }^{[303]}$ Embedded epithelial cells formed hollow tubules conforming to the size and shape of the collagen cavities. Notably, subsequent branching morphogenesis of mammary epithelial cells were found to be determined by the geometry of tubules, correlated with local concentration profiles of autocrine inhibitory morphogens, such as transforming growth factor- $\beta$ (TGF- $\beta$ ), suggesting the involvement of TGF- $\beta$ in regulating branching morphogenesis of mammary epithelial cells.

Instead of fabricating a monolithic 3D tissue construct, $\mathrm{Du}$ and colleagues developed a bottom-up approach to direct the assembly of cell-laden microscale hydrogels to generate 3D tissue constructs with tunable architecture and complexity. ${ }^{[304]}$
The microtissue assembly process developed by $\mathrm{Du}$ and colleagues was driven by the tendency of multiphase liquid-liquid systems to minimize the surface area and the resulting surface free energy between the phases. In this work, Du and colleagues successfully demonstrated that shape-controlled microgels could spontaneously assemble within multiphase reactor systems into predetermined geometric configurations before a secondary cross-linking reaction to form multicomponent or multicellular 3D cell-laden constructs. In a more recent study, Eng and colleagues developed a similar strategy for multicomponent assembly of cell-laden constructs using geometrically docked hydrogel shapes (Figure 16e). ${ }^{[305]}$ In this method, microscale 3D hydrogels were shape-coded for their biological and physical properties and docked by iterative sedimentation into shape-matching hydrogel templates (Figure 16f). Furthermore, this technique was used to form radially organized 3D patterns to study sprouting and the homing of MSCs to ECs, by using diffusive signaling patterns to dictate spatial orientation of cell migration (Figure $16 \mathrm{~g}$ ). Compatible with a wide variety of hydrogel geometries and cell types, the simple but robust method demonstrated by Eng and colleagues could potentially provide a comprehensive approach to the assembly of 3D cell environment for studying cell-ECM and cell-cell interactions and their implications in disease mechanisms, tissue engineering and drug development.

Using cytocompatible photo-chemistries for dynamic 3D patterning of hydrogels is another powerful approach demonstrated for generating 3D tissue scaffolds in situ with a submicron resolution in the presence of live cells (see more discussions in section 2.1.3). In the notable study by DeForest and colleagues using photodegradable PEG-based hydrogels, an orthogonal photo-patterning of hydrogel mechanics and biochemistry was shown possible to guide pre-confined 3T3 fibroblasts to migrate collectively along a microscale track (or "dug") in the UV-degradable PEG hydrogel that was further functionalized using UV light (or "paved") with cell adhesion motifs (Figure 16h,i). ${ }^{[134]}$ 


\section{Integrated Multiparametric Functional Biomaterials: A New Frontier}

As discussed above, in the last two decades, many achievements have been made in fabricating functional biomaterials with different micro/nanoscale cues mimicking individual aspects of the in vivo cell microenvironment. These functional biomaterials have greatly facilitated cell biology studies for human health and diseases and inspired engineers, materials scientists and biologists to collaborate closely. From the perspective of fundamental science, these micro/nanoengineered functional biomaterials have unveiled novel mechanosensitive cellular behaviors in response to individual extracellular biophysical cues, and they have also been instrumental for providing insights of underlying molecular and cellular mechanisms for cell-material interactions by interweaving micro/ nanoengineering and materials science with cell biology. However, it is still unclear how individual extracellular biophysical cues may contribute to cellular behaviors in a physiological-like microenvironment, wherein multiple extracellular signals work in synergy or in competition with each other. Thus, the connection between mechanobiology and human development and diseases remains an enticing idea that requires solid scientific foundation. From the perspective of translational science, the advent of mechanobiology has provided new ideas and methodologies into the fields of biomaterial design, tissue engineering and regenerative medicine. For example, recent success in regulating behaviors of human pluripotent stem cells using extracellular biophysical cues has opened a promising new avenue for stem cell-based regenerative therapy and personalized medicine. However, a full promise of cell mechanobiology to regenerative medicine is still far from its delivery, as recapitulation of in vivo-like complex cellular functions observed at tissue and organ levels is still challenging due to the sophisticated dynamic interplays between different microenvironmental factors. Therefore, from both perspectives above, the new frontier of mechanobiology lies in the integration of micro/nanoengineered functional biomaterials for future innovations of multiparametric extracellular environment to study the fundamental roles of biophysical cues in physiologically and pathologically relevant contexts as well as to elicit complex tissue/organ functions with in vitro cell culture models answering to biomedical demands.

In the following two sections, we review recent progress integrating micro/nanoengineered functional biomaterials to develop miniaturized biological and biomimetic systems for dynamic multiparametric microenvironmental control of emergent and integrated cellular behaviors. Specifically we focus on two emerging topics that we believe can serve as both foundations and inspirations for future works in this exciting field: microengineered physiological biomimicry using the "organon-a-chip”, a promising direction for next-generation regenerative medicine and drug discovery; and microengineered cancer metastasis models, which exemplify the significance of engineered multiparametric cell culture environment to elucidate the fundamental connection between extracellular biophysical microenvironment and tissue/organ-level human developmental and disease processes.

\subsection{Microengineered Physiological Biomimicry: "Organ-on-a-Chip"}

Historically in vitro tissue culture models have been a standard as substitutes for costly and time-consuming animal models for drug discovery and toxicity screening. In the last 20 years, the rapid advancement of micro/nanoengineering and biomaterials science has delivered unprecedented power for precise, spatiotemporal control of individual aspects of the in vitro cell microenvironment. Successful it was for regulating single- and multi-cell level mechanoresponsive behaviors and functions; however, the effort of using in vitro monoparametric microenvironment for reconstituting in vivo physiologically-relevant cell and tissue functions was mostly unsatisfactory. Cells in vivo living in 3D ECM are subject to a multiparametric organspecific microenvironment, including ECM topography, matrix mechanics, cell-cell interactions, external mechanical forces, etc. It suggests that integration of different micro/nanoengineered extracellular cues within an in vitro microenvironment may be a prerequisite for engineered physiological biomimicry. ${ }^{[33,34]}$ Recently, the greatly advanced and widely adapted micro/nanofabrication techniques and biomaterials research have culminated in successful developments of integrated onchip cell culture systems reconstituting in vivo-like organ-level functions of lungs, ${ }^{[306,307]}$ intestines ${ }^{[308]}$ and kidneys, ${ }^{[309-311]}$ as well as many other "organ-on-a-chip" models that succeed in mimicking tissue-level functions of corneal, ${ }^{[312]}$ cardiac muscle, ${ }^{[313]}$ neural network, ${ }^{[314]}$ bile canaliculi[ ${ }^{[315]}$ and vascular

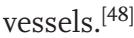

The integrative and multiparametric nature of in vivo cell microenvironment should include the following critical characteristics that are indispensable for recapitulating specific organ/ tissue functions and also serve as independent functional units in the development of integrated organ-on-a-chip models (Figure 17a-g). . $^{[3,34]}$

Tissue/chip-Scale Regulation of Chemical Environment. Local extracellular biochemical cues play an important role in tissue formation, function and maintenance, such as wnt proteins for embryo development, ${ }^{[165]}$ VEGF for angiogenesis ${ }^{[285]}$ and TGF$\beta$ for mammary branching morphogenesis. ${ }^{[303]}$ Tissues and organs located distantly from each other can also communicate effectively via circulating soluble factors. These observations strongly support the necessity for an in vivo-like biochemical microenvironment that should be specifically developed for integration into organ-on-a-chip models. One way of achieving this is to use microfluidic gradient generators to generate stable chemical gradients that are isolated from shear flow. ${ }^{[40]}$ In a recent study by Cimetta and colleagues, a stable wnt3a gradient has been achieved for long-term cell culture using a microfluidic design where source and sink microfluidic channels determines boundary concentrations of wnt3a while capillary channels connecting source and sink microfluidic channels maintain chemical gradients in central cell culture areas and protect cells from shear flow (Figure 17a). ${ }^{[316]}$ Similar microfluidic gradient generators are suitable for controlling biochemical microenvironment for organ/tissue models ${ }^{[48]}$ as well as single cells. ${ }^{[317]}$

The nature of in vivo biochemical communications between different organs is highly autonomous. Thus, it is difficult to 


\section{Critical functional elements \\ Tissue/chip-scale regulation of chemical gradient}

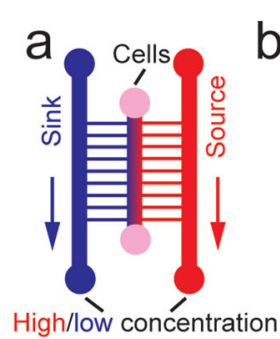

b

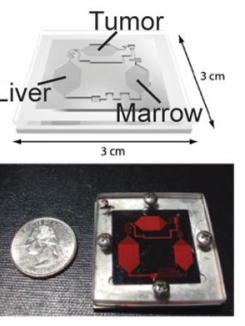

Barrier function and shear flow

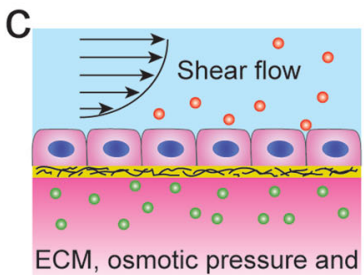

segregated chemical cues
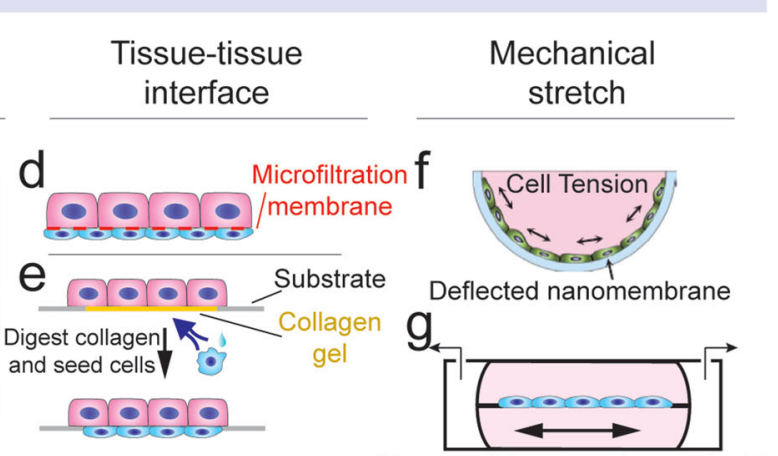

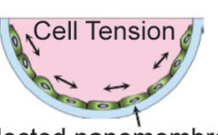

Deflected nanomembrane g.

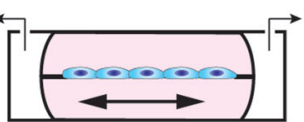

Vacuum-driven membrane stretch

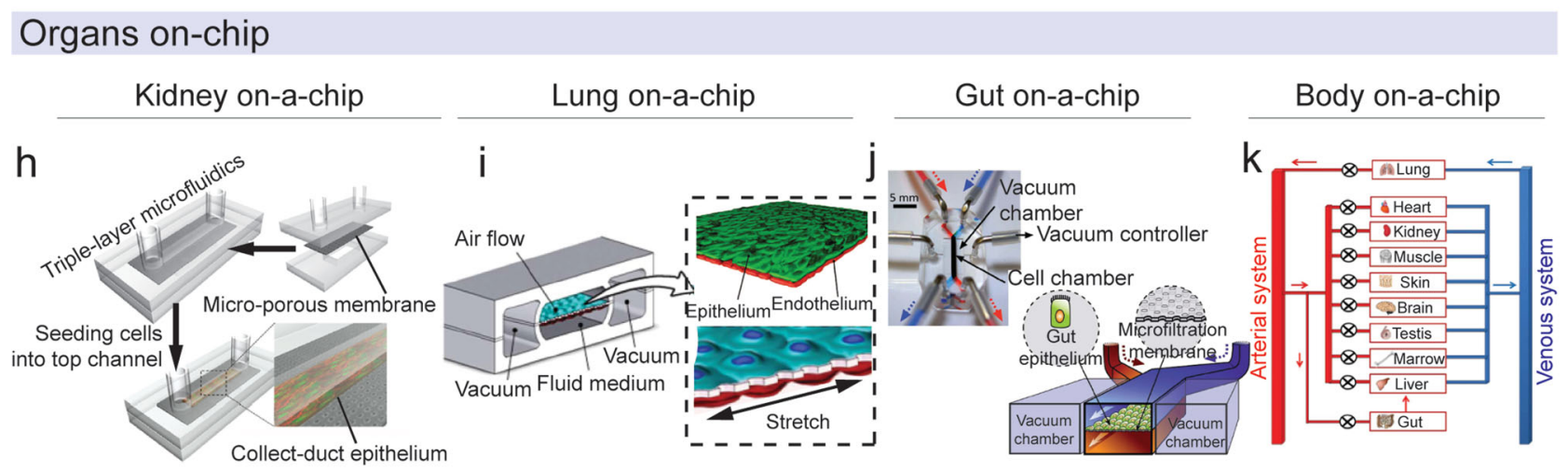

Figure 17. Integrating an in vivo-like multiparametric microenvironment for building in vitro organ models. (a) Schematic of a three-channel microfluidic design for creating long-term stable biochemical gradient. ${ }^{[36]}$ (b) Schematic (top) and picture (bottom) of a microfluidic design for coupling different cell culture modules for mimicking the autologous biochemical communications between different tissues/organs. ${ }^{[318]}$ It was used for studying the toxicity of anti-cancer drugs in the presence of metabolism by liver. Reproduced with permission. ${ }^{[318]}$ Copyright 2009, Royal Society of Chemistry. (c) Schematic of the barrier function of epithelia or endothelia, where the shear stress is present at the lumen side and the apical-basal asymmetry of ECM structure, osmotic pressure and biochemical cues all contribute to the function of epithelial or endothelia. ${ }^{[310]}$ (d) Schematic of epithelial-endothelial tissue coupling achieved via cell seeding on both sides of a microfiltration membrane. ${ }^{[306]}$ (e) Schematic of epithelial/endothelial cell-stromal cell coupling achieved via digesting sacrificial collagen gel from the backside. ${ }^{[12]}(\mathrm{f})$ Schematic of generating lateral mechanical stretch to a cell monolayer by out-of-plane nanomembrane deflection. ${ }^{[322]}$ (g) Schematic of generating in-plane mechanical stretch to a cell monolayer using vacuum driven sidewall-deflection in microfluidic channels. ${ }^{[306]}$ (h) Schematic of an on-chip cell culture model mimicking the function of renal tubules. ${ }^{[310]} \mathrm{A}$ monolayer of kidney tubule epithelial cells was cultured on a micro-porous membrane separating the top and bottom channels in a triple-layer microfluidic device. It enabled simultaneous modulation of multiple microenvironmental parameters for studying their effects on the morphology, polarity and transportation functions of renal epithelium, mimicking an organ-level function of the collecting duct. Reproduced with permission. ${ }^{310]}$ Copyright 2011, Royal Society of Chemistry. (i) Schematic of an on-chip cell culture model mimicking the function of lung. ${ }^{[306]}$ As the key structure, alveolar epithelium and vascular endothelium were conjugated to each other via a microporous membrane. The microfluidic design enabled differential media, shear stress and cyclic stretch applied simultaneously as key microenvironment mechanical stimuli for reconstituting organ-level functions of the lung. Reproduced with permission. ${ }^{[306]}$ Copyright 2010, American Association for the Advancement of Science (j) Schematic (right) and picture (left) of an on-chip cell culture model mimicking the function of intestine ${ }^{\left[{ }^{[08]}\right.}$ As the key structure, intestine epithelial monolayer was cultured on a microfiltration membrane. The microfluidics was employed to apply shear stress, fluid medium as well as peristaltic stretch, mimicking the micromechanical environment in the intestine. Reproduced with permission. ${ }^{[308]}$ Copyright 2012, Royal Society of Chemistry. (k) Schematic illustrating the concept of human body on-achip. ${ }^{[34]}$ By interconnecting different in vitro organ culture modules with a circulatory system, it could potentially lead to an autonomous on-chip model mimicking the inter-regulation among organ functions. Reproduced with permission. ${ }^{[34]}$ Copyright 2012, Royal Society of Chemistry.

recapitulate such cell-mediated biochemical communications using external means in a predictable manner. To mimic autonomous communications between different organs, Shuler and colleagues proposed to integrate different microtissue modules into the same chip to establish built-in biochemical coupling between the "tissues", which could have a significant effect on the drug toxicity assay outcome (Figure 17b). ${ }^{[318-320]}$ One notable aspect of their "organ-on-a-chip" design was the incorporated hepatic module for studying metabolism-dependent cytotoxicity of anti-cancer drugs. ${ }^{[318]}$ Using a similar approach, Imura and colleagues developed an intestine-liver-breast cancer model to mimic the influence of ingestion (from intestine microtissue) and hepatic metabolism (from liver microtissue) on the efficacy of anti-cancer drug targeting MCF-7 cancer cells. ${ }^{[321]}$ Together, this type of autonomous tissue-scale microfluidic regulation of chemical microenvironment is useful for reconstituting sophisticated tissue/organ models with multi-organ interactions. ${ }^{[34,320]}$

Barrier Function and Shear Flow. The barrier function is an important hallmark for epithelia and endothelia exhibiting structural and functional polarities (Figure 17c). Such structural 
and functional polarities in epithelial and endothelial cells, which are determined by several types of cell-cell interactions, including tight and adherens junctions, are critical for their barrier functions to control bi-directional passage of materials and transit of blood cells into and out of the bloodstream. Furthermore, apical tight junctions help maintain separated and polarized microenvironments for apical and basal cell membranes, of which the former directly experiences blood-borne or air-borne signals, whereas the latter is exposed to ECM with different sets of biophysical and biochemical cues. To reconstitute such polarized cellular features, a common strategy is to employ a triple-layer microfluidic structure with a semi-permeable membrane suspended in the middle between two microfluidic channels. ${ }^{[306,308,309]}$ After cells form a coherent monolayer on the membrane, both biophysical and biochemical microenvironmental parameters such as flow rate, cytokine/ hormone/pathogen concentrations, and osmotic pressure can be independently adjusted in the top and bottom microfluidic channels (Figure 17c). An alternative way to recapitulate barrier function and shear flow in vitro is to use the microfluidics-ingel biomaterials reviewed in Section 2.3, in which fluidic conditions for lumen cell monolayer are controlled via microfluidics while the physiochemical properties of ECM are regulated by gel crosslinking and bulk perfusion ${ }^{[285]}$ or by diffusion from a nearby source microchannel. ${ }^{[286]}$

Tissue-Tissue Interface. The structural conjunction between adjacent tissues within an organ has long been considered vital for organ-level functions in vivo, which rely on coordination and functional interactions between tissues, e.g., the alveolar epithelium and vascular endothelium in the lung. A common strategy used in "organ-on-a-chip" designs to mimic tissuetissue interfaces is to seed different types of cells on both sides of a thin microfiltration membrane - one monolayer on each side (Figure 17d). ${ }^{[306]}$ In such a sandwiched configuration, the microfiltration membrane serves as a support for cell monolayers while simultaneously providing free conducts for direct (cell-cell contact) and indirect (autocrine and paracrine signaling) communications between adjacent cell types. To achieve a more natural tissue-tissue interface, Puleo and colleagues developed a backside digestion method for constructing a corneal tissue model (Figure 17e). ${ }^{[312]}$ In their method, epithelial cells were first seeded onto a film of collagen gel to form an epithelium monolayer which deposited their own basement membrane. The collagen gel underneath was digested prior to a monolayer of stromal cells seeded from the backside of the collagen film. It is important to note that, in many "organ-ona-chip" designs with tissue interfaces other than the epithelialendothelial interface, e.g., EC-VSMC conjunction, at least one side of the tissue interface will be cell-embedding 3D ECM rather than a cell monolayer. For such tissue interfaces, the aforementioned methods can be adapted accordingly.

Mechanical Stretch. Many tissues in vivo are subject to different modes of mechanical stretches, e.g., blood pressure regulating stretches of vascular cells, breathing of the lung to stretch alveolar cells, and peristaltic motions of intestine cells. Establishing physiological-like mechanical stretches is considered especially critical for recapitulating functions of these tissues. In situ cell stretch has so far been applied to cultured cells via either nanomembrane deflection ${ }^{[322]}$ (Figure 17f) or in-plane membrane stretching ${ }^{[306,308]}$ (Figure $17 \mathrm{~g}$ ), both of which can be well controlled. While both methods are effective, the latter is more compatible with microscopy imaging.

In addition to the major characteristics for "organ-on-a-chip" designs discussed above, the significance of other less general microenvironmental factors has been demonstrated in "organon-a-chip" designs, such as electrical stimulations for cardiac muscle $e^{[313]}$ and neural network ${ }^{[314]}$ and controlling microtissue geometries for bile canaliculi models. ${ }^{[315]}$

Many "organ-on-a-chip" models have been successfully developed in recent years with different levels of success in recapitulating in vivo-like tissue/organ-level functions. Here we focus on discussing a few most prominent studies, in the hope that such a focused discussion will facilitate understanding the steps and criteria for successfully integrating and evaluating an organ-on-a-chip model, respectively. In the "kidney-ona-chip" model recently developed by Jang and colleagues, the major structural feature of renal tubule epithelia was mimicked by using a triple-layer microfluidics (Figure 17h). ${ }^{[309-311]}$ In addition, microenvironmental factors such as lumen fluid shear stress, basal biochemical stimulation and transepithelial osmotic gradient were successfully incorporated into the model. As benchmarks of physiological-like tissue functions, the renal tubule epithelia generated in this model exhibited aquaporin 2 (AQP2) at the apical cell membrane, a columnar cell morphology and formation of cilia. Furthermore, the "kidney-on-a-chip" model recapitulated the AQP2-apical membrane translocation and resultant water trafficking under basal stimulations of arginine vasopressin (AVP) and transepithelial osmotic gradients, known to promote water uptake in vivo. The "kidney-on-a-chip" model also mimicked successfully in vivo uptake of albumin and glucose. All together, the "kidneyon-a-chip" device reconstituted critical features of renal tubules within an in vitro multiparametric model designed to mimic key kidney tubule structures and functions. The "kidney-on-achip" model was recently applied to study renal toxicity using primary human kidney cells..$^{[311]}$

In the "lung-on-a-chip" model recently developed by Huh and colleagues, key structural features of the lung, i.e., the alveolar epithelium-vascular endothelium conjunction, were recapitulated by using a stretchable microfiltration membrane with each of the two sides of the membrane seeded with lung epithelial and endothelial cells (Figure 17i). ${ }^{[306,307]}$ The microfiltration membrane was cyclically stretched to apply external forces to the cells. Furthermore, the membrane was integrated into a triple-layer microfluidic device to incorporate other important microenvironmental cues relevant to the lung, e.g., air and liquid shear flow at the epithelial and endothelial apical surfaces, respectively, circulating immune cells (neutrophils) in contact with endothelia, and pulmonary stimuli such as TNF- $\alpha$, bacteria (E. coli) and nanoparticles. Tight junction integrity and barrier functions of the epithelial and endothelial sheets were promoted in such a highly integrated lung model. The "lungon-a-chip" model also recapitulated crucial in vivo-like organlevel inflammatory responses, e.g., increased expression of intercellular adhesion molecule 1 (ICAM-1) in endothelial cells, enhanced endothelial interactions with circulating neutrophils, transmigration and bacteria-hunting of neutrophils, as well as endothelial translocation of nanoparticles. The wide span of 
physiological-like behaviors of the "lung-on-a-chip" model demonstrates the promising applications of a well-designed multiparametric microenvironment for inducing organ-level functions even only with simplistic cell culture models in simple microfluidic environments.

Similar to the "lung-on-a-chip", a "gut-on-a-chip" model was recently developed by Kim and colleague (Figure 17j), ${ }^{[308]}$ which integrated major structural and microenvironmental features of intestine epithelial cells, such as an epithelial monolayer, a slow shear flow and a slow cyclic stretch mimicking peristaltic motions. The "gut-on-a-chip" model enabled reconstitutions of columnar cell morphology and epithelial barrier function, which was not possible if without shear and cell stretch. More importantly, the "gut-on-a-chip" model recapitulated in vivo-like expression of enzymes (e.g., aminopeptidases) in villi cells and physiological-like intestine microbial symbionts, critical for organ-level functions of the gut targeted in drug development.

Taking the three examples above together, and being supported as well by other recent organ-on-a-chip models, we can conclude three key steps (criteria) for integrating (evaluating) an in vivo-like organ-on-a-chip model, which should (1) reconstitute major structural features and tissue organizations of the target organ; (2) recapitulate major in vivo microenvironmental features associated with the target organ; (3) exhibit emergent organ-specific physiological-like functions that cannot be mimicked by conventional and microscale tissue culture models.

The ultimate goal for using microengineered physiological biomimicry to develop "organ-on-a-chip" models, as well as the body-on-a-chip concept (Figure 17k), is for translational studies like drug screening. This functional goal of the "organ-on-a-chip" research has been successfully achieved in the "lung-on-a-chip" applied to study pulmonary edema for identifying potential new therapeutics. ${ }^{[307]}$ Furthermore, development of "organ-ona-chip" models also delivers versatile microscale platforms integrating micro/nanoscale functional biomaterials for studying mechanobiology in physiologically and pathologically relevant contexts. For example, using the "kidney-on-a-chip" model,
Jang and colleagues discovered that shear flow-dependent depolymerization and rearrangement of actin CSK were closely correlated with flow-sensitive AQP2 apical translocation. ${ }^{[310]}$ Using the "lung-on-a-chip" model, Huh and colleagues revealed a general importance of mechanical stretch in promoting inflammatory responses under stimulations such as nanoparticle and IL2. ${ }^{[307]}$

It is important to note that, however, the field of "organ-ona-chip" for microengineered physiological biomimicry is still in its infancy, and the existing organ-on-a-chip models have only demonstrated limited applications of the rich toolbox of micro/ nanoengineered biomaterials as they mostly mimic planar and tubular tissue/organ structures of a local nature but not yet solid organs with intricate 3-D internal structures, varying cellular and extracellular properties, or multitude of interactions between functional groups of cells at a global scale. Given the current advances in this field, together with the rich resources of micro/nanoengineered functional biomaterials, it is foreseeable that developing in vitro multiparametric microenvironments through integrating micro/nanoengineered biomaterials within an organ-on-chip configuration will certainly shed light on both fundamental organ-level mechanobiology studies and translational medicine in the near future.

\subsection{Integrated Microenvironment for Studying Cancer Metastasis In Vitro}

Tumor cells navigate a long transformative journey during metastasis while closely interacting with surrounding ECM and stromal cells (Figure 18a). ${ }^{[3,5,17,18]}$ At the site of primary tumor, molecular machineries of tumor cells for cell-ECM and cell-cell interactions alter gradually during tumor growth, transforming cancer cell phenotypes from epithelial-like to mesenchymallike. ${ }^{[323]}$ Attenuation of cell-cell adhesion in tumor cells during the epithelial-to-mesenchymal transition (EMT) initiates their invasion into the surrounding ECM. ${ }^{[323,324]}$ Previous studies
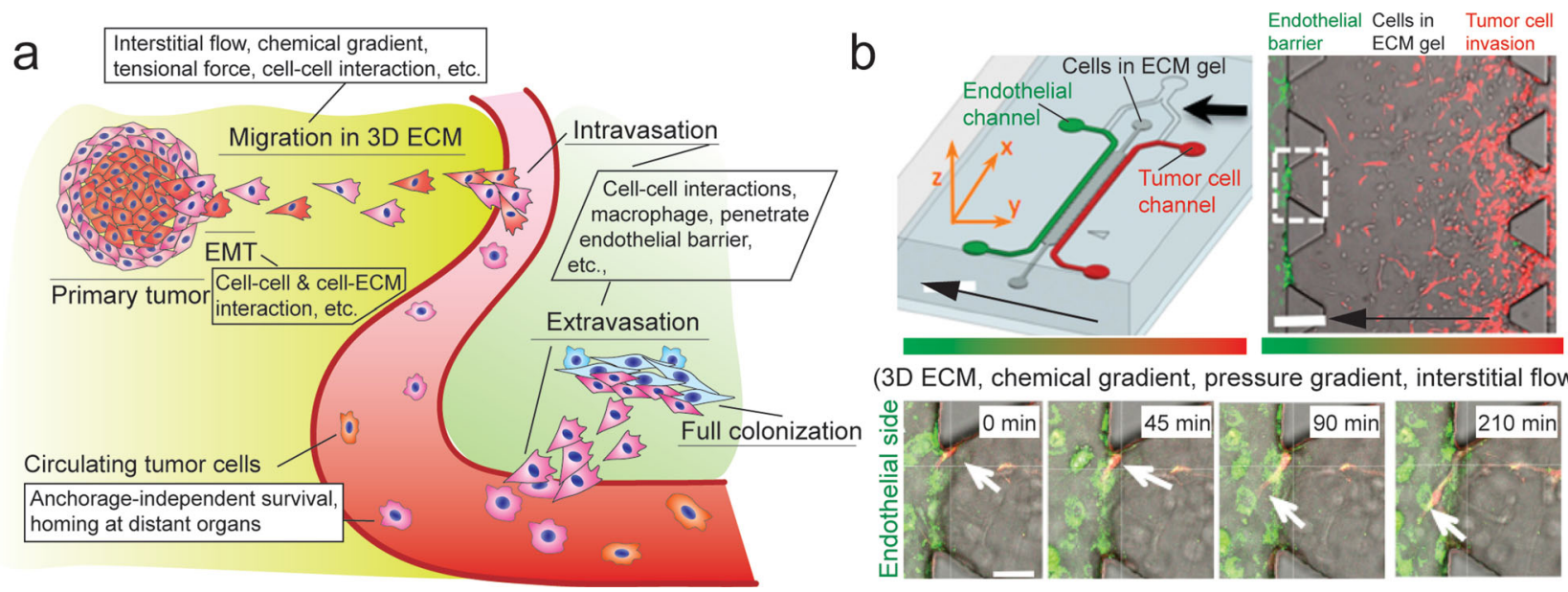

(3D ECM, chemical gradient, pressure gradient, interstitial flow)
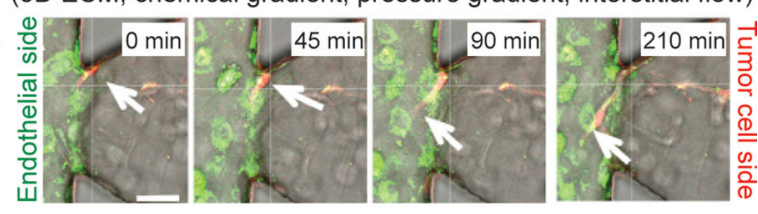

Figure 18. Integrated microenvironment for studying cancer metastasis in vitro. (a) Schematic of tumor cell-microenvironment interactions during the progression and metastasis of cancer. See text for detailed descriptions. (b) (upper left) Schematic of a representative gel-in-microfluidics assay for studying tumor cell intravasation. (upper right) Phase contrast image of the reconstituted tumor cell-endothelium interface. (bottom) Time-lapse confocal images showing a tumor cell invading and penetrating TNF- $\alpha$ stimulated endothelial monolayer. ${ }^{[344]}$ Reproduced with permission. ${ }^{[344]}$ Copyright 2012, United States National Academy of Sciences. 
have revealed that in addition to intrinsic gene regulatory networks, tumor cell migration (or invasion) is subject to regulations by multiple microenvironmental factors, such as physical confinement imposed by surrounding ECM, proteolytic matrix degradation, interstitial flow, chemokine gradient, and interactions with stromal cells like fibroblasts and macrophages. ${ }^{\text {[25-335] }}$ As another key step in cancer metastasis, tumor cell intravasation is also a complex process where biomechanical and biochemical interactions between tumor cells, endothelial cells and macrophages play an important role. ${ }^{[335,336]}$ Similarly, microenvironmental influences also hold true for tumor cell homing at distant sites, extravasation and metastatic colonization. ${ }^{[335,337]}$

In vivo study of cancer metastasis requires intravital imaging and multi-photon microscopy ${ }^{[54,336,338,339]}$ and thus is demanding on instruments and facilities. Therefore, researchers have been seeking to develop in vitro culture systems as an alternative to recapitulate and characterize tumor cell invasiveness and understand molecular/cellular mechanisms involved in each step of the cancer metastatic process. ${ }^{[340]}$ However, until recently, development of in vitro cancer metastatic models has been limited, since traditional 2D and 3D in vitro culture systems cannot recapitulate the complex dynamic multiparametric nature of in vivo interactions between tumor cells, surrounding ECM and stromal cells. Facilitated by recent progress in integrated micro/nanoengineered functional biomaterials, particularly by the developments of gel-in-microfluidics assays, there has been some exciting advancement for in vitro cancer metastatic models for mimicking the cancer metastatic process. ${ }^{[39,48,341]}$

Retrospectively speaking, gel-in-microfluidics assays can be considered functionally complementary to the microfluidic hydrogel biomaterials reviewed in Section 2.3. Fabricated using soft lithography, microfluidic hydrogel biomaterials have been proven particularly useful for fabrications of 3D tissue constructs and in vitro studies of vascular biology. ${ }^{[285,286]}$ However, owing to the monolithic nature of soft lithography, it is difficult to introduce spatially regulated complex biochemical cues, multiple tissue interfaces, or multiplex compartmentalized cell co-cultures within bulk hydrogels. Unfortunately, such a heterogeneous, spatially regulated multiparametric $3 \mathrm{D}$ microenvironment is a key for recapitulating the cancer metastatic process in vitro.

To establish in vivo-mimicking cancer metastasis models, gel-in-microfluidics assays have been recently developed (e.g., the 3-channel assay shown in Figure 18b), ${ }^{[39,48,341]}$ which enable convenient incorporations of: (1) the 3D microenvironment by using hydrogel matrix; ${ }^{[39]}$ (2) on-chip compartmentalization and positioning of cell-laden hydrogels using microfluidic chambers or photo-assisted patterning; ${ }^{[39,342]}$ (3) cell co-cultures using microfluidics-assisted patterning or compartmentalization; ${ }^{[34]}$ (4) reconstituted epithelial/endothelial lumen at hydrogel-microchannel interfaces; ${ }^{[344]}$ (5) spatially segregated delivery of biochemical cues and immune cells using intrinsic microfluidic designs and gel-encapsulation; ${ }^{[344]}$ and (6) longterm regulation of chemical and pressure gradients within hydrogels using microfluidics. ${ }^{[344,345]}$ Together, these features manifest the great potential of gel-in-microfluidics assays in recapitulating the cancer metastatic process in vitro.

Recently, Zervantonakis and colleagues developed an in vitro model for studying tumor cell intravasation. ${ }^{[34]}$ Using the gel-in-microfluidics assay shown in Figure 18b, Zervantonakis and colleagues generated in vivo-like endothelial barrier at the interface between hydrogel and microfluidic channels and a sub-lumen 3D gel matrix laden with tumor cells and macrophages. Due to paracrine signaling, encapsulated tumor cells migrated spontaneously toward the endothelial barrier. Alternatively, tumor cells seeded in a separate microfluidic channel migrated toward endothelium under the guidance of an EGF gradient. Interestingly, this gel-in-microfluidics tumor cell intravasation model recapitulated macrophage-assisted tumor cell intravasation observed in vivo, enabling in-depth studies of dynamical interactions between tumor cells, macrophages and endothelial cells. ${ }^{[336]}$ In subsequent mechanistic explorations, Zervantonakis and colleagues discovered that TNF- $\alpha$ compromised endothelial barrier function and resulted in rapid tumor cell intravasation without enhancing their intrinsic motility. Given that macrophages are known to secret TNF- $\alpha$ in the presence of tumor cells and potentiate their invasiveness, ${ }^{[346]}$ these results implied a mechanistic picture where macrophages assisted in tumor intravasation by regulating endothelium barrier function through secreting TNF- $\alpha .^{[336]}$

Using a similar in vivo-mimicking cancer metastasis model, Polacheck and colleagues studied tumor cell migration in 3D ECM under interstitial flow, ${ }^{[345]}$ which has long been recognized as important but the underlying molecular mechanism(s) is still under intense investigations. ${ }^{[326,327]}$ In their study, a pressure gradient was established across the central microfluidic channel area loaded with cell-laden hydrogels, and the rate of the interstitial flow was controlled by differential pressure from two side microchannels. This in vitro cancer metastasis model recapitulated post-EMT tumor cell migration behaviors within 3D matrix and revealed that a competition between a flow-driven CCR7 autologous chemotaxis and a flow-sensitive FA-related signaling process determined the overall migration direction of tumor cells under interstitial flow.

In complementary to the intravasation model, Chen and colleagues recently developed a tumor cell extravasation model using the gel-in-microfluidics assay. ${ }^{[347]}$ In this model, as the essential route for transporting circulating tumor cells and a barrier for their extravasation, a microvascular vessel network was engineered via self-assembly of vascular endothelial cells within a 3D fibrinogen hydrogel under a co-culture with human lung fibroblasts. ${ }^{[348]}$ After perfusing the vascular network with tumor cells, the dynamic process of tumor cell extravasation, including transendothelial migration, were directly visulized. Interestingly, this tumor cell extravasation model recapitulated the role of TNF- $\alpha$ in endothelial barrier function and transendothelial migration while revealing a correlation between the extravasation rate of tumor cells in vitro and their in vivo metastatic potentials. This in vitro tumor cell extravasation model also demonstrated that tumor cell clusters trapped within microvascular vessels had a greater chance for extravasation through transendothelial migration, supporting previous observations that aggregations of tumor cells in vivo with platelets and fibrin(ogen) might serve as an important mechanism for their survival in circulation and extravasation. ${ }^{[349]}$

Sung and colleagues also developed a Y-shaped gel-in-microfluidics assay to study the invasive phenotype transition in a breast cancer cell-mammary fibroblast co-culture system. ${ }^{[343]}$ 


\begin{tabular}{|c|c|c|}
\hline Fundamental mechano-sensitive & \multicolumn{2}{|c|}{ Fundamental Mechanobiology } \\
\hline Mechano-sensing mechanisms & $\begin{array}{l}\text { Quantitative role of cellular force } \\
\text { in mechanobiology }\end{array}$ & \\
\hline Mechanotransduction in cytoplasm & $\begin{array}{c}\text { Crosstalk between } \\
\text { mechano-sensing mechinaries }\end{array}$ & \\
\hline $\begin{array}{c}\text { Mechano-responsive gene } \\
\text { expression }\end{array}$ & $\begin{array}{l}\text { Crosstalk between } \\
\text { mechanotransduction pathways }\end{array}$ & $\begin{array}{l}\text { Mechanobiology in } \\
\text { physiology and pathology }\end{array}$ \\
\hline \multicolumn{3}{|c|}{ Mono-parametric $<$ Level of biomaterial integration } \\
\hline \multirow[t]{2}{*}{ Stem cell therapy } & $\begin{array}{c}\text { Synergistic effect in cell/tissue } \\
\text { engineering }\end{array}$ & $\begin{array}{l}\text { Tissue/organ model for } \\
\text { drug development }\end{array}$ \\
\hline & $\begin{array}{l}\text { Synergistic effect for sorting } \\
\text { subtle phenotypic heterogeneity }\end{array}$ & $\begin{array}{l}\text { Pathogenesis model for } \\
\text { finding better therapy target }\end{array}$ \\
\hline Translational Medicine & & $\begin{array}{l}\text { Integrated on-chip fast, robust } \\
\text { diagnosis at the point of care }\end{array}$ \\
\hline
\end{tabular}

Figure 19. Integrating micro/nanoengineered functional biomaterials for fundamental and translational sciences in future. While well-defined monoparametric biomaterials will continue contributing mostly to mechanistic studies of cell mechanobiology, integrated functional biomaterials are opening doors toward a vast scope of promising research topics on both fundamental mechanobiology and translational medicine in future.

By employing microfluidics-assisted intra-channel patterning and compartmentalization to regulate the interface between cancer cell-laden hydrogel and fibroblast-containing hydrogel, the authors observed that an intimate contact between tumor cells and stromal fibroblasts was required for enhancing the invasive phenotype of tumor cells. Drifka and colleagues recently adapted a similar idea in the development of an in vitro model for studying pancreatic ductal adenocarcinoma. ${ }^{[350]}$ By integrating a trilayer hydrogel micropatterning scheme in the microfluidic channel, they reconstituted the interface between pancreatic stellate cells (stromal cells) and pancreatic ductal adenocarcinoma cells (tumor cells, PANC-1). By modulating the composition and molecular architecture of the extracellular hydrogel materials (e.g., collagen I and hyaluronic acid), this in vitro model enabled regulations on both tumor cell-stromal cell interaction and cell-ECM interaction. Using disease-relevant human cells obtained from patients, they also studied the dosedependent response of this in vitro cancer model to anti-cancer agent paclitaxel. Via modulating the stromal microenvironment of cancer cells, these micropatterned gel-in-microfluidics assays could provide detailed understanding of tumor-stroma crosstalk and its role in regulating cancer metastasis.

In all, these pioneering studies have demonstrated the feasibility of building in vivo-like cancer metastasis models using integrated gel-in-microfluidics assays. Their developments and implementations have highlighted the unique contributions from these models for advancing our understanding of molecular and cellular mechanisms in cancer metastasis. In the future, additional levels of complexity can be integrated into the existing models, aiming at reconstituting an autonomous cancer metastasis model via a multi-module multiparametric microsystem on a chip. Future advancements in this field will certainly help reveal novel therapeutic targets for controlling cancer metastasis, which is of utmost importance for clinical treatment and management of cancer patients.

\section{Concluding Remarks and Outlook}

In the last two decades, the research fields of micro/nanoengineering and materials science have matured significantly to intersect and become connected for generating novel micro/ nanoengineered functional biomaterials for precise control of different aspects of the in vitro cell microenvironment at micro/nanoscale. These micro/nanoengineered functional biomaterials have revealed a large class of mechano-sensitive and -responsive cellular behaviors and enabled deterministic regulation of cellular behaviors using well-controlled in vitro cell microenvironment. From the perspective of fundamental biological science, many studies using synthetic micro/nanoengineered functional biomaterials have revealed important roles of mechanical forces and other microenvironmental biophysical cues in regulating embryo development, tissue morphogenesis, organogenesis, and human diseases such as cancer. From the perspective of translational medicine, these studies have facilitated knowledge advancement and technical innovations for tissue engineering and drug and toxicity screening. As a current trend, via integrations and coupling of micro/nanoengineered functional biomaterials, researchers have expanded in vitro cell microenvironment from monoparametric to multiparametric, in order to study many aspects of fundamental mechanobiology and translational medicine (Figure 19). This 
article presents a comprehensive review on recently developed micro/nanoengineered functional biomaterials for precise regulations of different aspects of the cell microenvironment and controlling dynamic cell-cell and cell-ECM interactions. We have further extended a discussion on the recent exciting trend where micro/nanoengineered biomaterials are integrated into miniaturized biological and biomimetic systems for dynamic multiparametric microenvironmental control of emergent and integrated cellular behaviors. We anticipate that in the near future, micro/nanoengineered functional biomaterials and their integration will provide new exciting opportunities for addressing challenges in fundamental mechanobiology studies and clinical and biomedical applications, such as regenerative medicine and drug and toxicity screening.

\section{Acknowledgements}

We acknowledge valuable comments and suggestions on the manuscript from members of the Integrated Biosystems and Biomechanics Laboratory. Work in Dr. Fu's lab is supported by the National Science Foundation (CMMI 1129611, CBET 1149401, ECCS 1231826, and CBET 1263889), the National Institute of Health (1R21HL114011), the American Heart Association (12SDG12180025), and the Department of Mechanical Engineering at the University of Michigan, Ann Arbor. Finally, we extend our apologies to all our colleagues in the field whose work we are unable to discuss formally because of space constraints.

Received: September 3, 2013

Revised: October 11, 2013

Published online: December 12, 2013

[1] D. Ingber, Ann. Med. 2003, 35, 564

[2] M. A. Wozniak, C. S. Chen, Nat. Rev. Mol. Cell Biol. 2009, 10, 34

[3] G. Gupta, J. Massagué, Cell. 2006, 127, 679.

[4] J. Joyce, J. Pollard, Nat. Rev. Cancer. 2009, 9, 239.

[5] P. Friedl, S. Alexander, Cell 2011, 147, 992.

[6] T. Mammoto, A. Mammoto, D. Ingber, Annu. Rev. Cell Dev. Biol. 2013, 29, 27.

[7] A. Folch, M. Toner, Annu. Rev. Biomed. Eng. 2000, 2, 227.

[8] G. M. Whitesides, E. Ostuni, S. Takayama, X. Y. Jiang, D. E. Ingber, Annu. Rev. Biomed. Eng. 2001, 3, 335.

[9] D.-H. Kim, P. Wong, J. Park, A. Levchenko, Y. Sun, Annu. Rev. Biomed. Eng. 2009, 11, 203.

[10] G. Underhill, P. Galie, C. Chen, S. Bhatia, Annu. Rev. Cell Dev. Biol. 2012, 28, 385

[11] D.-H. Kim, H. Lee, Y. Lee, J.-M. Nam, A. Levchenko, Adv. Mater. 2010, 22, 4551.

[12] P. Zorlutuna, N. Annabi, G. Camci-Unal, M. Nikkhah, J. Cha, J. Nichol, A. Manbachi, H. Bae, S. Chen, A. Khademhosseini, Adv. Mater. 2012, 24, 1782.

[13] A. Engler, S. Sen, H. Sweeney, D. Discher, Cell 2006, 126, 677.

[14] R. Tenney, D. Discher, Curr. Opin. Cell Biol. 2009, 21, 630.

[15] R. McBeath, D. M. Pirone, C. M. Nelson, K. Bhadriraju, C. S. Chen, Dev. Cell. 2004, 6, 483.

[16] D.-H. Kim, P. Provenzano, C. Smith, A. Levchenko, J. Cell Biol. 2012, 197, 351.

[17] D. Butcher, T. Alliston, V. Weaver, Nat. Rev. Cancer. 2009, 9, 108.

[18] D. Wirtz, K. Konstantopoulos, P. Searson, Nat. Rev. Cancer. 2011, $11,512$.

[19] C. S. Chen, J. Cell Sci. 2008, 121, 3285.

[20] D. Ingber, FASEB J. 2006, 20, 811 .
[21] D. Discher, P. Janmey, Y.-L. Wang, Science 2005, 310, 1139.

[22] A. Buxboim, I. Ivanovska, D. Discher, J. Cell Sci. 2010, 123, 297.

[23] A. Keung, S. Kumar, D. Schaffer, Annu. Rev. Cell Dev. Biol. 2010, 26, 533.

[24] J. Eyckmans, T. Boudou, X. Yu, C. Chen, Dev. Cell. 2011, 21, 35.

[25] J. Gasiorowski, C. Murphy, P. Nealey, Annu. Rev. Biomed. Eng. 2013, 15, 155

[26] F. Guilak, D. Cohen, B. Estes, J. Gimble, W. Liedtke, C. Chen, Cell Stem Cell. 2009, 5, 17.

[27] Kshitiz, D.-H. Kim, D. Beebe, A. Levchenko, Trends Biotechnol. 2011, 29, 399.

[28] S. Suresh, Acta Mater. 2007, 55, 3989.

[29] J. R. S. Newman, S. Ghaemmaghami, J. Ihmels, D. K. Breslow, M. Noble, J. L. DeRisi, J. S. Weissman, Nature. 2006, 441, 840.

[30] M. Y. Wu, A. K. Singh, Curr. Opin. Biotech. 2012, 23, 83.

[31] Q. H. Shi, L. D. Qin, W. Wei, F. Geng, R. Fan, Y. S. Shin, D. L. Guo, L. Hood, P. S. Mischel, J. R. Heath, Proc. Natl. Acad. Sci. U.S.A. 2012, 109, 419

[32] K. Willison, D. Klug, Curr. Opin. Biotech. 2013, 24, 745.

[33] D. Huh, G. Hamilton, D. Ingber, Trends Cell Biol. 2011, 21, 745.

[34] D. Huh, Y.-S. Torisawa, G. Hamilton, H. Kim, D. Ingber, Lab Chip. 2012, 12, 2156

[35] M. Schwartz, C. S. Chen, Science 2013, 339, 402.

[36] F. Pampaloni, E. Reynaud, E. Stelzer, Nat. Rev. Mol. Cell Biol. 2007, $8,839$.

[37] L. Griffith, M. Swartz, Nat. Rev. Mol. Cell Biol. 2006, 7, 211.

[38] D. Qin, Y. Xia, G. Whitesides, Nat. Protoc. 2010, 5, 491.

[39] Y. Shin, S. Han, J. Jeon, K. Yamamoto, I. Zervantonakis, R. Sudo, R. Kamm, S. Chung, Nat. Protoc. 2012, 7, 1247.

[40] S. Sia, G. Whitesides, Electrophoresis. 2003, 24, 3563.

[41] M. Théry, J. Cell Sci. 2010, 123, 4201.

[42] P. Kharkar, K. Kiick, A. Kloxin, Chem. Soc. Rev. 2013, In Press.

[43] C. Choi, M. Breckenridge, C. Chen, Trends Cell Biol. 2010, 20, 705.

[44] A. Khademhosseini, R. Langer, Biomaterials 2007, 28, 5087.

[45] C. M. Kirschner, K. S. Anseth, Acta Mater. 2013, 61, 931.

[46] A. Higuchi, Q.-D. Ling, Y. Chang, S.-T. Hsu, A. Umezawa, Chem. Rev. 2013, 113, 3297.

[47] M. Guvendiren, J. Burdick, Curr. Opin. Biotech. 2013, In Press.

[48] K. Wong, J. Chan, R. Kamm, J. Tien, Annu. Rev. Biomed. Eng. 2012, 14, 205.

[49] V. Vogel, M. Sheetz, Nat. Rev. Mol. Cell Biol. 2006, 7, 265.

[50] Y. B. Sun, C. S. Chen, J. P. Fu, Annu. Rev. Biophys. 2012, 41, 519.

[51] P. Janmey, R. Miller, J. Cell Sci. 2011, 124, 9.

[52] C. Hahn, M. Schwartz, Nat. Rev. Mol. Cell Biol. 2009, 10, 53.

[53] C. C. DuFort, M. J. Paszek, V. M. Weaver, Nat. Rev. Mol. Cell Biol. 2011, 12, 308.

[54] J. Condeelis, J. E. Segall, Nat. Rev. Cancer. 2003, 3, 921.

[55] Y. N. Xia, G. M. Whitesides, Annu. Rev. Mater. Sci. 1998, 28, 153.

[56] C. J. Hawker, T. P. Russell, MRS Bull. 2005, 30, 952.

[57] M. Morariu, N. Voicu, E. Schäffer, Z. Lin, T. Russell, U. Steiner, Nature Mater. 2003, 2, 48.

[58] D.-H. Kim, C.-H. Seo, K. Han, K. W. Kwon, A. Levchenko, K.-Y. Suh, Adv. Funct. Mater. 2009, 19, 1579.

[59] D.-H. Kim, K. Han, K. Gupta, K. Kwon, K.-Y. Suh, A. Levchenko, Biomaterials 2009, 30, 5433.

[60] T. W. Odom, C. J. Love, D. B. Wolfe, K. E. Paul, G. M. Whitesides, Langmuir 2002, 18, 5314.

[61] Y. Xia, E. Kim, X.-M. Zhao, J. A. Rogers, M. Prentiss, G. M. Whitesides, Science 1996, 273, 347.

[62] M. Nikkhah, F. Edalat, S. Manoucheri, A. Khademhosseini, Biomaterials 2012, 33, 5230.

[63] J. Hwang, S. Cho, J. Dang, E. Kwak, K. Song, J. Moon, M. Sung, Nature Nanotech. 2010, 5, 742.

[64] H. Unadkat, M. Hulsman, K. Cornelissen, B. Papenburg, R. Truckenmüller, A. Carpenter, M. Wessling, G. Post, M. Uetz, 
M. Reinders, D. Stamatialis, C. van Blitterswijk, J. de Boer, Proc. Natl. Acad. Sci. U.S.A. 2011, 108, 16565.

[65] J. Xie, S. Willerth, X. Li, M. Macewan, A. Rader, S. Sakiyama-Elbert, Y. Xia, Biomaterials 2009, 30, 354.

[66] G. Christopherson, H. Song, H.-Q. Mao, Biomaterials 2009, 30, 556.

[67] M. Schindler, I. Ahmed, J. Kamal, A. Nur-E-Kamal, T. Grafe, H. Young Chung, S. Meiners, Biomaterials 2005, 26, 5624.

[68] X. Xin, M. Hussain, J. Mao, Biomaterials 2007, 28, 316.

[69] Q. P. Pham, U. Sharma, A. G. Mikos, Tissue Eng. 2006, 12, 1197.

[70] M. Kharaziha, M. Nikkhah, S. R. Shin, N. Annabi, N. Masoumi, A. K. Gaharwar, G. Camci-Unal, A. Khademhosseini, Biomaterials 2013, 34, 6355.

[71] W. T. Liu, Y. Wei, X. H. Zhang, M. M. Xu, X. P. Yang, X. L. Deng, ACS Nano 2013, 7, 6928.

[72] B. Lanfer, U. Freudenberg, R. Zimmermann, D. Stamov, V. Körber, C. Werner, Biomaterials 2008, 29, 3888.

[73] N. Huang, J. Okogbaa, J. Lee, A. Jha, T. Zaitseva, M. Paukshto, J. Sun, N. Punjya, G. Fuller, J. Cooke, Biomaterials 2013, 34, 4038.

[74] S. Patel, K. Kurpinski, R. Quigley, H. Gao, B. Hsiao, M.-M. Poo, S. Li, Nano Lett. 2007, 7, 2122.

[75] M. Dalby, M. Riehle, H. Johnstone, S. Affrossman, A. Curtis, Biomaterials 2002, 23, 2945.

[76] M. Dalby, D. Giannaras, M. Riehle, N. Gadegaard, S. Affrossman, A. Curtis, Biomaterials 2004, 25, 77

[77] C. González-García, S. Sousa, D. Moratal, P. Rico, M. Salmerón-Sánchez, Colloid. Surface. B. 2010, 77, 181.

[78] P. Hanarp, D. Sutherland, J. Gold, B. Kasemo, Colloid. Surface. A. 2003, 214, 23

[79] M. Dalby, M. Riehle, D. Sutherland, H. Agheli, A. Curtis, Eur. J. Cell Biol. 2004, 83, 159.

[80] W. Chen, L. Villa-Diaz, Y. Sun, S. Weng, J. Kim, R. Lam, L. Han, R. Fan, P. Krebsbach, J. Fu, ACS Nano 2012, 6, 4094.

[81] W. Chen, Y. Sun, J. Fu, Small. 2013, 9, 81

[82] W. Chen, S. Weng, F. Zhang, S. Allen, X. Li, L. Bao, R. Lam, J. Macoska, S. Merajver, J. Fu, ACS Nano 2013, 7, 566.

[83] S. Oh, K. Brammer, Y. Li, D. Teng, A. Engler, S. Chien, S. Jin, Proc. Natl. Acad. Sci. U.S.A. 2009, 106, 2130

[84] V. Brunetti, G. Maiorano, L. Rizzello, B. Sorce, S. Sabella, R. Cingolani, P. Pompa, Proc. Natl. Acad. Sci. U.S.A. 2010, 107, 6264

[85] R. B. Dickinson, S. Guido, R. T. Tranquillo, Ann. Biomed. Eng. 1994, 22, 342.

[86] X. F. Walboomers, H. J. E. Croes, L. A. Ginsel, J. A. Jansen, J. Biomed. Mater. Res. 1999, 47, 204.

[87] A. Teixeira, G. Abrams, P. Bertics, C. Murphy, P. Nealey, J. Cell Sci. 2003, 116, 1881.

[88] S. W. Moore, P. Roca-Cusachs, M. P. Sheetz, Dev. Cell. 2010, 19, 194.

[89] M. Dalby, N. Gadegaard, R. Tare, A. Andar, M. Riehle, P. Herzyk, C. Wilkinson, R. Oreffo, Nature Mater. 2007, 6, 997

[90] R. McMurray, N. Gadegaard, P. Tsimbouri, K. Burgess, L. McNamara, R. Tare, K. Murawski, E. Kingham, R. Oreffo, M. Dalby, Nature Mater. 2011, 10, 637.

[91] J. Recknor, D. Sakaguchi, S. Mallapragada, Biomaterials 2006, 27, 4098.

[92] F. Pan, M. Zhang, G. Wu, Y. Lai, B. Greber, H. Schöler, L. Chi, Biomaterials 2013, In Press.

[93] S. Ankam, M. Suryana, L. Y. Chan, A. A. K. Moe, B. K. K. Teo, J. B. K. Law, M. P. Sheetz, H. Y. Low, E. K. F. Yim, Acta Biomater. 2013, 9, 4535

[94] P. M. Tsimbouri, R. J. McMurray, K. V. Burgess, E. V. Alakpa, P. M. Reynolds, K. Murawski, E. Kingham, R. O. C. Oreffo, N. Gadegaard, M. J. Dalby, ACS Nano 2012, 6, 10239
[95] D.-H. Kim, P. Kim, I. Song, J. Cha, S. Lee, B. Kim, K. Suh, Langmuir 2006, 22, 5419.

[96] D.-H. Kim, E. Lipke, P. Kim, R. Cheong, S. Thompson, M. Delannoy, K.-Y. Suh, L. Tung, A. Levchenko, Proc. Natl. Acad. Sci. U.S.A. 2010, 107, 565.

[97] B. K. Teo, S. Wong, C. Lim, T. Kung, C. Yap, Y. Ramgopal, L. Romer, E. Yim, ACS Nano 2013, In Press.

[98] K. Yang, K. Jung, E. Ko, J. Kim, K. Park, J. Kim, S.-W. Cho, ACS Appl. Mater. Interfaces. 2013, In Press.

[99] P. Kanchanawong, G. Shtengel, A. Pasapera, E. Ramko, M. Davidson, H. Hess, C. Waterman, Nature. 2010, 468, 580.

[100] S. Kumar, V. Weaver, Cancer Metast. Rev. 2009, 28, 113.

[101] Y. B. Sun, J. P. Fu, Integr. Biol. 2013, 5, 450.

[102] A. K. Harris, P. Wild, D. Stopak, Science 1980, 208, 177.

[103] M. Paszek, N. Zahir, K. Johnson, J. Lakins, G. Rozenberg, A. Gefen, C. Reinhart-King, S. Margulies, M. Dembo, D. Boettiger D. Hammer, V. Weaver, Cancer Cell. 2005, 8, 241.

[104] S. Chien, Am. J. Physiol. Heart Circ. Physiol. 2007, 292, H1209.

[105] J. Tan, J. Tien, D. Pirone, D. Gray, K. Bhadriraju, C. Chen, Proc Natl. Acad. Sci. U.S.A. 2003, 100, 1484.

[106] A. Saez, M. Ghibaudo, A. Buguin, P. Silberzan, B. Ladoux, Proc. Natl. Acad. Sci. U.S.A. 2007, 104, 8281.

[107] J. Fu, Y.-K. Wang, M. Yang, R. Desai, X. Yu, Z. Liu, C. Chen, Nat. Methods. 2010, 7, 733.

[108] M. Yang, J. Fu, Y.-K. Wang, R. Desai, C. Chen, Nat. Protoc. 2011, 6, 187.

[109] L. Trichet, J. Le Digabel, R. Hawkins, S. Vedula, M. Gupta, C. Ribrault, P. Hersen, R. Voituriez, B. Ladoux, Proc. Natl. Acad Sci. U.S.A. 2012, 109, 6933

[110] M. T. Yang, N. J. Sniadecki, C. S. Chen, Adv. Mater. 2007, 19, 3119.

[111] O. du Roure, A. Saez, A. Buguin, R. Austin, P. Chavrier, P. Silberzan, P. Siberzan, B. Ladoux, Proc. Natl. Acad. Sci. U.S.A 2005, 102, 2390

[112] Y. Sun, L.-T. Jiang, R. Okada, J. Fu, Langmuir 2012, 28, 10789

[113] S. Rahmouni, A. Lindner, F. Rechenmacher, S. Neubauer, T. Sobahi, H. Kessler, E. Cavalcanti-Adam, J. Spatz, Adv. Mater. 2013, In Press.

[114] S. Petronis, J. Gold, B. Kasemo, J. Micromech. Microeng. 2003, 13, 900.

[115] P. Kim, A. Epstein, M. Khan, L. Zarzar, D. Lipomi, G. Whitesides, J. Aizenberg, Nano Lett. 2012, 12, 527.

[116] K. A. Beningo, M. Dembo, I. Kaverina, J. V. Small, Y. L. Wang, J. Cell Biol. 2001, 153, 881

[117] E. M. Erb, K. Tangemann, B. Bohrmann, B. Muller, J. Engel, Biochemistry. 1997, 36, 7395

[118] I. Schoen, B. Pruitt, V. Vogel, Ann. Rev. Mater. Res. 2013, 43, 6.1.

[119] R. Pelham, Y. Wang, Proc. Natl. Acad. Sci. U.S.A. 1997, 94, 13661.

[120] A. Engler, M. Griffin, S. Sen, C. Bönnemann, H. Sweeney, D. Discher, J. Cell Biol. 2004, 166, 877

[121] M. Raab, J. Swift, P. Dingal, P. Shah, J.-W. Shin, D. Discher, J. Cell Biol. 2012, 199, 669

[122] Y. A. Miroshnikova, D. M. Jorgens, L. Spirio, M. Auer A. L. Sarang-Sieminski, V. M. Weaver, Phys. Biol. 2011, 8, 026013.

[123] J. Tse, A. Engler, Current Protocols in Cell Biology. 2010, Ch. 10.

[124] B. Trappmann, J. Gautrot, J. Connelly, D. Strange, Y. Li, M. Oyen, M. Cohen Stuart, H. Boehm, B. Li, V. Vogel, J. Spatz, F. Watt, W. Huck, Nature Mater. 2012, 11, 642.

[125] J. Ramón-Azcón, S. Ahadian, M. Estili, X. Liang, S. Ostrovidov, H. Kaji, H. Shiku, M. Ramalingam, K. Nakajima, Y. Sakka, A. Khademhosseini, T. Matsue, Adv. Mater. 2013, 25, 4028.

[126] S. Shin, B. Aghaei-Ghareh-Bolagh, T. Dang, S. Topkaya, X. Gao S. Yang, S. Jung, J. Oh, M. Dokmeci, X. Tang, A. Khademhosseini, Adv. Mater. 2013, In Press.

[127] C. Lo, H. Wang, M. Dembo, Y. Wang, Biophys. J. 2000, 79, 144.

[128] N. Zaari, P. Rajagopalan, S. K. Kim, A. J. Engler, J. Y. Wong, Adv. Mater. 2004, 16, 2137. 
[129] Y. Cheung, E. Azeloglu, D. Shiovitz, K. Costa, D. Seliktar, S. Sia, Angew. Chem. Int. Edit. 2009, 48, 7188.

[130] M. Guvendiren, J. Burdick, Nat. Commun. 2012, 3, 792.

[131] S. Khetan, M. Guvendiren, W. Legant, D. Cohen, C. Chen, J. Burdick, Nature Mater. 2013, 12, 458

[132] C. DeForest, B. Polizzotti, K. Anseth, Nature Mater. 2009, 8, 659.

[133] A. Kloxin, M. Tibbitt, K. Anseth, Nat. Protoc. 2010, 5, 1867.

[134] C. DeForest, K. Anseth, Nature Chem. 2011, 3, 925

[135] C. DeForest, K. Anseth, Angew. Chem. Int. Edit. 2012, 51, 1816.

[136] D. Aydin, I. Louban, N. Perschmann, J. Blummel, T. Lohmuller, E. A. Cavalcanti-Adam, T. L. Haas, H. Walczak, H. Kessler, R. Fiammengo, J. P. Spatz, Langmuir 2010, 26, 15472.

[137] D.-H. Kim, S. Khatau, Y. Feng, S. Walcott, S. Sun, G. Longmore, D. Wirtz, Sci. Rep. 2012, 2, 555.

[138] M. Prager-Khoutorsky, A. Lichtenstein, R. Krishnan, K. Rajendran, A. Mayo, Z. Kam, B. Geiger, A. D. Bershadsky, Nat. Cell Biol. 2011, $13,1457$.

[139] Y. B. Sun, L. G. Villa-Diaz, R. H. W. Lam, W. Q. Chen, P. H. Krebsbach, J. P. Fu, PLoS One. 2012, 7, e37178.

[140] T. Ulrich, E. de Juan Pardo, S. Kumar, Cancer Res. 2009, 69, 4167.

[141] A. Kostic, C. Lynch, M. Sheetz, PLoS One. 2009, 4, e6361.

[142] N. Huebsch, P. Arany, A. Mao, D. Shvartsman, O. Ali, S. Bencherif, J. Rivera-Feliciano, D. Mooney, Nature Mater. 2010, 9, 518.

[143] S. I. Fraley, Y. F. Feng, R. Krishnamurthy, D. H. Kim, A. Celedon, G. D. Longmore, D. Wirtz, Nat. Cell Biol. 2010, 12, 598.

[144] A. Banerjee, M. Arha, S. Choudhary, R. Ashton, S. Bhatia, D. Schaffer, R. Kane, Biomaterials 2009, 30, 4695.

[145] K. Saha, A. Keung, E. Irwin, Y. Li, L. Little, D. Schaffer, K. Healy, Biophys. J. 2008, 95, 4426.

[146] M. L. Gardel, I. C. Schneider, Y. Aratyn-Schaus, C. M. Waterman, Annu. Rev. Cell Dev. Biol. 2010, 26, 315.

[147] P. Roca-Cusachs, T. Iskratsch, M. Sheetz, J. Cell Sci. 2012, 125, 3025 .

[148] B. Geiger, J. P. Spatz, A. D. Bershadsky, Nat. Rev. Mol. Cell Biol. 2009, 10, 21.

[149] W. J. Guo, F. G. Giancotti, Nat. Rev. Mol. Cell Biol. 2004, 5, 816.

[150] M. A. Wozniak, R. Desai, P. A. Solski, C. J. Der, P. J. Keely, J. Cell Biol. 2003, 163, 583.

[151] D. J. Pan, Dev. Cell. 2010, 19, 491

[152] S. Dupont, L. Morsut, M. Aragona, E. Enzo, S. Giulitti, M. Cordenonsi, F. Zanconato, J. Le Digabel, M. Forcato, S. Bicciato, N. Elvassore, S. Piccolo, Nature. 2011, 474, 179.

[153] K. F. Harvey, X. M. Zhang, D. M. Thomas, Nat. Rev. Cancer. 2013, $13,246$.

[154] K. I. Wada, K. Itoga, T. Okano, S. Yonemura, H. Sasaki, Development. 2011, 138, 3907.

[155] M. Aragona, T. Panciera, A. Manfrin, S. Giulitti, F. Michielin, N. Elvassore, S. Dupont, S. Piccolo, Cell 2013, 154, 1047.

[156] A. Ulbricht, F. J. Eppler, V. E. Tapia, P. F. M. van der Ven, N. Hampe, N. Hersch, P. Vakeel, D. Stadel, A. Haas, P. Saftig, C. Behrends, D. O. Furst, R. Volkmer, B. Hoffmann, W. Kolanus, J. Hohfeld, Curr. Biol. 2013, 23, 430.

[157] J. Swift, I. L. Ivanovska, A. Buxboim, T. Harada, P. C. D. P. Dingal, J. Pinter, J. D. Pajerowski, K. R. Spinler, J. W. Shin, M. Tewari, F. Rehfeldt, D. W. Speicher, D. E. Discher, Science 2013, 341, 975.

[158] A. J. Keung, P. Asuri, S. Kumar, D. V. Schaffer, Integr. Biol. 2012, 4, 1049.

[159] Z. Li, Y. W. Gong, S. J. Sun, Y. Du, D. Y. Lu, X. F. Liu, M. Long, Biomaterials 2013, 34, 7616.

[160] J. Lee, A. Abdeen, D. Zhang, K. Kilian, Biomaterials 2013, In Press.

[161] J. Holst, S. Watson, M. Lord, S. Eamegdool, D. Bax, L. Nivison-Smith, A. Kondyurin, L. Ma, A. Oberhauser, A. Weiss, J. Rasko, Nat. Biotechnol. 2010, 28, 1123.

[162] C. Lee-Thedieck, N. Rauch, R. Fiammengo, G. Klein, J. P. Spatz, J. Cell Sci. 2012, 125, 3765
[163] P. Gilbert, K. Havenstrite, K. Magnusson, A. Sacco, N. Leonardi, P. Kraft, N. Nguyen, S. Thrun, M. Lutolf, H. Blau, Science 2010, 329, 1078.

[164] J. Liu, Y. Tan, H. Zhang, Y. Zhang, P. Xu, J. Chen, Y.-C. Poh, K. Tang, N. Wang, B. Huang, Nature Mater. 2012, 11, 734.

[165] C. Kiecker, C. Niehrs, Development. 2001, 128, 4189

[166] H. Kleinman, D. Philp, M. Hoffman, Curr. Opin. Biotech. 2003, 14, 526.

[167] C. S. Chen, M. Mrksich, S. Huang, G. M. Whitesides, D. E. Ingber, Science 1997, 276, 1425

[168] J. C. Love, L. A. Estroff, J. K. Kriebel, R. G. Nuzzo, G. M. Whitesides, Chem. Rev. 2005, 105, 1103.

[169] S. Coyer, A. García, E. Delamarche, Angew. Chem. Int. Edit. 2007, 46,6837

[170] R. Desai, M. Khan, S. Gopal, C. Chen, Integr. Biol. 2011, 3, 560.

[171] X. Tang, M. Ali, M. Saif, Soft Matter. 2012, 8, 7197.

[172] Q. Tseng, I. Wang, E. Duchemin-Pelletier, A. Azioune, N. Carpi, J. Gao, O. Filhol, M. Piel, M. Théry, M. Balland, Lab Chip. 2011, 11, 2231.

[173] A. Folch, B. H. Jo, O. Hurtado, D. J. Beebe, M. Toner, J. Biomed. Mater. Res. 2000, 52, 346.

[174] A. Azioune, M. Storch, M. Bornens, M. Théry, M. Piel, Lab Chip. 2009, 9, 1640.

[175] T. Vignaud, R. Galland, Q. Tseng, L. Blanchoin, J. Colombelli, M. Théry, J. Cell Sci. 2012, 125, 2134.

[176] J. Fink, M. Thery, A. Azioune, R. Dupont, F. Chatelain, M. Bornens, M. Piel, Lab Chip. 2007, 7, 672.

[177] R. D. Piner, J. Zhu, F. Xu, S. H. Hong, C. A. Mirkin, Science 1999, 283, 661

[178] K. Salaita, Y. Wang, C. Mirkin, Nature Nanotech. 2007, 2, 145.

[179] F. Huo, Z. Zheng, G. Zheng, L. Giam, H. Zhang, C. Mirkin, Science 2008, 321, 1658.

[180] Z. Zheng, W. Daniel, L. Giam, F. Huo, A. Senesi, G. Zheng, C. Mirkin, Angew. Chem. Int. Edit. 2009, 48, 7626.

[181] J. Chai, L. Wong, L. Giam, C. Mirkin, Proc. Natl. Acad. Sci. U.S.A. 2011, 108, 19521.

[182] S. Allazetta, S. Cosson, M. Lutolf, Chem. Commun. 2011, 47, 191.

[183] D. Chiu, N. Jeon, S. Huang, R. Kane, C. Wargo, I. Choi, D. Ingber, G. Whitesides, Proc. Natl. Acad. Sci. U.S.A. 2000, 97, 2408

[184] D. Anderson, S. Levenberg, R. Langer, Nat. Biotechnol. 2004, 22, 863

[185] C. Flaim, S. Chien, S. Bhatia, Nat. Methods. 2005, 2, 119.

[186] J. U. Park, M. Hardy, S. J. Kang, K. Barton, K. Adair, D. K. Mukhopadhyay, C. Y. Lee, M. S. Strano, A. G. Alleyne, J. G. Georgiadis, P. M. Ferreira, J. A. Rogers, Nature Mater. 2007, 6, 782

[187] J. U. Park, J. H. Lee, U. Paik, Y. Lu, J. A. Rogers, Nano Lett. 2008, 8, 4210.

[188] M. Onses, C. Song, L. Williamson, E. Sutanto, P. Ferreira, A. Alleyne, P. Nealey, H. Ahn, J. Rogers, Nature Nanotech. 2013, 8, 667.

[189] R. Glass, M. Möller, J. Spatz, Nanotechnology. 2003, 14, 1153.

[190] P. S. Joachim, M. Stefan, H. Christoph, M. Martin, H. Thomas, K. Michael, B. Hans-Gerd, Z. Paul, K. Bernd, Langmuir 2000, 16, 407.

[191] P. George, M. Doran, T. Croll, T. Munro, J. Cooper-White, Biomaterials 2009, 30, 4732.

[192] L. K. Kato, G. Nalini, D. D. Michael, A. L. Nathaniel, J. Hyunjung, T. Helen, B. Joona, M. C. Luis, ACS Macro Lett. 2012, 1, 758.

[193] W.-S. Yeo, M. Yousaf, M. Mrksich, J. Am. Chem. Soc. 2003, 125, 14944.

[194] W.-S. Yeo, M. Mrksich, Langmuir 2006, 22, 10816.

[195] M. Yousaf, B. Houseman, M. Mrksich, Angew. Chem. 2001, 113, 1127.

[196] M. Yousaf, B. Houseman, M. Mrksich, Proc. Natl. Acad. Sci. U.S.A. 2001, 98, 5992 
[197] D. Wright, B. Rajalingam, S. Selvarasah, M. Dokmeci, A. Khademhosseini, Lab Chip. 2007, 7, 1272.

[198] X. Jiang, R. Ferrigno, M. Mrksich, G. Whitesides, J. Am. Chem. Soc. 2003, 125, 2366

[199] X. Jiang, D. Bruzewicz, A. Wong, M. Piel, G. Whitesides, Proc. Natl. Acad. Sci. U.S.A. 2005, 102, 975.

[200] Y. Kikuchi, J. Nakanishi, H. Nakayama, T. Shimizu, Y. Yoshino, K. Yamaguchi, Y. Yoshida, Y. Horiike, Chem. Lett. 2008, 37, 1062.

[201] Y. Kikuchi, J. Nakanishi, T. Shimizu, H. Nakayama, S. Inoue, K. Yamaguchi, H. Iwai, Y. Yoshida, Y. Horiike, T. Takarada, M. Maeda, Langmuir 2008, 24, 13084.

[202] J. Nakanishi, Y. Kikuchi, T. Takarada, H. Nakayama, K. Yamaguchi, M. Maeda, Anal. Chim. Acta. 2006, 578, 100

[203] E. Hui, S. Bhatia, Proc. Natl. Acad. Sci. U.S.A. 2007, 104, 5722.

[204] S. van Dongen, P. Maiuri, E. Marie, C. Tribet, M. Piel, Adv. Mater. 2013, 25, 1687.

[205] M. S. Hahn, J. S. Miller, J. L. West, Adv. Mater. 2006, 18, 2679.

[206] S.-H. Lee, J. Moon, J. West, Biomaterials 2008, 29, 2962.

[207] R. Wylie, S. Ahsan, Y. Aizawa, K. Maxwell, C. Morshead, M. Shoichet, Nature Mater. 2011, 10, 799.

[208] J. Culver, J. Hoffmann, R. Poché, J. Slater, J. West, M. Dickinson, Adv. Mater. 2012, 24, 2344.

[209] K. Bhadriraju, M. Yang, S. Alom Ruiz, D. Pirone, J. Tan, C. Chen, Exp. Cell Res. 2007, 313, 3616

[210] S. Ruiz, C. Chen, Stem. Cells. 2008, 26, 2921.

[211] K. Kilian, B. Bugarija, B. Lahn, M. Mrksich, Proc. Natl. Acad. Sci. U.S.A. 2010, 107, 4872.

[212] M. Arnold, E. Cavalcanti-Adam, R. Glass, J. Blümmel, W. Eck, M. Kantlehner, H. Kessler, J. Spatz, ChemPhysChem. 2004, 5, 383.

[213] E. Cavalcanti-Adam, T. Volberg, A. Micoulet, H. Kessler, B. Geiger J. Spatz, Biophys. J. 2007, 92, 2964.

[214] C. Selhuber-Unkel, M. López-García, H. Kessler, J. Spatz, Biophys. J. 2008, 95, 5424.

[215] M. Schvartzman, M. Palma, J. Sable, J. Abramson, X. Hu, M. Sheetz, S. Wind, Nano Lett. 2011, 11, 1306.

[216] S. Coyer, A. Singh, D. Dumbauld, D. Calderwood, S. Craig, E. Delamarche, A. García, J. Cell Sci. 2012, 125, 5110.

[217] M. Thery, V. Racine, A. Pepin, M. Piel, Y. Chen, J. B. Sibarita, M. Bornens, Nat. Cell Biol. 2005, 7, 947.

[218] M. Thery, V. Racine, M. Piel, A. Pepin, A. Dimitrov, Y. Chen, J. B. Sibarita, M. Bornens, Proc. Natl. Acad. Sci. U.S.A. 2006, 103, 19771.

[219] M. Thery, A. Pepin, E. Dressaire, Y. Chen, M. Bornens, Cell Motil. Cytoskel. 2006, 63, 341.

[220] M. Thery, A. Jimenez-Dalmaroni, V. Racine, M. Bornens, F. Julicher Nature. 2007, 447, 493.

[221] Q. Tseng, E. Duchemin-Pelletier, A. Deshiere, M. Balland H. Guillou, O. Filhol, M. Théry, Proc. Natl. Acad. Sci. U.S.A. 2012 , 109, 1506

[222] H. Zhang, F. Landmann, H. Zahreddine, D. Rodriguez, M. Koch, M. Labouesse, Nature. 2011, 471, 99.

[223] W. Legant, J. Miller, B. Blakely, D. Cohen, G. Genin, C. Chen, Nat. Methods. 2010, 7, 969.

[224] C. Kraning-Rush, J. Califano, C. Reinhart-King, PLoS One. 2012, 7, e32572.

[225] N. Balaban, U. Schwarz, D. Riveline, P. Goichberg, G. Tzur I. Sabanay, D. Mahalu, S. Safran, A. Bershadsky, L. Addadi, B. Geiger, Nat. Cell Biol. 2001, 3, 466.

[226] P. W. Oakes, Y. Beckham, J. Stricker, M. L. Gardel, J. Cell Biol. 2012, 196, 363.

[227] Z. Liu, J. Tan, D. Cohen, M. Yang, N. Sniadecki, S. Ruiz, C. Nelson, C. Chen, Proc. Natl. Acad. Sci. U.S.A. 2010, 107, 9944

[228] V. Maruthamuthu, B. Sabass, U. Schwarz, M. Gardel, Proc. Natl. Acad. Sci. U.S.A. 2011, 108, 4708.

[229] T. Kobayashi, M. Sokabe, Curr. Opin. Cell Biol. 2010, 22, 669.
[230] M. Dembo, Y. Wang, Biophys. J. 1999, 76, 2307.

[231] U. S. Schwarz, N. Q. Balaban, D. Riveline, A. Bershadsky, B. Geiger, S. A. Safran, Biophys. J. 2002, 83, 1380

[232] B. Sabass, M. Gardel, C. Waterman, U. Schwarz, Biophys. J. 2008, 94, 207.

[233] J. Stricker, B. Sabass, U. S. Schwarz, M. L. Gardel, J. Phys.: Condens. Mat. 2010, 22, 194104.

[234] S. Plotnikov, A. Pasapera, B. Sabass, C. Waterman, Cell 2012, 151, 1513.

[235] C. Franck, S. Maskarinec, D. Tirrell, G. Ravichandran, PLoS One. 2011, 6, e17833.

[236] J. del Alamo, R. Meili, B. Alonso-Latorre, J. Rodríguez-Rodríguez, A. Aliseda, R. Firtel, J. Lasheras, Proc. Natl. Acad. Sci. U.S.A. 2007, 104, 13343.

[237] W. Legant, C. Choi, J. Miller, L. Shao, L. Gao, E. Betzig, C. Chen, Proc. Natl. Acad. Sci. U.S.A. 2013, 110, 881.

[238] H. Delanoë-Ayari, J. Rieu, M. Sano, Phys. Rev. Lett. 2010, 105, 248103.

[239] J. del Alamo, R. Meili, B. Alonso-Latorre, J. Rodríguez-Rodríguez, A. Aliseda, R. Firtel, J. Lasheras, Proc. Natl. Acad. Sci. U.S.A. 2007 104, 13343.

[240] N. Gjorevski, C. M. Nelson, Biophys. J. 2012, 103, 152.

[241] M. Hall, R. Long, X. Feng, Y. Huang, C.-Y. Hui, M. Wu, Exp. Cell Res. 2013, 319, 2396.

[242] T. Xavier, R. W. Michael, E. A. Thomas, M. Emil, A. W. David, P. B. James, J. F. Jeffrey, Nature Phys. 2009, 5,

[243] D. Tambe, C. Hardin, T. Angelini, K. Rajendran, C. Park, X. Serra-Picamal, E. Zhou, M. Zaman, J. Butler, D. Weitz, J. Fredberg, X. Trepat, Nature Mater. 2011, 10, 469.

[244] Y. T. Shiu, S. Li, W. A. Marganski, S. Usami, M. A. Schwartz, Y. L. Wang, M. Dembo, S. Chien, Biophys. J. 2004, 86, 2558

[245] S. Hur, J. del Álamo, J. Park, Y.-S. Li, H. Nguyen, D. Teng, K.-C. Wang, L. Flores, B. Alonso-Latorre, J. Lasheras, S. Chien, Proc. Natl. Acad. Sci. U.S.A. 2012, 109, 11110

[246] R. Meili, B. Alonso-Latorre, J. del Alamo, R. Firtel, J. Lasheras, Mol. Biol. Cell. 2010, 21, 405

[247] N. Gavara, P. Roca-Cusachs, R. Sunyer, R. Farré, D. Navajas, Biophys. J. 2008, 95, 464

[248] C. Galbraith, M. Sheetz, Proc. Natl. Acad. Sci. U.S.A. 1997, 94 9114

[249] C. Nelson, R. Jean, J. Tan, W. Liu, N. Sniadecki, A. Spector, C. Chen, Proc. Natl. Acad. Sci. U.S.A. 2005, 102, 11594.

[250] W. Legant, A. Pathak, M. Yang, V. Deshpande, R. McMeeking, C. Chen, Proc. Natl. Acad. Sci. U.S.A. 2009, 106, 10097.

[251] W. Legant, C. Chen, V. Vogel, Integr. Biol. 2012, 4, 1164.

[252] M. Sakar, D. Neal, T. Boudou, M. Borochin, Y. Li, R. Weiss, R. Kamm, C. Chen, H. Asada, Lab Chip. 2012, 12, 4976.

[253] R. Lam, Y. Sun, W. Chen, J. Fu, Lab Chip. 2012, 12, 1865.

[254] B. Ricart, M. Yang, C. Hunter, C. Chen, D. Hammer, Biophys. J. 2011, 101, 2620

[255] J. Mann, R. Lam, S. Weng, Y. Sun, J. Fu, Lab Chip. 2012, 12, 731.

[256] R. Lam, S. Weng, W. Lu, J. Fu, Integr. Biol. 2012, 4, 1289.

[257] N. Wang, J. D. Tytell, D. E. Ingber, Nat. Rev. Mol. Cell Biol. 2009, 10, 75.

[258] F. Chowdhury, S. Na, D. Li, Y.-C. Poh, T. Tanaka, F. Wang, N. Wang, Nature Mater. 2010, 9, 82

[259] M. P. Sheetz, Nat. Rev. Mol. Cell Biol. 2001, 2, 392.

[260] S. Siechen, S. Y. Yang, A. Chiba, T. Saif, Proc. Natl. Acad. Sci. U.S.A. 2009, 106, 12611

[261] G. T. Charras, M. A. Horton, Biophys. J. 2002, 82, 2970.

[262] N. Sniadecki, A. Anguelouch, M. Yang, C. Lamb, Z. Liu, S. Kirschner, Y. Liu, D. Reich, C. Chen, Proc. Natl. Acad. Sci. U.S.A. 2007, 104, 14553 
[263] D. Riveline, E. Zamir, N. Q. Balaban, U. S. Schwarz, T. Ishizaki, S. Narumiya, Z. Kam, B. Geiger, A. D. Bershadsky, J. Cell Biol. 2001, $153,1175$.

[264] R. Zhao, T. Boudou, W.-G. Wang, C. Chen, D. Reich, Adv. Mater. 2013, 25, 1699 .

[265] Y. Wang, N. Wang, Integr. Biol. 2009, 1, 565.

[266] C. Grashoff, B. Hoffman, M. Brenner, R. Zhou, M. Parsons, M. Yang, M. McLean, S. Sligar, C. Chen, T. Ha, M. Schwartz, Nature. 2010, 466, 263.

[267] S. Iwai, T. Uyeda, Proc. Natl. Acad. Sci. U.S.A. 2008, 105, 16882.

[268] Q. le Duc, Q. Shi, I. Blonk, A. Sonnenberg, N. Wang, D. Leckband, J. de Rooij, J. Cell Biol. 2010, 189, 1107.

[269] F. Margadant, L. L. Chew, X. Hu, H. Yu, N. Bate, X. Zhang, M. Sheetz, PLoS Biol. 2011, 9, e1001223.

[270] G. Baneyx, L. Baugh, V. Vogel, Proc. Natl. Acad. Sci. U.S.A. 2001, 98, 14464.

[271] E. Klotzsch, M. Smith, K. Kubow, S. Muntwyler, W. Little, F. Beyeler, D. Gourdon, B. Nelson, V. Vogel, Proc. Natl. Acad. Sci. U.S.A. 2009, 106, 18267.

[272] M. Smith, D. Gourdon, W. Little, K. Kubow, R. Eguiluz, S. Luna-Morris, V. Vogel, PLoS Biol. 2007, 5, e268.

[273] D. Stabley, C. Jurchenko, S. Marshall, K. Salaita, Nat. Methods. 2012, 9, 64 .

[274] M. Morimatsu, A. Mekhdjian, A. Adhikari, A. Dunn, Nano Lett. 2013, In Press.

[275] Y. Liu, K. Yehl, Y. Narui, K. Salaita, J. Am. Chem. Soc. 2013, 135, 5320.

[276] X. F. Wang, T. Ha, Science 2013, 340, 991.

[277] B. Baker, C. Chen, J. Cell Sci. 2012, 125, 3015

[278] M. Ochsner, M. Textor, V. Vogel, M. Smith, PLoS One. 2010, 5, e9445.

[279] K. R. King, C. C. J. Wang, M. R. Kaazempur-Mofrad, J. P. Vacanti, I. T. Borenstein, Adv. Mater. 2004, 16, 2007.

[280] M. D. Tang, A. P. Golden, J. Tien, Adv. Mater. 2004, 16, 1345.

[281] M. Cabodi, N. Choi, J. Gleghorn, C. Lee, L. Bonassar, A. Stroock, J. Am. Chem. Soc. 2005, 127, 13788.

[282] M. Tang, A. Golden, J. Tien, J. Am. Chem. Soc. 2003, 125, 12988

[283] A. Golden, J. Tien, Lab Chip. 2007, 7, 720.

[284] J. Park, B. Chung, W. Lee, J. Kim, M. Brigham, J. Shim, S. Lee, C. Hwang, N. Durmus, U. Demirci, A. Khademhosseini, Biotechnol. Bioeng. 2010, 106, 138.

[285] Y. Zheng, J. Chen, M. Craven, N. Choi, S. Totorica, A. Diaz-Santana, P. Kermani, B. Hempstead, C. Fischbach-Teschl, J. López, A. Stroock, Proc. Natl. Acad. Sci. U.S.A. 2012, 109, 9342.

[286] D.-H. T. Nguyen, S. Stapleton, M. Yang, S. Cha, C. Choi, P. Galie, C. Chen, Proc. Natl. Acad. Sci. U.S.A. 2013, 110, 6712.

[287] J. W. Nichol, S. T. Koshy, H. Bae, C. M. Hwang, S. Yamanlar, A. Khademhosseini, Biomaterials 2010, 31, 5536.

[288] J. He, M. Mao, Y. Liu, J. Shao, Z. Jin, D. Li, Adv. Healthcare Mater. 2013, In Press.

[289] G. Price, K. Chu, J. Truslow, M. Tang-Schomer, A. Golden, J. Mertz, J. Tien, J. Am. Chem. Soc. 2008, 130, 6664.

[290] N. Choi, M. Cabodi, B. Held, J. Gleghorn, L. Bonassar, A. Stroock, Nature Mater. 2007, 6, 908.

[291] Y. Ling, J. Rubin, Y. Deng, C. Huang, U. Demirci, J. M. Karp, A. Khademhosseini, Lab Chip. 2007, 7, 756.

[292] J. Morgan, P. Delnero, Y. Zheng, S. Verbridge, J. Chen, M. Craven, N. Choi, A. Diaz-Santana, P. Kermani, B. Hempstead, J. López, T. Corso, C. Fischbach, A. Stroock, Nat. Protoc. 2013, 8, 1820.

[293] J. Baranski, R. Chaturvedi, K. Stevens, J. Eyckmans, B. Carvalho, R. Solorzano, M. Yang, J. Miller, S. Bhatia, C. Chen, Proc. Natl. Acad. Sci. U.S.A. 2013, In Press.

[294] L. M. Bellan, M. Pearsall, D. M. Cropek, R. Langer, Adv. Mater. 2012, 24, 5187
[295] J. Miller, K. Stevens, M. Yang, B. Baker, D.-H. T. Nguyen, D. Cohen, E. Toro, A. Chen, P. Galie, X. Yu, R. Chaturvedi, S. Bhatia, C. Chen, Nature Mater. 2012, 11, 768

[296] D. Therriault, S. White, J. Lewis, Nature Mater. 2003, 2, 265.

[297] W. Wu, A. DeConinck, J. Lewis, Adv. Mater. 2011, 23, H178.

[298] J. H. Huang, J. Kim, N. Agrawal, A. P. Sudarson, J. E. Maxim, A. Jayaraman, V. M. Ugaz, Adv. Mater. 2009, 21, 3567.

[299] W. Tan, T. Desai, Biomaterials 2004, 25, 1355.

[300] V. L. Tsang, A. A. Chen, L. M. Cho, K. D. Jadin, R. L. Sah, S. DeLong, J. L. West, S. N. Bhatia, FASEB J. 2007, 21, 790.

[301] D. Albrecht, G. Underhill, T. Wassermann, R. Sah, S. Bhatia, Nat. Methods. 2006, 3, 369.

[302] Y. S. Hwang, B. G. Chung, D. Ortmann, N. Hattori, H. C. Moeller, A. Khademhosseini, Proc. Natl. Acad. Sci. U.S.A. 2009, 106, 16978.

[303] C. Nelson, M. Vanduijn, J. Inman, D. Fletcher, M. Bissell, Science 2006, 314, 298

[304] Y. A. Du, E. Lo, S. Ali, A. Khademhosseini, Proc. Natl. Acad. Sci. U.S.A. 2008, 105, 9522.

[305] G. Eng, B. Lee, H. Parsa, C. Chin, J. Schneider, G. Linkov, S. Sia, G. Vunjak-Novakovic, Proc. Natl. Acad. Sci. U.S.A. 2013, 110, 4551.

[306] D. Huh, B. Matthews, A. Mammoto, M. Montoya-Zavala, H. Hsin, D. Ingber, Science 2010, 328, 1662.

[307] D. Huh, D. Leslie, B. Matthews, J. Fraser, S. Jurek, G. Hamilton, K. Thorneloe, M. McAlexander, D. Ingber, Sci. Transl. Med. 2012, 4, 159 ra147.

[308] H. Kim, D. Huh, G. Hamilton, D. Ingber, Lab Chip. 2012, 12, 2165

[309] K.-J. Jang, K.-Y. Suh, Lab Chip. 2010, 10, 36

[310] K.-J. Jang, H. Cho, D. H. Kang, W. Bae, T.-H. Kwon, K.-Y. Suh, Integr. Biol. 2011, 3, 134.

[311] K.-J. Jang, A. Mehr, G. Hamilton, L. McPartlin, S. Chung, K.-Y. Suh, D. Ingber, Integr. Biol. 2013, In Press.

[312] C. Puleo, W. Mclntosh Ambrose, T. Takezawa, J. Elisseeff, T.-H. Wang, Lab Chip. 2009, 9,

[313] A. Grosberg, P. Alford, M. McCain, K. Parker, Lab Chip. 2011, 11, 4165.

[314] J.-M. Peyrin, B. Deleglise, L. Saias, M. Vignes, P. Gougis, S. Magnifico, S. Betuing, M. Pietri, J. Caboche, P. Vanhoutte, J.-L. Viovy, B. Brugg, Lab Chip. 2011, 11, 3663.

[315] Y. Nakao, H. Kimura, Y. Sakai, T. Fujii, Biomicrofluidics. 2011, 5, 022212.

[316] E. Cimetta, C. Cannizzaro, R. James, T. Biechele, R. Moon, N. Elvassore, G. Vunjak-Novakovic, Lab Chip. 2010, 10, 3277.

[317] J. Diao, L. Young, S. Kim, E. Fogarty, S. Heilman, P. Zhou, M. Shuler, M. Wu, M. DeLisa, Lab Chip. 2006, 6, 381.

[318] J. Sung, M. Shuler, Lab Chip. 2009, 9, 1385.

[319] J. Sung, C. Kam, M. Shuler, Lab Chip. 2010, 10, 446.

[320] M. Esch, T. King, M. Shuler, Annu. Rev. Biomed. Eng. 2011, 13, 55.

[321] Y. Imura, K. Sato, E. Yoshimura, Anal. Chem. 2010, 82, 9983.

[322] E. Kang, J. Ryoo, G. Jeong, Y. Choi, S. Jeong, J. Ju, S. Chung, S. Takayama, S.-H. Lee, Adv. Mater. 2013, 25, 2167.

[323] J. P. Thiery, Nat. Rev. Cancer. 2002, 2, 442.

[324] P. Friedl, D. Gilmour, Nat. Rev. Mol. Cell Biol. 2009, 10, 445.

[325] M. Swartz, M. Fleury, Annu. Rev. Biomed. Eng. 2007, 9, 229.

[326] J. Shields, M. Fleury, C. Yong, A. Tomei, G. Randolph, M. Swartz, Cancer Cell. 2007, 11, 526.

[327] J. Munson, R. Bellamkonda, M. Swartz, Cancer Res. 2013, 73, 1536.

[328] S. Giampieri, C. Manning, S. Hooper, L. Jones, C. Hill, E. Sahai, Nat. Cell Biol. 2009, 11, 1287.

[329] E. Roussos, J. Condeelis, A. Patsialou, Nat. Rev. Cancer. 2011, 11, 573

[330] W. J. Polacheck, I. K. Zervantonakis, R. D. Kamm, Cell Mol. Life Sci. 2013, 70, 1335

[331] K. Kessenbrock, V. Plaks, Z. Werb, Cell 2010, 141, 52.

[332] R. Kalluri, M. Zeisberg, Nat. Rev. Cancer. 2006, 6, 392. 
[333] K. Wolf, Y. I. Wu, Y. Liu, J. Geiger, E. Tam, C. Overall, M. S. Stack, P. Friedl, Nat. Cell Biol. 2007, 9, 893.

[334] J. Wyckoff, W. G. Wang, E. Y. Lin, Y. R. Wang, F. Pixley, E. R. Stanley, T. Graf, J. W. Pollard, J. Segall, J. Condeelis, Cancer Res. 2004, 64, 7022.

[335] J. Condeelis, J. W. Pollard, Cell 2006, 124, 263.

[336] J. Wyckoff, Y. Wang, E. Lin, J.-f. Li, S. Goswami, E. Stanley, J. Segall, J. Pollard, J. Condeelis, Cancer Res. 2007, 67, 2649.

[337] B. Z. Qian, Y. Deng, J. H. Im, R. J. Muschel, Y. Y. Zou, J. F. Li, R. A. Lang, J. W. Pollard, PLoS One. 2009, 4, e6562.

[338] M. J. Pittet, R. Weissleder, Cell 2011, 147, 983.

[339] E. Beerling, L. Ritsma, N. Vrisekoop, P. W. B. Derksen, J. van Rheenen, J. Cell Sci. 2011, 124, 299.

[340] J. Song, S. Cavnar, A. Walker, K. Luker, M. Gupta, Y.-C. Tung, G. Luker, S. Takayama, PLoS One. 2009, 4, e5756.

[341] S. Chung, R. Sudo, V. Vickerman, I. Zervantonakis, R. Kamm, Ann. Biomed. Eng. 2010, 38, 1164.
[342] D. J. Beebe, J. S. Moore, J. M. Bauer, Q. Yu, R. H. Liu, C. Devadoss, B. H. Jo, Nature. 2000, 404, 588

[343] K. Sung, N. Yang, C. Pehlke, P. Keely, K. Eliceiri, A. Friedl, D. Beebe, Integr. Biol. 2011, 3, 439.

[344] I. Zervantonakis, S. Hughes-Alford, J. Charest, J. Condeelis, F. Gertler, R. Kamm, Proc. Natl. Acad. Sci. U.S.A. 2012, 109, 13515.

[345] W. Polacheck, J. Charest, R. Kamm, Proc. Natl. Acad. Sci. U.S.A. 2011, 108, 11115.

[346] T. Hagemann, S. C. Robinson, M. Schulz, L. Trumper, F. R. Balkwill, C. Binder, Carcinogenesis. 2004, 25, 1543

[347] M. Chen, J. Whisler, J. Jeon, R. Kamm, Integr. Biol. 2013, 5, 1262.

[348] M. L. Moya, Y. H. Hsu, A. P. Lee, C. C. W. Hughes, S. C. George, Tissue Eng. Part C Methods 2013, 19, 730.

[349] K. Konstantopoulos, S. N. Thomas, Annu. Rev. Biomed. Eng. 2009, $11,177$.

[350] C. Drifka, K. Eliceiri, S. Weber, W. Kao, Lab Chip. 2013, 13, 3965. 